\title{
Summaries of plenary, symposia, and oral sessions at the XXII World Congress of Psychiatric Genetics, Copenhagen, Denmark, 12-16 October 2014
}

\author{
Monica Aas ${ }^{a}{ }^{*}$, Gabriëlla A.M. Blokland ${ }^{b, c}$, Samuel J.R.A. Chawner ${ }^{\mathrm{h}}$, Shing-Wan Choi ${ }^{\mathrm{n}}$, Jose \\ Estrada $^{\mathrm{e}}$, Annika Forsingdal $^{\circ}$, Maximilian Friedrich ${ }^{p}$, Suhas Ganesham ${ }^{r}$, Lynsey Hall ${ }^{i}$, \\ Denise Haslinger $q$, Laura Huckins ${ }^{j}$, Erik Loken ${ }^{\dagger}$, Stefanie Malan-Müller $^{\mathrm{s}}$, Joanna Martin ${ }^{\mathrm{h}}$, \\ Zuzanna Misiewicz ${ }^{t}$, Luca Pagliaroliu, Antonio F. Pardiñas ${ }^{h}$, Claudia Pisanuv ${ }^{v}$, Giorgia

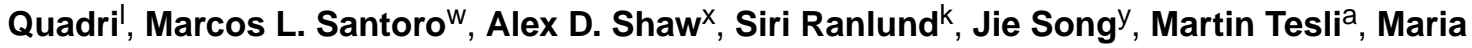 \\ Tropeano $^{\mathrm{m}}$, Monique van $\operatorname{der}_{\text {Voet }^{z}}$, Kate Wolfe ${ }^{l}$, Freida K. Cormack ${ }^{\mathrm{g}, \dagger}$, and Lynn DeLisi ${ }^{\mathrm{d}, \neq}$
}

aNORMENT, Oslo University Hospital, University of Oslo, Oslo, Norway b Psychiatric and Neurodevelopmental Genetics Unit, Department of Psychiatry/Center for Human Genetic Research, Massachusetts General Hospital, Harvard Medical School, Boston 'Stanley Center for Psychiatric Research, Broad institute of MIT and Harvard, Cambridge dHarvard Medical School, Brockton VA boston Healthcare System, Brockton, Massachusetts ${ }^{\mathrm{C} C e n t e r}$ of Excellence in Neuroscience, Texas Tech University Health Sciences Center, El Paso, Texas ${ }^{f}$ Virginia Institute for Psychiatric and Behavioral Genetics, Richmond, Virginia IInstitute of Psychiatric Research, Indianapolis, Indiana, USA hMRC Centre for Neuropsychiatric Genetics and Genomics, Institute of Psychological Medicine and Clinical Neurosciences, Cardiff University, Cardiff iDivision of Psychiatry, School of Clinical Sciences, Royal Edinburgh Hospital, The University of Edinburgh, Edinburgh jWellcome Trust Sanger Institute, Hinxton kDivision of Psychiatry, University College London 'Molecular Psychiatry Laboratory, Division of Psychiatry, University College London mMRC Social, Genetic and Developmental Psychiatry Centre, Institute of Psychiatry, King's College London, London, UK nDepartment of Psychiatry, The University of Hong Kong, Hong Kong ${ }^{\circ} \mathrm{H}$. Lundbeck A/S, Department of Synaptic Transmission, In Vitro, Valby, Denmark PDepartment of Psychiatry, Division of Molecular Psychiatry, University of Wuerzburg, Wuerzburg aDepartment of Child and Adolescent Psychiatry, Psychosomatics and Psychotherapy, JW Goethe University, Frankfurt am Main, Germany 'National Institute of Mental Health and NeuroSciences, Bangalore, India 'Department of Psychiatry, Division of Molecular Biology and Human Genetics, Faculty of Medicine and Health Sciences, SA MRC Centre for TB Research, DST/NRF Centre of Excellence for Biomedical Tuberculosis Research, Stellenbosch University, Cape Town, South Africa tDepartment of Biosciences, Faculty of Biological and Environmental Sciences, University of Helsinki, Helsinki, Finland unstitute of Medical Chemistry, Molecular

Correspondence to Freida K. Cormack, BA, Institute of Psychiatric Research, Neuroscience Research Building, 320 W. 15th Street, Indianapolis, IN 46202, USA, Tel: + 1317274 8382; fax: + 1317231 0201; fcormack@iupui.edu.

*Rapporteurs in alphabetical order, all performed equal work.

†reida K. Cormack is the coordinator of rapporteurs and this summary.

tLynn DeLisi is the editor of reports.

Conflicts of interest

There are no conflicts of interest. 
Biology and Pathobiochemistry, Semmelweis University, Budapest, Hungary vDepartment of Biomedical Sciences, Section of Neuroscience and Clinical Pharmacology, University of Cagliari, Monserrato, Italy wDepartment of Morphology and Genetics, Federal University of Sao Paulo, Sao Paulo, Brazil ${ }^{\times}$Neuroscience Research Australia, Barker Street Randwick, Sydney, New South Wales, Australia yDepartment of Medical Epidemiology and Biostatistics, Karolinska Institute, Solna, Sweden zDepartment of Human Genetics, Donders Institute for Brain, Cognition and Behavior, Radboud University Medical Center, Nijmegen, The Netherlands

\section{Abstract}

The XXII World Congress of Psychiatric Genetics, sponsored by the International Society of Psychiatric Genetics, took place in Copenhagen, Denmark, on 12-16 October 2014. A total of 883 participants gathered to discuss the latest findings in the field. The following report was written by student and postdoctoral attendees. Each was assigned one or more sessions as a rapporteur. This manuscript represents topics covered in most, but not all of the oral presentations during the conference, and contains some of the major notable new findings reported. Psychiatr Genet 26:147 Copyright @ 2016 Wolters Kluwer Health, Inc. All rights reserved.

\section{Keywords}

attention-deficit hyperactivity disorder; bipolar disorder; genetics; genomics; International Society of Psychiatric Genetics; mood disorder; schizophrenia; World Congress of Psychiatric Genetics

\section{Introduction}

The XXII World Congress of Psychiatric Genetics, sponsored by the International Society of Psychiatric Genetics (ISPG), took place on 12-16 October 2014, in Copenhagen, Denmark. A total of 883 researchers in the fields of psychiatry, psychology, and molecular genetics attended the conference to discuss the latest technological advances in the field and new findings on the genetics of mental disorders.

The ISPG was first established as a nonprofit corporation in the USA in 1992 and is now a worldwide organization that strives for the highest methodologies to the study of psychiatric disorders. It was formed to provide a stable structure for continual congresses in this field with the mission of overseeing an annual gathering at different international locations. The congress was co-chaired by Dr Ole Mors, Dr Thomas G. Schulze, and Dr Thomas Werge. Rapporteurs for these sessions were students or postdocs who covered the presentations. They were tasked to summarize individual sessions as well as relevant discussions. Similar accounts of the 2007, 2008, 2009, 2010, 2011, 2012, 2013, and 2014 congress held in New York (New York), Osaka (Japan), San Diego (California), Athens (Greece), Washington (District of Colombia), Hamburg (Germany), and Boston (Massachusetts), have been published previously (Alkelai et al., 2008; Bergen et al., 2009, 2011; Amstadter et al., 2010; Dai et al., 2012; Anderson-Schmidt et al., 2013, Akpudo et al., 2014). 
The following report represents the major findings covered during most oral sessions and they appear together under major topics, irrespective of the timing of specific sessions.

\section{Keynote lecture: optical deconstruction of fully assembled biological systems - speaker Dr Karl Deisseroth (reported by Annika Forsingdal)}

Dr Karl Deisseroth (Stanford University, USA) aims to achieve a deep understanding of basic biological mechanisms that can facilitate a better understanding of psychiatric diseases. To this end, and to achieve a deeper understanding of biological processes Deisseroth's lab have developed the technologies Optogenetics and CLARITY. Optogenetics can induce patterns of activity or inhibition by illumination, with spatial resolution in specific cell types (Deisseroth, 2011). The first optogenetic studies showed how opsins could drive action potentials and affect behavior in animals.

There are two main categories of opsins: ion channels and pumps. Despite thorough searches, no inhibitory channels have been found. In 2012, the crystal structure of channelrhodopsin was obtained (Kato et al., 2012). This enabled engineering of channelrhodopsin, ultimately transforming it into a chloride channel, the first inhibitory ion channel opsin (Wietek et al., 2014). Engineering was performed by introducing mutations that were considered to increase the specificity toward chloride. Channelrhodopsin mutants were then expressed in primary neurons that were patched to study their effect on the channel properties. The mutants that had increased specificity for chloride were combined into a chloride channel that expressed well. Another characteristic that was searched for in the opsins was a stable change (i.e. to keep the cell switched off by a single light beam). This was achieved by the development of SwiChRs (Berndt et al., 2014).

Spatial specificity can be achieved with adenoviral constructs using a cell type-specific promoter; light can either be delivered to the same location as the virus or to another location. This spatial specificity has been exploited to study the circuitries and projections underlying different behaviors. By driving the basolateral amygdala (BLA) and projections from the BLA to the central nucleus of the amygdala (CeA), it was shown that BLA-CeA increases anxiolytic behavior in mice (Tye et al., 2011). Further optogenetic studies of the circuitries involved in anxiety and anxiolytic behavior described a role for the bed nucleus of the stria terminalis in anxiety-related behavior. It was shown that some projections from the bed nucleus of the stria terminalis were anxiolytic, whereas others increased anxiety (Kim et al., 2013).

Optogenetic studies of projections had never been tested without stimulation. Fiber photometry was developed to do exactly this. The technique enables measurement of activity of specific neurons (Gunaydin et al., 2014). Previous studies had shown that social behavior can be turned up and down by turning on dopamine neurons in the ventral tegmental area (VTA) [tyrosine hydroxylase (TH)-positive neurons]. VTA-TH neurons in a mouse become active when the animal is exploring a novel mouse or a novel object. This phenomenon was further investigated by moving the recording fiber to the nucleus accumbens (NAc). In the NAc, there is a stronger signal from the projections for social novelty than for novel objects. It was also shown that driving projection from VTA to NAc increased sociability of the 
mice. Projections from VTA to the prefrontal cortex (PFC) do not increase sociability, but makes the animal more anxious, measured by less time spent in the open arms of an elevated plus maze (Gunaydin et al., 2014).

Dr Deisseroth's lab also developed a technique for imaging intact tissue (e.g. a whole brain). Intact tissue is transformed into a hydrogel-hybridized form, which allows removal of lipids. When the lipids are removed, the light-scattering effect of the tissue is reduced and the tissue effectively becomes transparent (Chung et al., 2013). The tissue can then be stained with cell type-specific markers to examine specific cell types. Future plans include combining clarity with activity imaging.

\section{Plenary sessions}

\section{Selective serotonin reuptake inhibitor pharmacogenomics and pharmacometabolomics - speaker: Dr Richard Weinshilboum (reported by Claudia Pisanu)}

Dr Richard Weinshilboum (Mayo Medical School, Mayo Clinic, USA) first emphasized the importance of international consortia and collaborations between groups with complementary and overlapping expertise. Pharmacogenomics and pharmacometabolomics are central aspects of personalized medicine, which recognizes as major clinical goals avoidance of adverse drug reactions, maximization of drug efficacy, and selection of responsive patients. The scientific goals of personalized medicine include linking genomic and metabolomic variation to phenotypic variation (to predict which patients are going to experience an adverse reaction and which patients are going to respond or not respond to a drug), determination of the mechanisms responsible for that link, and, eventually, translation of that link into a better diagnosis, treatment, and prevention of disease.

Pharmacogenetics has evolved into pharmacogenomics, a broader approach used to study variations across the whole genome.

Professor Weinshilboum described - as an example - how breast cancer treatment has changed profoundly across the years as we have been able to use drugs such as selective estrogen receptor (ER) modulators, aromatase inhibitors, and trastuzumab to subclassify the disease and individualize therapy, and explained that this is a path that we may be able to follow with psychiatric diseases. In this sense, recent studies have used selective serotonin reuptake inhibitors (SSRIs) as molecular probes for major depressive disorder (MDD) molecular mechanisms. Professor Weinshilboum remembered Julius Axelrod's major discovery of the reuptake of monoamine neurotransmitters, the mechanism inhibited by SSRI, and explained the design of the Mayo PGRN Citalopram-Escitalopram Clinical Trial, aimed at better understanding the SSRI molecular mechanism of action using both pharmacogenomics and pharmacometabolomics (Ji et al., 2013). The discovery study involved a large sample of $529 \mathrm{MDD}$ patients at the Mayo Clinic, whereas the replication study was carried out on the DNA of patients from the Sequenced Treatment Alternatives to Relieve Depression (STAR*D) study (Trivedi et al., 2006; Howland, 2008; Zisook et al., 2008) and samples from the International SSRI Pharmacogenomics Consortium (PGRN ISPC). In the Citalopram-Escitalopram Clinical Trial, most of the patients gained some benefit from SSRI therapy, but a number of patients still showed moderate to severe 
symptomatology. The rs11144870 single nucleotide polymorphism (SNP) located in an intron of the riboflavin kinase $(R F K)$ gene $\left(P=1.16 \times 10^{-6}\right)$ was associated with the response outcome. However, the association has not been replicated. It is well known that many genome-wide association study (GWAS) findings on SSRI response, like many GWAS in psychiatry, have proven difficult to replicate (Ising et al., 2009; Garriock et al., 2010; Uher et al., 2010; Ji et al., 2011) and included among possible explanations could be phenotypic heterogeneity and lack of objective biomarkers.

Genome-wide significant associations were observed for escitalopram concentrations with SNPs in or near the CYP2C19 gene (rs1074145, $P=4.1 \times 10^{-9}$ ) and with $S$ didesmethylcitalopram concentration for SNPs near the CYP2D6 locus (rs1065852, $P=2.0$ $\times 10^{-16}$ ) (Ji et al., 2014). Moreover, other hits with genome-wide significance were obtained after adjusting for the effect of $C Y P 2 C 19$ functional alleles. Plasma metabolomic assays using samples from the same patients were also performed using an LC-electrochemical array platform (Ji et al., 2014). This pharmacometabolomics-informed pharmacogenomic research strategy was applied to plasma samples from 20 escitalopram remitters and 20 nonremitter MDD patients, and showed that glycine levels were negatively associated with treatment outcome $(P=0.0054)$ (Ji et al., 2011). When genotyping tag SNPs of genes encoding glycine synthesis and degradation enzymes in 529 SSRI-treated MDD patients, the rs10975641 SNP in the glycine dehydrogenase gene was associated with treatment outcome (Ji et al., 2011). Moreover, the subsequent application of a pharmacometabolomics-informed pharmacogenomics research strategy involving 300 MDD patients identified a series of candidate genes that are being pursued using functional genomic techniques.

Finally, during the discussion, Professor Weinshilboum stressed how pharmacometabolomics is an informative tool when observations from plasma metabolites are linked to genetic variation.

\section{Dr Christine Klein (University of Lübeck, Germany) - translating genetic findings into clinical practice: the Parkinson example (reported by Zuzanna Misiewicz)}

Almost two decades after the first mutation responsible for Parkinson's disease (PD) was identified (Polymeropoulos et al., 1996), Dr Christine Klein (University of Lübeck, Germany) spoke on translation of genetic findings into pathways to therapy and prevention, as based on the Parkinson example. The content of her presentation reflected accurately the theme of the XXII World Congress of Psychiatric Genetics.

Dr Klein introduced the topic by briefly reviewing the genetic nomenclature of PD with a special focus on the monogenic, both recessive and dominant, forms of PDs caused by defects in single genes. They account for around $5 \%$ of all sporadic cases; therefore, although well established, they are relatively rare in comparison with idiopathic forms of likely multifactorial etiology. The idiopathic forms comprise the majority of Parkinson cases $(95 \%)$. They are based on a more complex background, where combined effects of genetic variants in multiple genes, the environment, the complex interaction between them as well as other risk factors contribute toward the pathogenesis (Klein and Westenberger, 2012). More recently, GWASs have enabled a more direct approach in genetics of PD. As Dr Klein highlighted later in her presentation, the five performed GWAS and the subsequent meta- 
analyses (International Parkinson Disease Genomics Consortium et al., 2011) constituted a major contribution in genetic research of PD and facilitated the identification of further genetic risk factors associated with idiopathic PD, such as $S N C A$ and $L R R K 2$ genes, reported previously in monogenic PD.

Subsequently, Dr Klein vividly described the most common forms of dominant parkinsonism with mutations in the $L R R K 2$ gene and the $S N C A$ gene, and recessive parkinsonism, with a mutation in the Parkin gene. She presented examples of clinical phenotypes representative for each of them, respectively, rigidity, rest tremor, and bradykinesia. She stressed that all of them are indistinguishable from idiopathic cases of PD. She further emphasized that both forms of PD tend to converge on common pathophysiological pathways, such as mitochondrial dysfunction (PINK1, Parkin, DJ-1), endosomal vesicle protein recycling ( $L R R K 2)$, and protein degradation (VPS35). Following Malkus et al. (2009), Dr Klein referred to it as a Bermuda Triangle.

All of the examples of similarities presented by Dr Klein allowed her to confidently conclude that the genetic PD can be used as an excellent model of PD. It allows us to more fully understand the complicated idiopathic form, a knowledge that can be applied in clinical practice and genetic consulting leading to earlier diagnosis of at-risk individuals.

Moreover, Dr Klein noted an interesting comorbidity between PD and psychiatric diseases, such as depression, anxiety, dementia, or sleep problems (Park and Stacy, 2009). For example, in idiopathic PD, about $30 \%$ of the patients suffer from depression, whereas the general prevalence in the population is about $15 \%$. Furthermore, $41 \%$ of the carriers of the PINK1 mutation experience anxiety, which is significantly higher than the frequency in the general population (15-25\%). These observations may underscore the fact that underlying biological processes among these distinct phenotypes are at least partially convergent.

Although a great deal of knowledge has been gained in the field of PD genetics, little progress has been made in translating genetic findings into clinical practice so far. The development of new disease models, such as animal models and induced pluripotent stem cells (iPSC), has contributed toward major advancements in the field. The examples presented by Dr Klein included a study by Vos et al. (2012), in which a high dosage of vitamin $\mathrm{K}_{2}$ could rescue pink1 mutation deficiency. The group led by Dr Klein currently tests this mitochondrial enhancer strategy on patients with the PINK1 mutation.

In conclusion, Dr Klein's presentation showed that the complex picture of idiopathic PD provides cues of possible pathways to therapy and prevention and serves as an example for psychiatric diseases.

\section{Dr Tine Bryan Stensbøl (Lundbeck), NEWMEDS - a public private partnership in psychiatry - objectives, outcomes, obstacles, and opportunities (reported by Annika Forsingdal)}

Tine Bryan Stensbøl (Lundbeck) described the NEWMEDS consortium as a new way of collaborating between academia and industry. The Innovative Medicines Initiative was created with the vision of enhancing the position of Europe in biomedical research. One of the objectives was to invest in precompetitive collaborations between industry and academic 
partners Dr Stensbøl invited the European pharmaceutical companies who were active in psychiatry in 2008 to Lundbeck. The companies agreed on four key deliverables that they expected from the collaboration: (i) identification of transcriptomic markers/blood markers relevant for segmentation and stratification of patient groups; (ii) development of preclinical models that translate systems biology of disease; (iii) development of animal models that translate to clinical endpoints; (iv) development of functional imaging and molecular neuroimaging paradigms for drug discovery in depression and schizophrenia (SCZ). In terms of intellectual property, Dr Stensbøl stated: 'what we were not willing to share 5 years ago we are definitely willing to share today'. Academic research groups could apply to become part of the consortium and several academic groups were selected led by Shitij Kapur at Kings College London. Thereby, the NEWMEDS consortium was born under the Innovative Medicines Initiative umbrella in 2009. The partners of the NEWMEDS consortium agreed that the three major bottlenecks in the field were as follows:(i) lack of relevant disease models; (ii) lack of tools and tests in healthy volunteers to provide early indications of efficacy; (iii) the reliance of clinical trials on Diagnostic and statistical manual of mental disorders, 4th ed. (DSM-IV) and the heterogeneous groups of patients this resulted in. To address these challenges in the field, 11 work packages were outlined. Each work package was assigned an industry and an academic lead, which proved to be a good strategy because it forced the industry partners to take part in leading the work.

Initially, 14 animal models of SCZ were run through proteomic markers, but this approach was abandoned quickly. However, a smaller private-public partnership, of which Lundbeck was a part of, had generated three copy number variant (CNV) mouse models and these were shared within the NEWMEDS consortium.

Three successful outcomes of NEWMEDS that one partner could not have obtained on their own are (i) the clinical trial database with data from 29 placebo-controlled SCZ trials with 23000 patients. Evaluation of these data showed that data can be analyzed already after 4 weeks instead of 6 weeks and that the placebo effect is lower in women than in men. (ii) Testing of cognition battery across species and sites. (iii) Advanced understanding of CNV biology partly through cognitive tests of deCODE CNV patients.

The CNV mice will be commercially available through a vendor.

\section{Dr Oluf Borbye Pedersen (University of Copenhagen) - the gut microbiota and the impact on human health (reported by Laura Huckins)}

Dr Pedersen stated that we might think of humans as 'supra-organisms', with two major genomes: the human genome and the microbiome (defined as the collective genomics of bacteria). Unlike the human genome, the structural configuration of the microbiome changes often. The largest reservoir of bacteria in humans is the gut, containing roughly $1-2 \mathrm{~kg}$. Gut bacteria may be subdivided into six major phyla, and serve a variety of functions, including metabolic function, modulation of gut nutrient sensing, and conferring gut immunity.

The microbiome plays a role early in life as microbial components pass to the fetus through the placenta and amniotic fluid. During natural vaginal birth, the baby is further colonized with bacteria, for example lactobacillus, from the mother's vagina and feces, a process that 
is vital for normal immune system development. Microbiotic interaction between mother and child continues beyond birth, as breast milk increases DNA methylation and gene transcription rates, and has long-term effects on metabolic target genes.

The microbiome seems to play a role in psychiatric symptoms and disorders, and may act on a microbiome-gut-brain axis of communication, regulating anxiety, mood, cognition, and pain (Montiel-Castro et al., 2013). Further, there is epidemiological evidence suggesting that a severe infection during pregnancy increases the risk of a child developing autism spectrum disorder (ASD), which has been supported in mouse models. Notably, treatment with a serum metabolite could cure the disorder and reduce ASD-like symptoms in mice.

Pedersen also briefly discussed the effects of antibiotic use on gut microbiota, warning that the use of antibiotics leads to a microbial profile with increased capacity to extract energy from food. Further, antibiotic use in early childhood increased the risk of childhood obesity (Cho et al., 2012; Liou and Turnbaugh, 2012).

New DNA methods may hold the key to understanding more about the microbiome. The MetaHit project ( $\mathrm{Li}$ et al., 2014) aims to establish a reference catalog of bacterial genes, mapping these to individual bacterial genomes and to cluster these into Meta Genome Units. To date, this project has identified in excess of 10 million genes, only 10\% of which may be mapped to known genomes. A MetaHit study of 292 individuals found that individuals low in 'richness' had increased lipids, weight, and number of proinflammatory bacterial species. Further, roughly one-fourth (40\%) of the patients showed a severe reduction of gut microbiota and experienced a significant increase in disorders (Le Chatelier et al., 2013).

Pedersen discussed briefly the idea of gut bacteriotherapy, arguing that, along with probiotics and a healthy diet, there may be further bioactive bacterial compounds with therapeutic or disease-preventing effects. To exploit this fully, we need proof that the altered bacteria truly cause the disorders as well as novel preclinical models to test the efficacies of bacteriotherapy.

A question was posed on the potential effect of saccharine use on gut flora and on insulin resistance (Suez et al., 2014). Pedersen noted that this study does observe a major change in microbiota because of saccharine use and that this effect is validated in a rodent model. Four of seven human volunteers also became very glucose intolerant and showed a large change in flora. When stool from these patients was transplanted into mice, the effect was reproduced. This implies that we can assume that saccharine use at this level may induce severe glucose intolerance. However, levels of saccharine use studied in this paper are the maximum FDA-approved levels and likely to far exceed normal daily use.

\section{Dr Preben Bo Mortensen (University of Aarhus, Denmark) - epidemiology in psychiatric genetics: putting the $E$ into $G \times E$ (reported by Zuzanna Misiewicz)}

Dr Preben Bo Mortensen (University of Aarhus, Denmark) presented his talk on the Danish total national birth cohort study called 'The Initiative for Integrative Psychiatric Research' Project - iPSYCH. 
The iPSYCH project studied five specific mental disorders: SCZ, bipolar disorder (BD), autism, attention-deficit hyperactivity disorder (ADHD), and depression. The main goal of the project is to identify the causes, both genetic and environmental, underlying the abovementioned disorders, to offer more personalized treatment to patients.

Dr Mortensen began his presentation by reminiscing about Danish psychiatrist Erik Strömgren. In the 1920s, Strömgren conducted a survey of mental illnesses on Bornholm, which is the home island of Mortensen. As one of the first psychiatrists, Strömgren realized the ideal conditions for research on epidemiology of genetic disorders in a geographically isolated population, but more importantly, a population built on open-mindedness and trust (Strömgren, 1982). Almost 40 years later, an initiative to create an electronic database, the nationwide Psychiatric Central Register, was brought to life by Annalise Dupont. By creating the database, she laid a foundation for the intertwined relationship between genetic and epidemiology studies in Denmark and, therefore, also the iPSYCH project (Mors et al., 2011).

The iPSYCH project uses data on Danes born after 1955, with a specific subcohort of people born since 1981, from which neonatal dried blood spots are available, providing information on genetic, epigenetic, and other biomarkers. The retrieval of the samples as well as DNA amplification of the initial 80-85000 cases and controls has already been completed and currently large-scale exome sequencing is taking place.

In his presentation, Dr Mortensen highlighted the specific ideas of the iPSYCH project, such as the integration of epidemiological, genetic, and experimental studies not only within a single disease but also between them. Furthermore, he spoke on the strong connections between numerous nationwide registers existing in Denmark, allowing the researcher to use the full potential of the data by being able to follow the patients and controls over time (Schmidt et al., 2014). Despite the impressive advantages, the iPSYCH project also presents some drawbacks: only clinical diagnoses of patients are available and no further phenotypic data can be collected as there is no possibility of recontacting the patient. As Mette Ebbesen (Aarhus University), a member of the Ethical Advisory Group for iPSYCH project, pointed out in the discussion following the presentation, autonomy of the patients is respected and the initial consent provided by the parents at the child's birth can later be withdrawn.

Subsequently, Mortensen presented examples of how the collected data can be used. One such example was a study carried out by Borglum et al. (2014), in which a GWAS of maternal cytomegalovirus infection suggested a new SCZ locus (Borglum et al., 2014). The study analyzed all individuals born in Denmark after 1981 and diagnosed with the disease. Furthermore, Mortensen introduced a new study (VIA 7) of 600 7-year-old children, either of parents with $\mathrm{SCZ}$ or $\mathrm{BD}$, and controls. Elaborate neuropsychological information is available on all of those 600 children. This study will enable us to identify specific endophenotypes or indicators of vulnerability for the above-mentioned diseases and, therefore, also to develop methods of early intervention and prevention. 
In conclusion, as shown by Dr Mortensen, the many dimensions of iPSYCH offer great opportunities for research as environmental factors and epidemiological information significantly contribute toward the risk of mental disorders.

The use of genetics to study functions and dysfunctions of the brain - speaker: Dr Kári Stefánsson (reported by Martin Tesli)

Dr Kári Stefánsson (deCODE genetics) discussed how molecular genetics can be used to increase our knowledge of the workings of the brain. He presented some examples from his own research at deCODE genetics, where more than 135000 individuals have been chip typed. Two main approaches have been particularly successful. The first is to submit a large number of Icelanders to genetic and cognitive testing to identify variants associated with various aspects of cognitive function. The other is to investigate the ways in which genetic risk variants for neuropsychiatric disorders influence cognition in healthy individuals. As examples of the latter method, he mentioned studies of SCZ, autism, and Alzheimer's disease (AD). In one recent report, it was shown that CNVs conferring risk to SCZ and autism were associated with cognitive deficits in healthy individuals. In particular, control participants carrying a chromosome 15q11.2 deletion had a history of dyslexia and dyscalculia, in addition to alterations in brain structures similar to those observed in firstepisode psychosis and dyslexia (Stefánsson et al., 2014). Stefánsson suggested that these findings indicate that cognitive abnormalities might be an underlying mechanism in SCZ as well as a consequence of the disorder. He also presented recent findings linking genetic risk for $\mathrm{SCZ}$ and BD to creativity. For this purpose, his research group made use of the Psychiatric Genomics Consortium's (PGC's) available data on common sequence variants that predispose to these disorders. They found that polygenic risk scores (PRSs) on the basis of these data were significantly higher in unaffected writers, musicians, painters, dancers, and accomplished chess players than in matched control participants, but this was not true for 20 diseases chosen as negative controls. These findings are in line with previous reports from the Stefánsson group - for example, that a common variant in the nicotinic acetylcholine receptor gene cluster on chromosome 15q24 has an effect on smoking quantity, nicotine dependence, and the risk of smoking-related diseases (Thorgeirsson et al., 2008).

\section{Dr Michael Meaney (McGill University, Canada) - parental effects on the epigenome (reported by Kate Wolfe)}

Dr Michael Meaney (McGill University, Canada) explores the mechanisms by which childhood experiences, particularly maternal care, can be biologically embedded in the form of epigenetic modification and have enduring effects across the lifespan.

Meaney began with an overview of his work with rodents, where he investigated natural individual differences in maternal licking/grooming (LG) behaviors and the hypothalamicpituitary-adrenal (HPA) axis response to stress. He found that offspring of high LG mothers showed increased expression of glucocorticoid receptors (GRs) in the hippocampus, resulting in tighter regulation of the HPA axis in response to stress. These differences can be reversed by cross-fostering of rat offspring, confirming that maternal care is driving the differential expression patterns beyond genomic-based inheritance (Weaver et al., 2004). 
Further work was carried out to understand the biological underpinnings of the differential GR expression. Analysis of the GR gene led to identification of an exon $1_{7}$ promoter sequence that was expressed at higher levels in the hippocampus of off-spring of high LG mothers. Sequencing of the exon $1_{7}$ promoter indicated a perfect consensus sequence for transcription factor NGFI-A. Increased occupancy of NGFI-A at the exon $1_{7}$ promoter was also observed in adult offspring of high LG mothers, again serving to increase GR expression and regulate the HPA response to stress (Meaney and Szyf, 2005).

The transgenerational impact of maternal care and influences on the glutaminergic signaling pathway were also explored. The ERa, which is expressed in the medial preoptic area, showed increased expression in high LG offspring and the offspring themselves became high LG mothers. The ERa $1 b$ promoter region was implicated as a key site for differential methylation, in particular, the region that binds to Stat5b transcription factor, which is known to regulate ERa expression (Champagne et al., 2006). Reduced methylation of the GRM1 gene, which encodes the type I metabotropic glutamate receptor (mGluR1), was found in the hippocampus of adult rodents raised by high LG mothers. Increased MGluR1 mRNA and protein expression in these offspring serves to regulate the postsynaptic impact of glutamate (Bagot et al., 2012).

Meaney examined comparable processes in humans. Adult hippocampal postmortem tissue was acquired from suicide victims and controls and methylation at the ortholog of the rodent exon $1_{7}$ promoter sequence, the exon $1_{\mathrm{f}}$ sequence, was analyzed. An increase in GR transcripts, including exon $1_{\mathrm{f}}$, was found in the brain tissue of controls compared with suicide victims. When this was correlated with various measures of psychopathology, it was found that GR and exon $1_{\mathrm{f}}$ expression was only decreased in the brain tissue of suicide victims with a history of child abuse. As in the rodent model, this was associated with increased methylation of the exon $1_{\mathrm{f}}$ promoter, decreased NGFI-A transcription factor binding, and lower regulation of the HPA axis. This is supported by previous research, which has shown that children who have been maltreated show an increased HPA response to stress (McGowan et al., 2009).

Epigenetic changes were also examined as a function of early development across the whole genome. A birth cohort from Singapore was investigated for methylation status utilizing the Illumina 450k differential methylation array and information was gathered on various measures of maternal care. Brain-derived neurotrophic factor (BDNF) was explored as a candidate gene. For offspring with the rs6265met/met BDNF genotype, $10 \%$ of all variably methylated CpGs were associated with prepartum maternal anxiety scores. Subsequent brain imaging showed that CpGs associated with maternal anxiety in the met/met group were mostly associated with right amygdala volume, whereas in the hippocampus, the association was stronger for the other genotypes. This would suggest that there is genome-wide environment interaction on the methylome, which expresses itself in a regionally specific manner in the brain.

Dr Meaney concluded that longitudinal studies will be key to deciphering the complex interface DNA methylation forms for imprinting dynamic social factors, such as maternal care, on a reasonably fixed genome. 
Plenary panel session - pathways to therapy and prevention (reported by Monica Aas)

Raimund Buller (Lundbeck) described the industry perspective on pathway to therapy and prevention in psychiatry. Current antipsychotics mainly work on positive symptoms, targeting dopamine, not targeting cognitive and negative symptoms, which may be more related to the long-term outcome of these patients.

Steve Hyman (Broad Institute) emphasized that we lacked new molecular targets and mentioned the challenges in using the DSM gold standard for this purpose.

\section{Dr Daniel Geschwind (University of California, Los Angeles) - integrative genomics and the neurobiology of autism spectrum disorders (reported by Monique van der Voet)}

Professor Daniel Geschwind (University of California, Los Angeles) spoke about ASD and how to navigate the complex road from genes to function in a heterogeneous disorder. No major gene accounts for over $1 \%$ of autism and over 1000 genes are estimated to contribute toward ASD. Three major themes below were addressed in the talk:

\section{Despite the etiological heterogeneity, is there a shared pathology in the} autism spectrum disorder brain? If there is, is it specific?-The study of differential expression in ASD cortical regions showed a shared molecular pathology (Voineagu et al., 2011). Clustering based on the top-200 genes showed decreased expression of synaptic signaling genes and increased inflammatory pathway expression. The network structure was robust and reproducible; this provides strong evidence for convergent molecular abnormalities in ASD. For the last 3 years, the lab has worked to replicate the finding in a larger set of samples (113 autism/control brains) using RNAseq. A robust gene expression pattern is shared by $60 \%$ of autism cases, including a recurrent, rare genetic form of ASD (15q11--q13 duplication). This for the first time defines one aspect of a shared molecular pathology of autism. His lab is now working on a cross-disorder analysis to test how the pattern is preserved in other datasets such as SCZ.

\section{Pathology cannot be confused with causality. Do autism risk genes converge} on specific pathways and how do they map on normal brain development?The ASD genes were mapped to cortical development using BrainSpan RNAseq data. Two coexpression modules were found that are highly enriched for rare de-novo variants (Parikshak et al., 2013). Fragile X mental retardation protein (FMRP) targets are specifically enriched in the modules, which confirms that FMRP may be connecting a variety of developmental processes (Iossifov et al., 2012). Microarray data from macaque show that the genes are specifically enriched in neurons of the superficial lamina. More specific data sets point to upper layer glutamatergic neurons. This is consistent with a cognitive science model that part of autism is a long-range disconnection of higher-order cortical association areas, which would be served by these superficial layers.

\section{How can we begin to model autism in Vitro to develop therapeutics?-}

Geschwind explained the importance of understanding functional processes that are modeled with fidelity in Vitro before beginning to knock genes out. Cultured primary human neural progenitor cells (phNPCs) from acute fetal brain were discussed. After 12 weeks of 
differentiation, these phNPCs match cortical developmental periods up to late mid-fetal periods, with strongest correspondence to the transition between the ventricular zone or the sub-ventricular zone to subplate and inner cortical plate (Stein et al., 2014). Many functional protein modules are conserved and there is a strong recapitulation of in-vivo development. However, it has to be recognized that differentiated phNPCs constitute fairly immature synapses. Similar analyses of iPSCs showed considerable variability in neuronal differentiation and the iPSC-derived neurons were also very immature. To model late-onset brain disorders, the maturation process of these in-vitro neurons needs to be encouraged.

In summary, despite the genetic etiologic heterogeneity in autism, there seems to be some convergence. Network context can enable in-vitro and in-vivo modeling and ultimately drug discovery.

\section{Symposia sessions}

\section{New data on the genetics of attention-deficit hyperactivity disorder (reported by Joanna Martin)}

Dr Anders Børglum (Aarhus University, Denmark) presented a status report of ongoing GWAS analyses of Danish population ADHD cases and controls on behalf of the Integrative Psychiatric Research (iPSYCH) research group. This GWAS utilizes blood spot data from the Danish Newborn Screening Biobank, which is a unique resource for biological analyses. Using personal identification numbers, information from the Danish Psychiatric Central Research Register was used to identify $n=18726$ individuals diagnosed with ADHD and link them with their genetic data. A population control group of over 30000 individuals screened for psychiatric problems was also selected. The samples are being genotyped on the PsychChip by the Broad Institute (USA), and the first data freeze consists of $n=4186$ ADHD cases and $n=5652$ controls after standard PGC quality control procedures. The current GWAS included $~ 8.35$ million SNPs after quality control (QC). No SNPs reached genome-wide significance, but some of the top hits included an SNP within the gene FGFR1 on chromosome 8 and another SNP near the gene PGM2 (chromosome 4). Efforts are underway to genotype the remaining Danish ADHD samples and to integrate these data with the PGC ADHD sample. In addition, data for $n=3755$ ASD samples are also available from the Danish Newborn Screening Biobank and a joint ADHD and ASD GWAS also showed no significant SNPs. These Danish samples are likely to represent a considerable contribution to future PGC analyses.

Dr Benjamin Neale (Massachusetts General Hospital, USA) reported the latest results of the GWAS meta-analysis of ADHD, which builds on previous samples (Neale et al., 2010) and now includes additional cases from the Danish iPSYCH study (see above) as well as selfidentified ADHD cases and controls from the 23andMe personal genomics company. The current total sample included in the latest meta-analysis consists of $n=14840$ cases and $n=$ 76029 controls. Although one SNP passed the threshold for genetic association, the imputation score for this SNP was very low and it appeared to be an isolated signal in that locus; thus, it is likely to be a spurious result. However, the QQ plot showed inflation as would be expected under a polygenic architecture of ADHD. Neale then outlined a recent method that he developed with Dr Brendan Bulik-Sullivan (UNC School of Medicine, USA) 
and colleagues that uses linkage disequilibrium (LD) score regression to distinguish the effects of population stratification from true polygenicity in GWAS of complex diseases such as psychiatric disorders (Bulik-Sullivan et al., 2015). This method estimates the heritability of disease from common SNPs and is computationally more efficient than the commonly used genome wide restricted maximum likelihood GREML approach (Yang et al., 2011) as it uses only the summary statistics of GWAS. The estimation of SNP heritability for ADHD using this method ranged from approximately 21 to $33 \%$ depending on the subset of the sample analyzed.

Dr Beth Wilmot (Oregon Health \& Science University, USA) outlined some of the major issues of pathway analytic approaches to parsing the biology of a complex disorder such as ADHD, including the use of gene ontologies (GOs) to define pathways, and the complexities of mapping SNPs to genes. There are now many different pathway analytic algorithms and methods, which have different features, biases, and strengths. Wilmot presented data using six different methods that utilize individual genotype data in two samples: a German ADHD case-control sample (Hinney et al., 2011) and the first PGC ADHD meta-analysis sample (Neale et al., 2010). No biological pathways were found to be significant across all six methods and most pathways found to be nominally significant were only detected by one or two of the methods. However, the top pathways picked up by 3-5 of the methods in both of the samples included those related to potassium channels, FGFR2b ligand binding and activation, voltage-gated potassium channels, and synaptic vesicle trafficking. Wilmot stressed the importance of manually curated pathways and outlined plans to use imputed data and covariates, and include CNV data in future analyses.

Dr Jan Haavik (University of Bergen, Norway) presented data from several of his group's recent studies using different methods to investigate the disease mechanisms of ADHD by examining candidate genes, which could lead to future treatments. $\mathrm{CDH} 13$ is a gene that codes for the brain-expressed protein T-cadherin and was first implicated in ADHD through linkage analyses (Zhou et al., 2008). Haavik and colleagues sequenced this gene in adults with ADHD (from the IMPACT sample), but found no strong effects, although they did find a decrease in serum levels in individuals with missense variants in this gene. One possible mechanism through which missense variants may affect protein function is by affecting the folding; indeed, studies of the three-dimensional structure of mutations in the $\mathrm{TH}$ protein confirm altered substrate specificity (Fossbakk et al., 2014). Using another approach, Haavik and colleagues suggest that mis-folded proteins (specifically, TPH1 and TPH2, other candidate ADHD genes) may be rescued through the use of pharmacological 'chaperones' (Calvo et al., 2010). Haavik stressed that multiple approaches using the latest statistical tools and methodology (e.g. biochemical studies, in-silico modeling, and molecular systems biology approaches) will be needed to understand the etiology of ADHD.

Dr Barbara Franke (Radboud University Medical Center, the Netherlands) concluded the session by also highlighting the importance of using multiple approaches in the study of ADHD and the collaborative contribution of population-based samples such as the $\mathrm{PSSYCH}$ Danish sample to the PGC ADHD sample. Franke pointed out that although we still do not have any genome-wide significant 'hits' for ADHD, the growing sample sizes present a useful opportunity for downstream analyses - for example, by examining the effect of 
candidate pathways in relation to symptoms (Bralten et al., 2013). Franke also highlighted the importance of using multiple methods to study this complex condition - for example, by looking at delays in brain maturation using neuroimaging or by using knockout animal (e.g. zebrafish) models to study the effects of candidate risk genes.

\section{Telomeres and telomerase in mood disorder: markers for risk and treatment? (reported by Claudia Pisanu)}

Professor Catharina Lavebratt (Karolinska Institutet): telomeres prevent loss of genomic content during replication of linear DNA. Telomere length (TL) decreased with cellular age and the shortening rate is about $14-60$ base pairs/year. However, this shortening is, to some extent, reversible through the telomerase enzyme, which is expressed in germ cells, stem cells, and undifferentiated leukocytes (thus in areas of neurogenesis). Telomeres are sensitive to reactive oxidative molecules produced by oxidative stress in the cell. In postmitotic cells, in which telomerase is not expressed, increased oxidative stress induced by inflammation or increased HPA-axis activity causes excessive telomere shortening. Excessively short telomeres induce necrosis/apoptosis and, as a result, cell loss and tissue dysfunction. Short leukocyte telomere length (LTL) has been related to higher mortality (Cawthon et al., 2003), age-related diseases, and psychological stress-related diseases, such as cerebrovascular diseases, cardiovascular diseases, type 2 diabetes, dementia, and depression (Shalev et al., 2014; Verhoeven et al., 2014a). Genetic variation (Codd et al., 2013), DNA methylation in subtelomeric regions (Buxton et al., 2014), sex (Verhoeven et al., 2014a), and life style have been reported to influence variation in LTL. Inflammation, dysregulation of HPA axis, and autonomic stress have all been associated with shorter LTL in individuals with depression and anxiety (Révész et al., 2014). Professor Lavebratt also provided an overview of studies that associated LTL with psychiatric disorders. In particular, MDD (Verhoeven et al., 2014a) and a high number of depressive episodes in MDD and BD (Wolkowitz et al., 2011; Martinsson et al., 2013) have been associated with shorter LTL. Finally, some studies have proposed a telomere-protecting effect of psychotropic drugs (Wolkowitz et al., 2011; Martinsson et al., 2013).

Professor Brenda Penninx (VU University Medical Center) presented data from the Netherlands Study of Depression and Anxiety (NESDA), one of the largest studies to measure LTL in psychiatric patients (Verhoeven et al., 2014a). MDD is strongly associated with the onset of many different aging-related somatic conditions (Penninx et al., 2013). In this sense, LTL is a most interesting biomarker of cellular aging to investigate in MDD patients. The NESDA is a large naturalistic cohort study that recruited over 2900 participants, including MDD patients, patients with anxiety, and healthy controls (Penninx et al., 2008). Determination of LTL with quantitative CR showed a negative correlation with age $(P<0.001)$ and a longer LTL in women $(P<0.001)$. Compared with healthy controls, sociodemographic-adjusted LTL was shorter among MDD patients in remission $(P=0.014)$ and current MDD patients $(P=0.012)$. Within MDD, severity and duration were associated with shorter LTL (Verhoeven et al., 2014a). However, late-life depression was not associated with shorter LTL (Schaakxs et al., 2015). Compared with healthy controls, LTL was shorter among patients with current anxiety, but not among patients with remitted anxiety (Verhoeven et al., 2015). Possible pathways linking MDD and telomere shortening include 
chronic hyperactivity of the HPA axis, autonomic stress, and low-grade inflammation, especially when all these dysfunctions occur together (Révész et al., 2014). An important question is whether cellular aging can be reversed through a therapeutic intervention (Verhoeven et al., 2014b). Psychotropic medications did not seem to impact LTL in the NESDA cohort.

Yabin Wei (Karolinska Hospital) showed that long-term lithium treatment in BD has been associated with longer TL (Martinsson et al., 2013). Telomeres are maintained by the enzyme telomerase, which, in adult brain, is still expressed in the hippocampus. In murine studies, inhibition of hippocampal telomerase TERT subunit expression induced neuronal excitotoxicity, apoptosis, and depressive behavior (Zhou et al., 2011). The SNP rs2736100 of the human TERT gene has been associated with TL in cohorts of healthy adults (Codd et al., 2013). Yabin Wei discussed the results from a recent study showing shorter telomeres, decreased TERT expression, and telomerase activity in the hippocampus of the Flinders Sensitive Line rats, a genetic rat model of depressive-like behavior (Wei et al., unpublished results) compared with the Flinders Resistant Line (Overstreet and Griebel, 2005). A 6-week lithium treatment increased TERT and telomerase activity, but not TL. The second part of this study was carried out in humans and showed that TL in DNA extracted from saliva is shortened in adults with depression without exposure to childhood adversity compared with controls. Moreover, rs2736100 was significantly associated with unipolar depression and with the number of depressive episodes in BD patients.

Professor Adolfo Sequeira (University of California Irvine) reported results supporting the involvement of stress response in psychiatric disorders. In particular, functioning of the HPA axis, expression of the enzyme spermidine/spermine $N(1)$-acetyltransferase 1 (SAT1), the rate-limiting enzyme in the catabolism of polyamines (Sequeira et al., 2006), and expression of metallothioneins (Sequeira et al., 2012) are all altered in psychiatric disorders. Moreover, shorter LTL has been associated with many diseases, suggesting reduced neuroprotection against the physiological effects of stress in psychiatric disorders. To elucidate possible regional differences in the brain of patients with psychiatric disorders and controls, TL was measured in five different brain regions in a sample of 40 individuals (Sequeira et al., unpublished results). The study confirmed that TL is not strongly correlated with age in the brain, but showed significant differences in TL across brain regions. In particular, MDD patients showed a significant decrease in TL, specifically in the hippocampus. Direct RNA analysis on the same patients using the NanoString platform suggested a possible role of GR and corticotropin-releasing hormone gene expression in stress responses through regulation of telomere maintenance gene expression.

\section{Beyond Bonferroni: large-scale inference for complex disorders (reported by Laura Huckins)}

Peter Visscher (University of Queensland) began the session by suggesting that we are now in a time beyond standard GWAS; we have more loci and far better estimates of effect sizes. Visscher suggests that it is time to adopt a new null hypothesis: that all variants affect all traits. The question then becomes not 'Is there variance?', but instead 'Do we see significantly more variance than might be expected by chance?' 
Anders Dale (UCSD) proposes a new model that works with Visscher's new null hypothesis, allowing for small ubiquitous effect sizes, implying that every SNP has at least a low association with the trait. This model is a straightforward bivariate and multivariate extension of Gaussian mixture modeling. When considering real data, we see that a standard model is unable to fit small effect sizes well. Conversely, a polygenic model effectively sets large effect sizes to zero. Dale's proposed model accounts well for both types of effect sizes and is thus able to better model the true genetic architecture. Further, the model is able to model pleitropy, increase power for discovery, and may help to identify biological mechanisms.

Ole Andreassen (University of Oslo) spoke on 'Leveraging Pleiotropy to Improve Gene Discovery and Effect Size Estimation'. Pleiotropy may be leveraged to aid gene discovery, boost study power, and increase the replication rate. Andreassen outlines a method using conjunctional false discovery rate (FDR), rather than conditional FDR, essentially using variants conditioned on one trait to discover variants in a second trait. For example, replication rates in SCZ may be increased by conditioning on blood lipids (Andreassen et al., 2013), triglycerides (Andreassen et al., 2013), multiple sclerosis (Andreassen et al., 2015), and college attainment (Lencz et al., 2014). However, this is not a global effect; for example, conditioning on ulcerative colitis does not improve $\mathrm{SCZ}$ replication rates, implying that there must be some biological link between disorders to boost discovery or replication.

Sang Hong Lee (University of Queensland) discussed combined analysis of psychiatric disorders using two new programs, STGBLUP and MTGBLUP (single/multitrait genomic best linear unbiased prediction), which use genotypes to construct a linear mixed model and thus estimate SNP effects. PGC cross-disorder data were used as a discovery set, whereas independent SCZ, BD, and MDD samples were used as a validation. After excluding any related individuals, this validation set included $\sim 60 \mathrm{k}$ individuals. Genetic heterogeneity between data sets was tested using a principal components analysis (PCA) and a genetic variance analysis.

Prediction accuracy in the validation set was better when using a multitrait approach. Risk prediction scores were low (ranging from 0.2 for $\mathrm{SCZ}$ to $<0.1$ for MDD), although stratifying on SNPs involved in central nervous system (CNS) improved SCZ and BD values slightly. This filtering approach improves risk prediction accuracy equivalent to adding 5000 samples to the SCZ and BD studies or 10000 to the MDD study.

Andrew Schork (UCSD) discussed an attempt to identify functional categories that are more likely to contain polygenic effects. All SNPs in the 1000 genomes project were annotated as $5^{\prime}$ UTR, exon, intron, 3'UTR, and intergenic (Schork et al., 2013). As LD-genotyped SNPs may act as proxies for potentially causal variants, LD-informed scoring was used to update these categories if necessary. This method was tested across 14 GWAS, all of which were subjected to stratification because of polygenic effects. The $5^{\prime}$ UTR annotation was significantly inflated among all of these studies, whereas the intergenic region was always underinflated. The functional annotation also affected the likelihood of replication; at a $10^{-3}$ cut-off, only $25 \%$ of intergenic variants replicated. However, replication increased to $75 \%$ 
for $5^{\prime}$ UTR SNPs using the same cut-off. This implies that differences in enrichment according to functional annotation may be a true finding.

\section{Shared genetics between depression and comorbid physical conditions (reported by Lynsey Hall)}

Following a strong lead: functional investigation of genome-wide association study signals for schizophrenia (reported by Jose Estrada)-As the number of candidate genes increases with each published GWAS, researchers are now using a number of techniques to assign function to individual candidates.

Dr Nick Bray (Kings College London, UK) presented work that looks at identifying the direct effects of risk alleles on gene output utilizing an allele-specific discrimination assay. Specifically, deviations from a 1:1 expression ratio of the risk versus nonrisk allele in heterozygous individuals detect those variants that affect gene output. To control for indirect effects, homozygotes for each allele are analyzed and must have a $1: 1$ expression ratio. ZNF804A analysis shows deviation from risk versus nonrisk allele expression in secondtrimester fetal brain tissue, but not first-trimester or adult brain. NT5C2 and CACNA1C analysis identified gene expression distortions within the PFC and the cerebellum, respectively.

Dr Matt Hill's (Cardiff University, UK) research interest lies in understanding the downstream functional consequence of risk variants that affect gene expression. Using two neural progenitor cell (NPC) lines in combination with small interfering RNA to knock down target gene expression, he can then assay for downstream effects using microarrays and GO analysis. Hill's initial investigations focused on three genes that are downregulated in SCZ and regulate gene output: ZNF804A, a member of the zinc finger protein family capable of binding RNA and DNA; miR-137, a microRNA that potentially targets and represses many genes; and the transcription factor 4 (TCF4). Knockdown of ZNF804A in NPCs suggests that it regulates 154 candidate genes. GO analysis shows many of these genes to be involved with cell adhesion. miR-137 knockdown analysis shows a smaller number of candidates, but focuses predominantly on genes involved in neurodifferentiation. TCF4 knockdown analysis indicates a number of candidates involved in cell cycle regulation.

Dr Emmanuel Schwarz (Central Institute of Mental Health Mannheim, Germany) is investigating neural mechanisms linked to GWAS candidates in SCZ using functional MRI and several cognitive endophenotypes associated with SCZ participants and their first-degree relatives. Schwarz reports that individuals with the SCZ GWAS-associated risk allele for neuregulin 1 also show dysfunction in the ventral striatum when completing a reward/ salience task. Individuals with the GWAS-associated risk allele for ZNF804A completing the two back working memory task show abnormal dorsal lateral PFC-hippocampal connectivity. Individuals with a risk allele for SCGN within the GWAS-associated major histocompatibility complex (MHC) locus show defects related to hippocampal volume/ connectivity. Individuals with a risk allele for CACNA1C show defects in hippocampus and subgenual anterior cingulate activity when completing a face recognition/episodic memory task. 
Dr Andrew McIntosh (University of Edinburgh, UK) is investigating the overlap between polygenic psychiatric diseases (SCZ, alcohol abuse, and autism) and cognitive ability which itself is a polygenic phenotype. Negative correlations with the three disorders and the IQ score have been observed; this is considered to be a genetic association based on twin and bivariate studies. PRSs for SCZ, alcohol abuse, and autism were generated in population-based samples with corresponding GWAS and cognitive data. PCA was utilized to identify correlations between the various cognitive tests and the polygenic score for each disorder. PRSs for SCZ and alcohol abuse correlated negatively with cognitive ability. However, in the case of autism, PRSs were associated positively with cognitive ability.

The investigators of this panel are at the forefront of assigning molecular and cellular, spatial, temporal, and cognitive function to GWAS-identified candidate genes.

\section{New Findings from the Enhancing Neurolmaging Genetics through Meta- Analysis (ENIGMA) Consortium (reported by Gabriëlla Blokland)—Dr Derrek} Hibar (Imaging Genetics Center, University of Southern California, USA) introduced the Enhancing NeuroImaging Genetics through Meta-Analysis (ENIGMA) consortium, which aims to learn more about psychiatric disorders by studying changes in the brain and linking those to genetic variation. ENIGMA is a collaborative network of researchers working together on a range of large-scale studies that integrate data from many institutions worldwide. The ENIGMA consortium currently encompasses 185 institutions, with over 300 investigators from 33 countries. ENIGMA is organized into Working Groups that tackle questions in neuroscience, genetics, and medicine. Active working groups include those on ADHD, autism, addiction, HIV, MDD, obsessive-compulsive disorder, BD, SCZ, and diffusion tensor imaging (DTI). Currently, these groups are working on finding the best endophenotypes - that is, which brain regions are the best biomarkers for distinguishing patients from controls, but ultimately the goal is to run GWAS. Results from the ENIGMA1 pilot project, a GWAS meta-analysis of hippocampal volume and intracranial volume (ICV) in a combined discovery and replication data set of $n \approx 21000$, were published in 2012 (Stein et al., 2012). Crucial to the success of this study - and of ENIGMA - is that MRI processing and genetic analysis were carried out at each site using a set protocol, after which each site submitted genetic association statistics for meta-analysis. Identical data-processing across sites and strict QC make it less likely that the lack of effect is because of noise introduced by merging heterogeneous data sets. One genetic variant, located between HRK and FBXW8, explained a significant proportion of the variance in hippocampal volume; it was associated with a $1.2 \%$ decrease in the average hippocampus volume per risk allele. In addition, three significant loci for ICV were identified. ENIGMA joined forces with the CHARGE consortium (Bis et al., 2012) to replicate each other's hits and found that the top five variants from each structure were exactly the same. Interestingly, this study did not confirm many of the classical candidate genes for brain structure, such as BDNF and APOE.

Dr Sarah Medland (QIMR Berghofer Medical Research Institute, Australia) discussed ENIGMA's current main project, a GWAS of seven subcortical brain structures (ENIGMA2), in a discovery sample of $n=13688$ (30 cohorts) and a replication sample of $n$ $=15748$ (18 cohorts). Across phenotypes, five genome-wide significant loci $(P<7.1 \times$ $\left.10^{-9}\right)$ were identified and the replication added another five loci. Significant loci were 
associated with $0.5-1.5 \%$ differences in volume per risk allele, explaining $0.2-0.52 \%$ of the phenotypic variance. Effect sizes are no greater for imaging endophenotypes than for any other complex physical trait. This challenges the original assumption that endophenotype effect sizes would be greater than disease phenotype effect sizes because they are closer to the biology. According to GCTA, full GWAS results explained 7-15\% of the phenotypic variance in the subcortical volumes after correction for covariates. Importantly, the ENIGMA1 hits for hippocampus and ICV were supported, and the associations did not disappear when excluding all patients from the analyses. Most hits were structure specific; there was not a lot of genetic overlap because of the orthogonalizing effect of correction for ICV. Multivariate cross-phenotype analyses using the TATES method (van der Sluis et al., 2013) confirmed the univariate results, but no additional loci reached genome-wide significance. At a multiple-phenotype-corrected genome-wide significance threshold, no significant variants were identified for the thalamus, pallidum, amygdala, caudate nucleus, or NAc, although several strong signals for the amygdala and NAc will likely reach significance with increased power. However, four genome-wide significant variants were identified for the putamen (which were also replicated in the CHARGE consortium data). One of the loci identified is $14 \mathrm{q} 22$, a region with many epigenetic markers, which houses the KTN1 gene. KTN1 codes for kinectin, a protein found in the dendrites (but not axons) of neurons. Gene expression differences for KTN1 in frontal and putamen brain tissue confirm localization of the GWAS signal in KTN1 and developmental expression data indicate that these effects likely begin in the late prenatal stage and continue through adulthood. Finally, support for the validity of this finding comes from an animal study, in which increased KTN1 expression in the striatum of 31 isogenic BXD strains of mice was associated with larger striatal volumes.

Dr Ole Andreassen (University of Oslo, Norway) leads the ENIGMA Bipolar Disorder working group. He pointed out that many of the brain effects in psychiatric disorders are very subtle; thus, we need large samples to identify them. Although ENIGMA was initially formed with the goal of undertaking imaging genetics, it has evolved into a consortium that also analyzes patient scans using the same processing pipeline so as to be able to identify the best endophenotypes for various neuropsychiatric disorders. In a BD case-control metaanalysis of the seven subcortical brain volumes, accounting for age, sex, and ICV, the largest effects of $\mathrm{BD}$ were detected in the hippocampus, thalamus, and amygdala (volume reductions), and lateral ventricles (increase). No striking differences were found between BD subtypes I and II, although hippocampus and amygdala volumes were significantly different. There was a highly significant effect of lithium use on the thalamus and to a lesser extent on the hippocampus. This is likely not an effect of treatment responsiveness because most patients included in these studies respond quite well to medication. Similar patterns of subcortical abnormalities were observed in SCZ (working group led by Jessica Turner) and MDD(working group led by Lianne Schmaal). In a meta-analysis of more than 2000 SCZ patients and more than 2500 controls, the most striking finding was a reduction in hippocampal volume, followed by significant volume decreases in the amygdala, thalamus, and NAc. There seems to be an effect of chlorpromazine dose equivalent, age of onset, and duration of illness in SCZ (i.e. abnormalities are much less severe in first-episode than in chronic patients). Similarly, in the MDD data ( $n \approx 9000$ cases and $n \approx 7000$ controls), effects 
are partly driven by the reoccurrence rate and age of onset, where higher numbers of major depressive episodes and/or earlier onset are associated with smaller subcortical volumes. Interestingly, $\mathrm{ADHD}(n \approx 1500$ cases and $n \approx 1500$ controls; working group led by Martine Hoogman) is associated with strong reductions in the NAc, amygdala, caudate, and putamen, but not the hippocampus, indicating a different neural mechanism in this disorder.

Dr Neda Jahanshad (Imaging Genetics Center, University of Southern California, USA) discussed multisite genetic analysis of DTI scans by the ENIGMADTI working group. DTI allows us to study the brain's white matter, the bundles of myelinated axons connecting cortical regions. This technique makes use of the fact that the diffusion of water molecules is not uniform across the brain because of anatomical constraints and the myelin that surrounds the axons. Postmortem research has shown reduced myelin content in the brains of psychiatric patients and this is reflected in a DTI-derived measure called fractional anisotropy (FA). However, additional technical challenges are associated with combining DTI data from different sites, such as a greater number of artifacts, and differing numbers of gradient directions, which affect the power to accurately measure diffusion. The ENIGMADTI group attempts to address some of these issues and asks whether it is possible to combine DTI data from multiple sites and obtain consistent measures. Using a streamlined processing and quality control pipeline, Kochunov and colleagues (Jahanshad et al., 2013; Kochunov et al., 2014) processed DTI scans from five family-based cohorts and assessed the heritability of tract-based measures of FA. A few tracts were not reliable or not heritable because of size or registration problems; thus, these were not taken forward to GWAS. The GWAS study focused on the comparison of meta-analysis pooling methods: sample size based versus standard error based. Irrespective of the pooling method, the findings were highly similar. Furthermore, to investigate whether mega-analysis is more powerful than meta-analysis, they compared the two methods for 15 candidate SNPs previously shown to have associations with DTI measures, including a BDNF variant, which was significantly associated with FA in two of the five cohorts. There were no significant differences in the results of the two methods; however, standard errors for each SNP were greater with megaanalysis than with meta-analysis, indicating that meta-analysis can deal better with some of the intersite heterogeneity.

\section{A small window to psychiatric genetics in China (reported by Jie Song)}

Dr Chunyu Liu (University of Illinois at Chicago, USA) opened the session by stressing the global diversity taskforce in psychiatric genetic studies and the aims to promote psychiatric genetic research in developing countries. So far, there are nine psychiatric GWAS in China involving dozens of sites. They call for support from international communities and collaboration, hopefully to turn a small window to an opening door.

Dr Hong Xue (Hong Kong University of Science and Technology, China) reviewed work on the function of GABRB2 in SCZ. Their group first reported the association between SCZ and SNPs in GABRB2 (Lo et al., 2004; Petryshen et al., 2005; Lo et al., 2007a). Later, they reported the functional impacts of SCZ-associated noncoding SNPs in GABRB2 at both mRNA and protein levels (Zhao et al., 2006, 2009). They further showed GABRB2 to be under strong positive selection (Lo et al., 2007b), active recombination (Ng et al., 2010), and 
genomic imprinting (Pun et al., 2011), likely contributed by a human lineage-specific insertion of an AluY transposable element. Moreover, epigenetic regulation of GABRB2 showed its developmental control and disruption in SCZ (Zhao et al., 2012). Most recently, they extended GABRB2 association to social cognitions (Tsang et al., 2013). Their work has improved the current understanding of SCZ and relevant intermediate phenotypes as related to GABRB2.

Dr Yin Yao (National Institutes of Health, USA) presented a study using functional MRI data to investigate the compensatory plasticity mechanism in healthy siblings of treatmentresistant SCZ patients. Compared with SCZ patients, healthy siblings presented connectivity variations involving greater than $60 \%$ of the whole brain regions, whereas they showed modest connectivity alterations in small brain regions in contrast with healthy controls. However, SCZ patients have greater than $40 \%$ comparable brain regions that showed connectivity variations compared with healthy controls and those brain regions partially overlapped with that of a sibling/case study. Moreover, the altered connectivity features numbers between cases and controls that are smaller than those between cases and siblings. Their study indicated that connectivity variations in SCZ patients and their healthy siblings are in different scales or even opposite directions, which may be a form of virtuous compensatory regulation preventing the healthy siblings from becoming ill.

Dr Chao Chen (Central South University, China) presented studies on the regulatory network inferences in psychiatric disorders from different levels. They applied weighted gene coexpression network analysis (WGCNA) to gene expression microarray data, and found that NOTCH2 in network M1A and MT1X in network M3A were associated with SCZ and $\mathrm{BD}$ across two brain regions. Moreover, their study on DNA methylation suggested four novel candidate genes, PIK3R1, BTN3A3, NHLH1, and SLC16A7, for the investigation of neuropsychiatric diseases.

Dr Miaoxin Li (University of Hong Kong, China) introduced gene-based and pathway-based methods (http://statgenpro.psychiatry.hku.hk/limx/kgg/) to efficiently reanalyze GWAS data of psychiatric diseases. Using the data released by the PGC, their knowledge-based secondary analyses in an early meta-analysis of SCZ (Ripke et al., 2013) showed a number of additional interesting genes and pathways that were successfully replicated by a larger meta-analysis of SCZ (Schizophrenia Working Group of the Psychiatric Genomics Consortium, 2014). The same genes showed interesting coexpression patterns in brain-tissue networks. Still, validation in an independent dataset is necessary. This speaker also mentioned that the use of the knowledge-based mining system for GWAS to carry out setbased association analysis for intergenic regions is under development and that epigenomic markers are being used to define functional regions for set-based association analysis. Their study supported the polygenic model of major psychiatric disorders, and it showed that introducing genomic knowledge into conventional statistical genetic analysis is a powerful strategy to characterize risk genes of complex diseases.

Dr Pak Sham (University of Hong Kong, China) closed the session by stating that as a country with the largest population, China should contribute more toward psychiatric genetic studies. With more than 100 risk genes identified for SCZ, studies on what the risk genes 
share in common and how they work together may be one of the main directions in the following years. From a clinical perspective, studying the different endophenotypes may help investigate the complicated etiology of SCZ.

\section{Family genome sequencing in bipolar disorder (reported by Alex Shaw)}

Dr Seth Ament (Institute for Systems Biology, USA) discussed sequencing efforts in BD using densely affected pedigrees. So far, 215 affected individuals, 152 of their unaffected relatives, and more than 1000 population controls have been whole genome sequenced. Although initially he had expected to find highly penetrant 'pseudo-Mendelian' variants within the pedigrees, analyses carried out so far have overwhelmingly pointed to an oligogenic or a polygenic structure. Focusing on genes that are recurrently mutated in BD pedigrees, but not control pedigrees, a number of pathways were found to be enriched including neuronal excitability, GABA receptors, and voltage-gated calcium channels. Examining the contribution of rare variants $[<5 \%$ minor allele frequency (MAF)] overall using elastic net regression, he developed a rare variant burden polygenic score and found that rare variants in 140 genes explained several percent of the risk for BD. Another recurring theme is that rare risk variants are overwhelmingly found in gene-regulatory regions rather than coding regions. Accordingly, he focused on identifying transcriptional networks that rare variants are likely to disrupt collectively. By combining gene expression data from BrainSpan (http://www.brainspan.org) with DNAse I hypersensitivity footprinting data from ENCODE, he constructed a brain transcriptional regulatory network. Using mixed models to test for the association between rare variants within predicted transcription factorbinding sites ( 7000/individual), he found that these explained a substantial fraction of the heritability of BD.

Dr Maja Bucan (University of Pennsylvania, USA) discussed sequencing of a large Old Oder Amish pedigree of 700 individuals. She outlined some of the advantages of studying $\mathrm{BD}$ within the pedigree, including the uniformity of the environment and extensive medical records available. She calculated relatedness using SNP data (from 400 individuals) and performed whole-genome sequencing of trios based around distantly related individuals. She used a combination of methods, including linkage within nuclear families on the basis of microsatellite data, phasing, and imputation, and family-based association testing. Although a number of common, potentially damaging SNPs were identified that were absent in the general population, no genome-wide significant association was identified. Turning to linkage analysis, four peaks were identified with Log of the Odds (LOD) more than 2.8 and family-based association testing was performed within these regions. Although many variants under the peaks are putatively damaging and are novel or rare, there were no genome-wide significant associations at the gene level. These results suggest that linkage peaks mark clusters of small-effect to moderate-effect variation, again underscoring the polygenicity and heterogeneity of BD heritability, even within a relatively genetically homogenous population. The speaker raised the need to enrich phenotypic data by including non-psychiatric comorbidities where known.

Dr William Byerley (University of California, San Francisco, USA) examined a genetic isolate on the island of Palau, Micronesia, that included 203 psychotic cases, 221 unaffected 
relatives, and 125 controls who were genotyped by an SNP chip. The psychotic cases had a range of diagnoses, including SCZ, probable SCZ, and schizoaffective disorder, depressed or bipolar type. On carrying out analyses for regions of homozygosity, there was minimal evidence for highly penetrant recessive or common recessive alleles. Whole-genome sequencing was performed on 26 individuals. Variants were filtered to determine putatively damaging missense variation. After filtering, variants in two genes were identified: PCDH10 and $\mathrm{RAB} 33 \mathrm{~B}$, with variants $\sim 6 \mathrm{Mb}$ apart on the same haplotype. Byerley examined the presence of variants in these genes in other Palauan families and other datasets - SCZ trios and unrelated individuals - and found that they were very rare. It is likely that much larger sample sizes will be necessary to detect the effects of such very rare damaging variations at the individual gene level (Zuk et al., 2014).

Dr Tadafumi Kato (RIKEN, Japan) presented his work in exome sequencing of BD trios. Fifty-nine probands and their parents were sequenced including 28 probands with a firstdegree relative with the disorder and the remainder with an apparently sporadic disorder. This design allowed the stratification of discovered variation into transmitted and nontransmitted categories, and the examination of whether any genetic liability was specific to one of these modes. A GO enrichment analysis was carried out, with "calcium ion binding' identified as a top hit, consistent with pathway analysis within GWAS and previous mechanistic studies showing elevated levels of calcium in blood cells (Kato et al., 2003). The specificity of these categories to BD was confirmed using healthy siblings of patients with ASD and by simulation with synonymous mutations, which are not expected to affect protein function. On comparing the transmitted versus nontransmitted variants, no significant difference was found in the number of damaging or nonsense mutations; however, there was no complete overlap between GO terms enriched in transmitted and nontransmitted variants.

In discussion, Dr Peter Zandi (Johns Hopkins, USA) raised the question of the value of performing family studies, given that 'pseudo Mendelian' variants have largely not been identified in BD as originally anticipated. The speakers of the session raised the point that family studies capture more of a range in phenotypes and comorbidities than case-control studies, which, by design, target only individuals with a definite diagnosis and control individuals without a history of psychiatric illness. Dr Bucan raised the point that families also have a longitudinal value, in that information on individuals has often been collected over a number of years and individuals can be followed up. Dr Ament pointed out that individuals in multiply affected families often have a very high genetic load, an enrichment that is inherently valuable when attempting to identify genetic factors across the spectrum of allele frequencies.

\section{The application of isolated populations in the identification of rare risk variants for psychiatric disorders (reported by Erik Loken)}

Dr Hreinn Stefansson (deCODE genetics) reported findings on CNVs conferring the risk of autism or SCZ and their effect on cognition in Icelandic control samples (Stefánsson et al., 2014). He presented data that fecundity was impaired in carriers of $16 \mathrm{p} 11.2$ deletions. Data in 41643 trios also suggested that more high-impact CNVs have a higher proportion of de- 
novo origins, for example over $80 \%$ of $16 \mathrm{p} 11.2$ deletions were de novo, but less than $20 \%$ were for 16p13.1 duplications. Carriers of the $15 \mathrm{q} 11.2$ deletion were shown to have an increase in the volume of the corpus callosum. Dr Stefansson explained that studying these neuropsychiatric CNVs in normal control patients might be an entry point into the mechanisms of brain function and dysfunction. He drew parallels between relatives of SCZ patients also having cognitive deficits that were partly independent of disease status and the incomplete penetrance of the CNVs. In the future, he plans to use functional MRI to move beyond structure and define the neural systems affected by CNVs.

Dr Aarno Palotie (University of Helsinki) reported on data from studies in the Finnish population. The advantages of the Finnish population for genetic studies are that it had a small number of founders, very low immigration, linguistic cultural isolation, and a rapid population expansion with multiple bottlenecks in the 16th century. Dr Palotie studied SNPs in 36262 Finns and found loss-of-function (Lof) variants in LPA that are more common in the Finnish population and exerted a cardioprotective effect (Lim et al., 2014). He extended this methodology to neuropsychiatric disease and studied 256 Lof variants in 7728 Finnish exomes. His group found that the autism and SCZ risk gene C12orf65 contained Lof variants that were heavily enriched in Kuusamo, an isolated and genetically homogenous part of Northern Finland. They also observed an increase in missense variants in the SCZ risk genes NCAN, KCNB1, and FAM109B.

Dr Todd Lencz (Zucker Hillside Hospital) presented data on a rare variation in an Ashkenazi Jewish case-control SCZ cohort. The Ashkenazi Jewish population went through a population bottleneck 33 generations ago with an effective founder population of 270 . Dr Lencz compared long IBD segments in a cohort of 1000 cases versus 1600 controls, used previously in a GWAS implicating that NDST3 confers a risk of SCZ (Lencz et al., 2013), and found more segments in cases for the MHC locus (10.23 vs. 8.35, $P=0.003)$. For rare variants, the recessive model was the strongest model in this segment with $46 / 858$ homozygous cases versus 24/1616 homozygous controls.

Dr Francesco Lescai (Aarhus University) presented a study of rare and de-novo variations in patients with BD in the Faroe Islands. He studied 28 BD cases and 214 controls with $35 \times$ whole-exome and $6 \times$ whole-genome sequencing. In a meta-analysis with another study by University College London of 2025 bipolar cases and 1358 controls, variants in FBXO21, NOS1, and P2RX7 were significant. The PIK3C2A and BEND4 genes were significant in a gene-based analysis, and NCL and MYL12A were implicated in an analysis using Disease Association Protein-Protein Link Evaluator (DAPPLE) (Rossin et al., 2011). These genes are implicated in neurotransmission and neurodevelopmental hypotheses for BD.

\section{Endophenotypes for gene discovery in major mental disorder (reported by Suhas Ganesha)}

This session was chaired by Dr Andrew McIntosh (University of Edinburgh) and moderated by Laura Almasy (Texas Biomedical Research Institute). Four panelists including the chair presented their findings in the area of using endophenotypes for gene discovery in depression, SCZ, and BD. 
Dr Andrew McIntosh introduced the session with an elaborate description of what endophenotypes mean, methods of measurement, polygenic architecture of these traits, and their importance in gene discovery using linkage and family-based association studies.

He described the use of quantitative endophenotypes as alternatives to clinical bivariate diagnoses in GWAS for MDD. Variables from the General Health Questionnaire (GHQ), Eysenck Personality Questionnaire, Cognitive Battery, measures of Schizotypy, and Bipolar Spectrum were assessed as endophenotypes. The study used two methods of analysis, first involving prioritizing of variables with genetic correlation with MDD and using multivariate GWAS with the vector of these traits. Second, the investigators used PCA of the genetic and phenotypic correlations. Both methods showed convergent results, with Neuroticism, Schizotypal Personality Questionnaire, Mood Disorder Questionnaire, Digit Symbol Coding, and GHQ identified as being endophenotypes for MDD and having utility in identifying genetic risk loci.

Dr David Glahn (Olin Neuropsychiatry Research Centre in the Institute of Living, Yale) discussed endophenotype discovery in SCZ in randomly ascertained pedigrees. Beginning with the MDD experience as the context, his use of a novel endophenotype ranking value (named after Irving Gottesman) was discussed. Applying this in a population sample with six cases of SCZ supplemented with 233 individuals at various levels of genetic risk, he described 10 ranked endophenotypes among neurocognitive markers and cortical surface areas.

Dr Kristen Nicodemus (University of Edinburgh) in the presentation titled 'Epistasis Increases Variation Explained in Endophenotypes above the Contribution of the Polygenic Scores' proposed the role of epistasis in variation of endophenotypes. In a study that examined ZNF804A pathway genes in 424 psychosis patients using a novel statistical model that simultaneously modeled both PRSs and epistasis, the latter was found to explain 2-3 times the variability for memory in psychosis than was explained by PRSs. The use of similar methods in cognitive endophenotypes in fragile X syndrome was introduced.

Dr Carrie Bearden (University of California, Los Angeles) discussed the results of a study from Latin America in an isolated population with 26 large families heavily loaded with BD. Neuroimaging and circadian rhythm phenotypes were characterized on the basis of high heritability and association with disease. The developmental expression of these phenotypes was studied in adolescent offspring in the families. The results suggested high rates of anxiety, inattentiveness, decreased ability to recognize facial emotions, and decreased inhibitory control in adolescents at high risk. The speaker also proposed further investigation of amygdala volume as a putative endophenotype in adolescents at risk for BD.

Dr David Porteus (University of Edinburgh) reiterated the importance of dimensional approaches such as the use of quantitative endophenotypes for examining psychiatric disorders as opposed to DSM-like categorical classificatory systems. 


\section{Eating disorders: breakthroughs and new directions in genomics and epigenomics (reported by Laura Huckins)}

Cindy Bulik (UNC) introduced the session by describing anorexia nervosa (AN), bulimia nervosa (BN), and binge-eating disorder (BED). Both $\mathrm{AN}$ and $\mathrm{BN}$ patients describe a reinforcing and anxiolytic effect from restriction and/or purging, whereas satiety is described as unpleasant. BED occurs because of a variety of emotional triggers and may be a form of impulse-control disorder. There is a large diagnostic crossover between disorders, with roughly $50 \%$ of AN patients also at some point experiencing $\mathrm{BN}$. There is also considerable crossover between BN and BED.

Stephan Ripke (Broad Institute of MIT and Harvard) described the results of a meta-analysis of two previously published AN datasets: the 2011 CHOP study (Wang et al., 2011) and the 2014 Wellcome Trust Case Control Consortium 3 (WTCCC3) study (Boraska et al., 2014). No hits were genome-wide significant. However, two regions of interest were identified, both on chromosome 12. These regions encompass SNPs that have been associated previously with type 1 diabetes, vitiligo, polycystic ovary syndrome, systemic sclerosis, and obesity-related traits.

Nadia Micali (UCL Institute of Child Health) described a gene-environment $(\mathrm{G} \times \mathrm{E})$ interaction study of eating-disorder related behaviors such as fat avoidance, fear of weight gain, bingeing, and purging. The study focused on environmental factors such as exposure to stressful life events (SLEs) and parenting abilities, and a set of previously identified candidate variants, including 5-HTTLPR, SLC6A4, rs6311 (HTR2A), rs6265 (BDNF), and variants in the MAO-A region. Participants in this study were recruited from the Avon Longitudinal Study of Parents and Children cohort (Golding et al., 2001). Roughly 10000 mothers and daughters from this study replied to questionnaires, sent when the daughters were aged 14 and 16 years. Participants were genotyped on the Illumina HumanHap550.

The experience of SLEs was found to influence bingeing and purging behaviors. Variants in MAO-A were found to influence fat avoidance and fear of weight gain. There was also a correlation between better parenting and slightly lower odds of developing these behaviors, although no correlation was found between the lowest decile of parenting ability and an increased risk. No $\mathrm{G} \times \mathrm{E}$ interactions were found.

Gerome Breen (Institute of Psychiatry, King's College, London) discussed methods to elucidate SNP heritability in AN. The heritability of AN has been estimated at 56\% (Bulik et al., 2006). AN patients often have a number of comorbid disorders, and there is evidence for shared polygenic etiology between AN and other psychiatric disorders. Breen described a method (Bulik-Sullivan et al., 2015) that examines shared heritability using LD score regression. For an SNP $\mathrm{j}$, an LD score is obtained as a sum of all the information available for SNP $\mathrm{j}$, and all SNPs in LD with SNP $\mathrm{j}$. A $\chi^{2}$ regression is then performed on that LD score. This method indicates a significant genetic correlation between AN and BMI and AN and SCZ. Correlation is also noted with other pertinent traits, including HDL, insulin resistance, TG, B-cell function, LDL, and rheumatoid arthritis (RA). 
Bulik (UNC) discussed attempts to recruit new study participants using social media. This approach allows researchers to expand the reach of their studies, to communicate more closely with parents and advocacy communities, and provide results to study participants in real time. Communicating with participants and their families enables researchers to understand and address potential concerns and questions.

Bulik (UNC) concluded the session by discussing other aspects of AN/ED research, including promising optogenetic models of binge eating in mice (Jennings et al., 2013), evidence for reduced gut microbiota diversity in AN patients, and a desire to investigate the effects of hormones, infection, stressors, and developmental stage on eating disorder development.

\section{Report of the International Society of Psychiatric Genetics genetic testing taskforce (reported by Kate Wolfe)}

Dr Francis McMahon (National Institute of Mental Health, USA) provided an introduction to the session, outlining the key measures for diagnostic genetic testing in psychiatry. Genetic tests should have analytical validity, in that they should accurately measure what they set out to measure, and clinical validity, whereby the association between the genetic variant and disease is validated. Large sample sizes and replication studies will be crucial for ensuring clinical validity. Clinical utility is another important element for consideration, particularly the potential for therapeutic interventions (http://www.cdc.gov/genomics/ gtesting/ACCE/index.htm). In 2008-2009, the ISPG taskforce broadly recommended against all genetic testing, with the exception of causal genetic and chromosomal regions (phenylketonuria, fragile X, and Huntington's disease). In 2013-2014, the taskforce reconvened to update the statement and a summary of the new recommendations has now been published on the ISPG website, with a journal article under review in the American Journal of Psychiatry (http://ispg.net/genetic-testing-statement/\#ref1). Dr McMahon introduced specialist members of the taskforce, who each presented on specific areas of the recommendations.

Dr Elliot Gershon (University of Chicago, USA) presented on the role of CNVs in psychiatric disorders. The first CNV to be characterized as a psychiatric risk locus was found on chromosome 22q11; deletions in this region were shown to increase susceptibility for SCZ (Karayiorgou et al., 1995). Gershon discussed the role of CNVs in relation to effect size versus frequency of genetic mutations, adapting the previous work of Manolio et al., 2009. Both rare and de novo CNVs can be shown to have a large effect size for psychiatric disorders. Specific risk calculations for both rare and de-novo CNVs were presented from the research of Gershon and Alliey-Rodriguez (2013). In particular, it was noted that for the rare 22q11.21 deletion, the associated risk of having SCZ, BD, or ASD is $82 \%$. The presence of a de-novo CNV anywhere in the genome has an aggregated risk of $14 \%$ for the development of a neuropsychiatric disorder, which is much higher than the population risk. Finally, Dr Gershon discussed the complex ethical implications of population-based genetic screening, particularly stigmatization and discrimination, also discussed in Gershon and Alliey-Rodriguez (2013). Dr Gershon concluded that rare disease-associated CNVs can 
increase the risk for several psychiatric disorders; they have high odds ratios and potentially have large effects on risk when involving sites contributing toward polygenic susceptibility.

Dr Daniel Mueller (University of Toronto, Canada) spoke about the use of genetic testing to guide optimal treatment in psychiatric practice. Historically, a 'trial and error' approach has guided psychiatric treatment, but the advent of pharmacogenetic markers provides the opportunity to improve physician effectiveness, reducing healthcare costs as well as the risk of serious side effects. The Mayo Clinic (USA) (under the direction of the late Dr David Mrazek) has led the field of clinical pharmocogenomic testing, utilizing a novel algorithm of drug responsiveness that is of particular benefit for patients with a poor treatment history. The initial focus was on variation in genes encoding cytochrome p450 (CYP450) enzymes (e.g. $C Y P 2 D 6$ and $C Y P 2 C 19$ ), which are involved in the metabolism of many drugs including antidepressants and antipsychotics (Hicks et al., 2013). Polymorphisms in the serotonin transporter gene and response to antidepressants (Porcelli et al., 2012) and polymorphisms in the dopamine D2 receptor and anti-psychotic response (Zhang et al., 2010) have also been explored, although more research is needed in this area. There have also been some promising results for genes involved in side effects and addiction-treatment compounds. Future research will be needed to investigate the role of epigenetics as well as genotype treatment versus treatment as usual.

Professor Marcella Rietschel (Central Institute for Mental Health, Germany) discussed the issues surrounding reporting of incidental or secondary findings, which has become a pertinent issue with the advent of exome and whole-genome sequencing. The American College of Medical Genetics and Genomics (ACMG) have produced a recommended list of genes for which incidental findings should be reported back to patients by clinicians, irrespective of the original reason for genetic testing and the age of the patient (Green et al., 2013). The ISPG taskforce concurs with this statement, but highlights that the seriousness of the disease, consequences of nondisclosure, the patient's wishes, ability to rationalize and participate in intervention, and the psychological consequences of disclosure are all important factors to consider. Although it is not yet part of clinical practice to perform exome and genome sequencing for psychiatric disorders, it is of concern that for many of the genes identified by the ACMG recommendations, we lack evidence of the predictive value of testing, genotype penetrance, spectrum of phenotypes, and efficacy of interventions in unselected populations (Burke et al., 2013). The importance of exome and genome sequencing in the research setting makes the issue of incidental and secondary findings more pertinent. Professor Rietschel went on to discuss patient opinions on genetic testing. Surveys have indicated that $97 \%$ of research participants would want to be informed of genome sequencing results that could have implications for their health and $83 \%$ of respondents would want this information irrespective of whether preventive measures or treatment are available (Bui et al., 2006). Further research by Professor Rietschel found that one-third of research participants want to know their genetic results, even if they are not relevant to the original scientific question, and some participants were even prepared to pay for feedback. Overwhelmingly, participants felt that their right to determine what they wish to know is the most important factor. It remains unclear how the ACMG recommendations should be applied in research settings and it poses a challenge in this era of rapid development; 
however, involving participants in discussions as part of the informed consent process will be key to managing these issues.

Dr Jehannine Austin (University of British Columbia, Canada) spoke about the psychological, ethical, and clinical implications of genetic testing. Dr Austin's experience is that there is a need to understand why disease has occurred, and this is no less true in the context of psychiatric disorders. The ISPG recommends that genetic risks should be communicated in a way so as to maximize understanding. Importantly, risk not only describes the probability of an event occurring, but is a broader concept integrating individual perceptions of the severity of the outcome involved. For example, it is important to frame numbers in different ways, such as reporting to parents that there is a $90 \%$ chance your child will not develop SCZ, as opposed to a $10 \%$ chance that they will. Addressing psychological issues related to changed perceptions of cause is another important role of genetic counseling (GC). Patient outcomes recorded in a psychiatric GC service were measured, and empowerment and self-efficacy were both clinically and statistically improved following GC (Inglis et al., 2015). The ISPG taskforce believed that genetic testing of minors to detect adult-onset conditions for which there are no treatment options should be discouraged, although genetic testing in those unable to consent may be appropriate under some circumstances (e.g. to identify potential CNVs where there could be relevance for associated medical issues). Dr Austin briefly discussed direct to consumer testing, provided by companies such as 23 andMe, and the vital importance of GC by appropriately qualified professionals to prevent patient misinformation about genetic test results.

The discussion was led by Professor Philip Mitchell (University of New South Wales, Australia). Professor Mitchell highlighted that it is important to consider when genetic tests should enter the clinical arena, with reference to the American Journal of Psychiatry commentary 'a bridge too far too soon' (Braff and Freeman, 2008). The analytical and clinical validity of genetic tests is particularly of concern for direct to consumer testing, with new companies emerging such as Sundance Diagnostics, who are offering a genetic test for suicidal ideation in response to SSRI treatment in depression. Another pertinent issue is whether we should now be testing routinely for CNVs in patients with SCZ (Baker et al., 2014). Professor Mitchell invited Paulina, from the Swedish National Association for Social and Mental Health, who herself has BD, to comment on the proceedings. Paulina felt that genetic tests are an important way to gain more understanding, which will in turn help to break the stigma of psychiatric disorders. It will be of utmost importance to provide support to patients with increased genetic risk through GC.

The session was opened up to questions and it was discussed that a broader aspect of clinical utility is the patient's right to know what is causing their illness, striving to place mental illness on a par with physical illness. Another concern was that more training should be provided for psychiatrists to keep pace with psychiatric genetic knowledge. The ISPG taskforce is currently in the process of developing a recommended curriculum to be included as an integral component of the psychiatric resident's curriculum. Finally, the gap between the genetically educated versus the lay person in their ability to interpret direct to consumer testing was discussed; this gap is a major issue for GC services and healthcare providers. 


\section{Systems biology approaches in psychiatric disorders (reported by Monique van der Voet)}

Dr Kasper Lage (Harvard Medical School, Massachusetts General Hospital, Broad Institute) opened the session defining systems biology as the holistic quantitative view of an entire biological system. Scientists produce data on a massive scale across many genomic platforms, which can be overwhelming. This session shows the many approaches to interpret the data and understand psychiatric disease.

Dr Lage focused on how statistics and web portals can be used to functionally interpret data in psychiatric disorders. He announced a new release of InWeb (version5), which is four times larger with 1.2 million protein-protein interactions. The DAPPLE uses random permutation to quantify whether loci in a network connect at more than a random expectation (Rossin et al., 2011). Lage reminded the audience that a network should not be the final result of a paper. It is important to validate a network to replicate a finding with an independent method. Arking et al. (2014) and Lundby et al. (2014) provide a general framework that could be translated to psychiatric disorders. Dr Shaun Purcell (Icahn School of Medicine at Mount Sinai) spoke about using biological networks to prioritize rare and common variants in SCZ-associated genes. Gene-set enrichment pathway analysis, followed by network connectivity analysis is such an approach. Using DAPPLE, the question was answered whether seed genes were more connected to each other than expected by chance and which genes are especially connected. SCZ de-novo exome sequencing trio datasets are enriched for FMRP targets (Purcell et al., 2014). GWAS regions do not have significant direct edges to this network, which is puzzling. This could be because of different kinds of genes being hit. It is not clear where this difference arises from, but may be related to de novo mutations being more constrained and brain expressed.

Professor Søren Brunak (Technical University of Denmark) spoke about integrating patient records and temporal data to understand comorbidities between psychiatric disorders and other diseases. For this, he used text mining of data from an electronic health registry covering the entire population of Denmark (Jensen et al., 2014). The analysis shows trajectories of disease development, disease-disease correlation, and adverse drug reactions. Initially, it was surprising to observe an epidemiological relationship between gout and cardiovascular disease, but other studies support this association. The population-wide disease trajectory approach has the power to uncover new disease-disease correlations, which will be a powerful approach to understand comorbidities between psychiatric disorders and other diseases.

Dr Iiris Hovatta (University of Helsinki) spoke about animal models to understand the genetic basis of anxiety disorders (Sokolowska and Hovatta, 2013). She combines mouse and human genetic approaches to identify genes that regulate anxiety. Known brain anxiety circuits were analyzed in an innate anxiety mouse model. RNAseq and miRNAseq identified gene pathways involved in anxiety-like behavior in the mouse, where NF- $\kappa \mathrm{B}$ acts like a hub. $\mathrm{G} \times \mathrm{E}$ interaction was analyzed in a social defeat mouse model that causes long-term changes of 232 genes in the brain. These new genes are good candidates for genetic studies in humans. 


\section{Copy number variations in adults with neurodevelopmental disorders (reported by Samuel J.R.A. Chawner)}

Professor Griet Van Buggenhout (KU Leuven - University of Leuven, Belgium) reported a high prevalence of pathogenic CNVs in adults with a dual diagnosis of intellectual disability (ID) and neuropsychiatric disorder. It was highlighted that there is a lack of studies examining the neurodevelopmental phenotype of adults with pathogenic CNVs. Results were presented from an international collaborative effort between University College London, Universitat Autonòma de Barcelona and KU Leuven - University of Leuven. Five hundred adults with dual diagnosis were recruited through psychiatric units for ID and also genetic clinics within psychiatric hospitals. Microarray analysis was carried out on all individuals and it was found that $36.4 \%$ of adults with dual diagnosis had a likely pathogenic CNV and the most frequent CNVs were at the following loci: 2p16.3, 3q29, 7q11.23, 15q11.2, 15q13, 16p11.2, 17p11.2, 22q11.21, and 22q11.33.

Dr Ramon Novell (Institut Assistencia Sanitaria, Spain) presented case studies from three families with deletion of the 2p16.3 region that includes the NRXN1 gene. The psychiatric and cognitive phenotype of probands and family carriers was variable and included SCZ, bipolar, autism, ADHD, and generalized anxiety disorder, and there were carriers with no diagnosis. All three probands fulfilled the criteria for mild ID and their IQ ranged from 53 to 65. Intellectual functioning in family carriers ranged from mild ID to being within the normal range, with IQ scores ranging from 69 to 91. Both probands and carriers showed impairments in executive function. The phenotype of carriers suggests that the $2 \mathrm{p} 16.3$ deletion has variable expressivity instead of incomplete penetrance.

Professor Sébastien Jacquemont (Université de Lausanne, Switzerland; Université de Montréal, Canada) spoke about the contrasting phenotype of reciprocal rearrangements at the 16p11.2 locus, a region implicated in head size, obesity, and neurodevelopmental disorder (Shinawi et al., 2010; Jacquemont et al., 2011). Fourteen individuals with the deletion of the 16p11.2 region, 17 with duplication of the region, and 23 intrafamilial controls underwent neuroimaging. An effect of genomic copy number was found for wholebrain volume, both gray and white matter volume, cortical surface area, and for the volume of regions involved in the reward pathway (after correction for whole-brain volume). The pattern of brain anatomy changes in carriers spatially overlapped with established structural abnormalities in autism and SCZ. Using measures of peripheral mRNA levels, analysis confirmed the results obtained with genomic copy number. The findings highlight endophenotypes that mediate the effect of the 16p11.2 locus on behavior.

Professor Annick Vogels (KU Leuven - University of Leuven, Belgium) reported the phenotype of individuals with 22q11.2 deletion syndrome (22q11.2DS) [recognized as a genetic model for SCZ (Schneider et al., 2014)] who were diagnosed in adulthood. A retrospective study examined clinical records of 65 individuals with 22q11.2DS diagnosed as adults. They were recruited through a genetics clinic (referred to the outpatient clinic), a residential unit for ID (actively recruited), and a psychiatric unit for adults with ID (actively recruited). There were high rates of psychiatric disorder including $45 \%$ with SCZ spectrum disorder, $45 \%$ with personality disorder, and $10 \%$ with mood disorder. Physical features such as facial or cardiac abnormalities were rare. The results emphasize the need to consider 
the diagnosis of 22q11.2DS in adults with ID and psychiatric disorder even in the absence of obvious physical features.

In the discussion, the role of the psychiatrist in managing patients with pathogenic CNVs was considered and there was consensus that intervention during childhood was important, given the high rates of psychiatric disorder in adulthood. However, the panel mentioned that there is a current lack of guidelines for appropriate intervention strategies in these clinical populations. The ethics of prenatal testing of pathogenic CNVs were discussed and the panel believed that such testing should be accompanied by specialized GC.

\section{Immunomics: exploring new territory in schizophrenia (reported by Luca Pagliaroli)}

Jennie Pougeut (Centre for Addiction and Mental Health) focused on the immune hypothesis of SCZ. From the GWAS-SCZ sample with 9394 cases and 12462 controls, 927 'immune' genes and 18165 immune SNPS tagging 814 genes were identified. However, the only genome-wide significant signals were detected in PGG1 and PGG2. No improvement in the analysis of the data has been found using hypothesis-driven GWAS. This approach prioritizes the SNPs in immune-nonimmune genes to calculate the data-driven weighting for immune-nonimmune data and $q$-values for each SNP. Nevertheless, prioritization does not improve the discovery of immune loci in SCZ. In conclusion, the immune system does not seem to be the major etiological pathway in $\mathrm{SCZ}$ and it may be involved downstream rather than at the beginning of the disease.

Dr Wray (the University of Queensland) spoke about the epidemiological puzzle of RA and SCZ. This puzzle is complicated as smoking is a major risk factor for RA and smoking rates are high in those with SCZ. The investigation of the genetic relationship is not possible using the traditional epidemiological approaches because of the following reasons: the need for very large samples, complications of age of onset and sex, and risk of incurring both SCZ and RA by chance $0.01 \%$.

The main messages of this talk were (i) the need to intensively analyze the available data in order to generate hypotheses and (ii) the necessity of further analysis for a better understanding of this complex puzzle.

Dr McCarroll (Stanley Center for Psychiatric Research, Broad Institute of MIT and Harvard) illustrated his project aiming to understand those forms of structural polymorphism that are not present in HapMap or 1000 genome, not visible to array-based copy number analysis, and not tagged by di-allelic SNPs. In particular, attention was focused on HLA-C4, which is an interesting candidate in SCZ and exists in long (L) or short (S) form and in an A or a B variant. He was able to show that AL-BS segregates into five different haplotypes, AL-BL segregates into three different haplotypes, and AL-AL segregates into two different haplotypes. He further suggested an association between allelic haplotypes of the $C 4$ structure and SCZ according to the following pattern: 'the more the brain $C 4 A$ expression is associated to a structure the more SCZ risk is associated with that structure'.

Dr Yolken (Johns Hopkins) talked about the microbiome. The assumption is that every individual has a unique set of microorganisms at mucosal sites that are larger and more 
complex than the human one and is partially inherited from the mother and partially acquired from the environment. A high-throughput sequencing of throat swab samples, $n=$ 41 with SCZ and $n=32$ without psychiatric disorders, showed that the composition of the control microbiome is different from that of SCZ patients. Although the two groups do not differ in age, sex, race, cigarette smoking, and oral hygiene, they differ in the rate of deficit syndrome and evidence of intestinal inflammation. Further screening of 16 patients and 16 control participants showed differences in viruses between them, which might be suggesting that some kinds of viruses may be protective whereas others could be associated with illness.

In conclusion, the study of the microbiome is an extremely important new approach to the pathophysiology, and potentially the treatment, of psychiatric disorders.

\section{The effect of psychosis risk genes across the phenotypic spectrum (reported by Maria Tropeano)}

Dr Thomas Hyde (Lieber Institute for Brain Development, USA) discussed the importance of the study of human postmortem brain to analyze the effects of the SCZ-associated genetic variants on a molecular level. For a number of genes, studying the expression of the fulllength transcript does not fully explain how the risk allele might alter gene expression, either in the fetus or in the adult. Straub et al. (2007) identified a SNP in the promoter region of the $G A D 1$ gene, associated with the risk of SCZ and with decreased expression of the GABA synthetic enzyme GAD67 in SCZ postmortem brain. Carrying out gene expression analyses in postmortem human brain, Dr Hyde has identified a new transcript derived from the GAD1 gene, named GAD25, which encodes a truncated protein that lacks the GABA synthetic domain and is highly expressed in the developing fetal brain. SCZ cases homozygous for the $G A D 1$ risk allele have shown an increase in the expression of GAD25 in the hippocampus, which lend support to the hypothesis that SCZ could be caused by anomalies in the normal trajectory of brain maturation. A key step toward the understanding of the molecular mechanisms of genetic risk relies upon defining the family of transcripts derived from each risk gene.

Dr Ole Andreassen (University of Oslo, Norway) presented his work investigating the functional consequences of the newly identified SCZ risk genes in vivo using brain imaging technology and deep phenotyping in SCZ patients. He showed examples of SCZ GWASs hits that have been found to be associated with functional brain abnormalities, such as common genetic variants in the MHC, a region repeatedly implicated in SCZ, that have been associated with larger ventricular volumes in SCZ patients (Agartz et al., 2011). He also described a novel Bayesian statistical framework, developed recently by his group with the aim of investigating the complex effect of a series of SCZ-associated risk variants (PRS) on neuroimaging phenotypes in SCZ patients; his preliminary findings showed a strong effect of the SCZ PRS from the PGC on the dorsolateral prefrontal cortex (DLPFC) of SCZ patients from a GWAS of 3000 MRI brain scans. He concluded by highlighting the need for novel statistical methods and for larger sample sizes to increase the statistical power of imaging genomics studies.

Dr Anil Malhotra (Zucker Hillside Hospital, USA) discussed the potential genetic overlap between general cognitive ability and SCZ. Generalized deficits in cognitive ability 
represent a core component of SCZ; however, to date, there is no molecular evidence in support of this overlap. He presented data from a large-scale GWAS, meta-analysis of general cognitive ability (g) from the Cognitive Genomics consorTium (COGENT), including $\sim 5000$ healthy volunteers from nine sites across seven countries. Polygenic SNP scores from the cognitive GWAS meta-analysis were used to test whether alleles associated with reduced $\mathrm{g}$ also increased the risk for $\mathrm{SCZ}$ in four $\mathrm{SCZ}$ case-control cohorts. As predicted, SCZ cases had significantly lower cognitive polygenic scores compared with the controls. In parallel, PRSs for SCZ were associated with lower $\mathrm{g}$ in the COGENT samples (Lencz et al., 2014). Finally, Dr Malhotra also presented some preliminary findings from the COGENT phase 2 study, including data from 24544 healthy volunteers. In the new sample, he identified an SNP on chromosome 6 (rs1906252) significantly associated with 'g'; he noted that two proxies of this SNP have recently been associated with educational attainment (EA) (rs9320913, $r^{2}=0.91$; Rietveld et al., 2013) and with increased risk for BD (rs12202969, $r^{2}=1$; Mühleisen et al., 2014) in two other independent GWAS.

Dr Aristotle Voineskos (University of Toronto, Canada) spoke on the use of additive risk modeling (Hill et al., 2008) to achieve a more comprehensive neurobiological understanding of phenotypic variability among individuals with SCZ. He presented data from a sample of 198 SCZ patients and healthy controls, in which he investigated the association between brain structure and cognitive performance and an additive genetic model, including three SCZ-associated risk variants from GWASs (MIR137, CACNA1C, ZNF804A) and two risk variants from genes with well-established effects on brain structure and function (GAD1, $B D N F)$. He found that the additive SCZ risk score significantly predicted white matter integrity throughout the brain and was associated with increased cortical thickness and poor cognitive performance (particularly in verbal fluency and motor functioning) in patients with SCZ. In conclusion, he emphasized the need for longitudinal studies to confirm these findings and also highlighted the potential importance of additive genetic risk models in the prediction of disease progression in SCZ patients at early stages of the disease, and to inform treatment decisions.

\section{Neuropsychiatric genetic research in Latin America (reported by Antonio Pardiñas)}

Dr Henriette Raventos (Universidad de Costa Rica) started the session by commenting on the advantages of psychiatric and genetic research in her home country. First of all, Costa Rica has a wealth of ecclesiastical records on families from the 18th century on and detailed genealogical records since 1890. These have been incorporated into a centralized universal healthcare system, which allows medical researchers to access this data. Besides this, families are supportive of genetic research and many different population groups exist as isolates separated by mountains and valleys. All this together has proven powerful to carry out linkage studies using population registries, which have shown the molecular basis of several forms of inherited deafness and BD (Leon et al., 1992; Mcinnes et al., 2001). In recent years, focus has shifted toward admixture studies, which have shown the presence in the Costa Rican population of at least four ancestry components (European, African, Asian, and Amerindian), information that can be used for association studies and might highlight new risk or protective alleles for medical conditions (Segura-Wang et al., 2010; CamposSánchez et al., 2013). These results, along with the latest studies on psychiatric disorders 
(Gonzalez et al., 2013), have been influential for public policy within the country. Currently, the focus is to develop and complete a large database of clinical and genetic information, which will store around 10000 samples. Also, functional studies on transcription factors have been published (Marballi et al., 2014), studies on a microRNA role in psychiatric disorders are ongoing, and a laboratory for the study of the zebrafish as an animal model is starting its course.

Professor Homero Vallada (University of Sao Paulo Medical School) spoke about Brazil, a country that houses $50 \%$ of the South American population and $80 \%$ of its Research \& Development budget. Brazil is noteworthy for a huge population expansion since the start of the 19th century and for having been the destination of $40 \%$ of the Trans-Atlantic slave trade. This makes it an admixture melting pot, with patterns that reflect the original colonization of the territory (Salzano and Sans, 2014; Magalhaes Da Silva et al., 2015). Although the first genetic studies in Brazil started in the 1980s, interest in genetics increased after the publication of the first 'Brazilian Genome Project', the plant pathogen Xyllela fastidiosa (Simpson et al., 2000). The fast subsequent development provided the necessary infrastructure to carry out the Baependi Heart Study (De Oliveira et al., 2008), and start several major projects on addiction (Lobo et al., 2014), cognition (Santos et al., 2014), and pharmacogenomics (Suarez-Kurtz et al., 2014).

Dr Humberto Nicolini (National Institute of Genomic Medicine INMUGEN) introduced the latest research developments in psychiatric genetics in Mexico. Several of them built on the latest knowledge on BD, with the aim of explaining mood changes (Gonzalez et al., 2014). The large familiar structure present in the country is extremely useful for research as hundreds of extended families with at least two affected sib-pairs have been recruited in the past (Glahn et al., 2010), and these recruitments are still in progress. Also, there is the possibility of examining the effects that nutrition and quality-of-life changes over the last century might have had on the physical and mental well-being of the Mexican population using molecular and genetic techniques (Hare et al., 2010; Estrada et al., 2014).

Professor Carlos Lopez-Jaramillo (University of Antioquia) finished the session. He spoke in depth on the research on Colombian population isolates, such as the Paisas (Bedoya et al., 2006). This population was founded in the 17th century by admixture between Spanish men and Native American women, and has been studied extensively because of its increased prevalence of psychiatric disorders such as ADHD, PD, and BD (Pineda et al., 2011; VelezPardo et al., 2015), as well as suicidality, which is 10 times higher than that in the general Colombian population (Alzate and Benitez, 2011). One of the best-developed lines of research in Colombia are pedigree studies as many extended families with 40-50 members can be recruited, which enables investigators to carry out detailed studies even at the phenotype level (Fears et al., 2014). Recent efforts to phenotype these samples with a higher resolution include using actigraphy to study circadian rhythm patterns and substance tests to measure abuse. Also, a new study is ongoing in the small population of Aranzazu, an isolated village of 12000 inhabitants in which BD type 1 has a total prevalence of 6-8\%, and is currently the fourth most common cause of hospitalization. 


\section{Genetics and aggression: the Aggressotype project (reported by Luca Pagliaroli)}

Dr Franke (Department of Human Genetics and Psychiatry, Donder's Institute of Brain, Cognition and Behaviour, Radboud University Medical Center) presented an overview of the Aggressotype project. As part of the EU FP7 program, the international consortium Aggressotype was founded in 2013 and focused on aggression and impulsive behavior in ADHD and conduct disorders. This project involves preclinical research at different levels: from genetics, to cells, to brain function, and eventually to behavior. The following areas are under investigation: (i) epigenetics studies of animal models to find biomarkers; (ii) imaging studies of animal models; (iii) alternative and classical animal model approaches suitable for behavioral studies and high-throughput screening; and(iv) NeuroIMAGE projects to study human behavior.

In conclusion, different levels of research are the key to understanding pathologic aggression. To achieve this goal, 24 academic partners and 4 industries have joined forces in this project.

Dr Norton (University of Leicester) talked about the use of zebrafish as an animal model for aggression. This species seems to be an excellent model because of the possibility of having stable mutant lines. The grade of aggression is easily measured either through dyadic fights between two fish or by looking at the interaction with a mirror. Spiegeldaino $(s p d)$ is a zebrafish fgfrla mutant that shows increase in aggression, boldness, and exploration compared to the wild type. Global analysis of 40 neurotransmitters signaling in $s p d$ showed a $15 \%$ increase in $h n m t$ expression and a phenotype rescue after treatment with tacrine hydrochloride $(1 \mathrm{mg} / \mathrm{ml})$. A new screening for novel aggression therapeutics led to the identification of five novel compounds that reduce aggression (data on adult fish). The aggression level during ontogeny after days 12,21 , and 25 of treatment showed that at 25 days, the majority of fish have aggressive interactions with the mirror. Different studies showed that fluoxetine hydrochloride reduces aggression in adults, whereas WAY100 635 exerts the opposite effect. The aggression phenotype has also been addressed in juvenile fish with similar results; furthermore, the effect of methylphenidate and risperidone has been investigated, showing a reduction of juvenile aggression. Screening for novel drugs is expected to start in January 2015.

Dr Glennon's (Rabdoud University Medical Center) speech was focused on the use of an animal model of aggression: the BALB/cJ. Its goal is to investigate whether the aggressive traits of rodent models are associated with changes in structural and functional brain connectivity, neurochemistry, and epigenetics. The BALB/cJ mouse strain has a $\mathrm{C} 1473 \mathrm{G}$ polymorphism in the tryptophan hydroxylase-2 gene. This mouse is more aggressive and shows an anxious phenotype with more evidence for a hypoarousal state after a fight than control. FA MRI-DTI studies indicated altered corticostriatal connectivity in the anterior cingulate cortex and the dorsal medial striatum. Mice have also been tested using a novel virtual reality setup that enables the analyses of behaviors relevant to aggression combined with optogenetics and tetrode recording. The paper of Márquez et al. (2013) suggests that early life stress is linked to aggression and leads to MRI changes in the right amygdala. Further investigations should involve mapping of genetic and epigenetic markers to neuronal networks of aggression. 
Dr Zayats (Bergen University) talked about her project on 'epigenetics in mental disorders, maternal and parent of origin effects' and the need for investigation on ADHD to understand the origin of aggression. It is important to gain knowledge of genetics as ADHD is the most heritable childhood onset neuropsychiatric condition (30-90\%). It has been reported that an asymmetry in parental transmission to ADHD affected offspring implicated several genes ( SNAP25, HTRB1, SLC6A4, SLC6A2, TPH2, DRD4, DRD5, DDC, BDNF). Genome-wide examination of imprinting in ADHD suggested the implication of FAS and PDLIM1 genes. However, no genome-wide significant findings were reported on GWAS results and candidate genes studies failed to replicate. The use of a multinomial regression model to investigate the parent of origin effect enables the differentiation of imprinting and maternal effects. Its application to the GWAS of Neale et al. (2008) identified rs 96766447 with a $P$ value of $6.72 \mathrm{E}-07$ (maternal effect $P=0.0046$ and transmission asymmetry $P=0.013$ ). Furthermore, from a study of Wang et al. (2011), it has been possible to identify rs7790549 with an overall P-value of 6.70E - 06 (maternal effect $P=9.82 \mathrm{E}-06$ and transmission asymmetry $P=1.39 \mathrm{E}-04$ ). Therefore, the application of a multivariate approach is very important to detect ADHD candidate genes.

\section{Toward translational psychiatry: from genomic discoveries to prediction of treatment response (reported by Erik Loken)}

Dr Yu-Lin Chao (Tzu Chi General Hospital, Taiwan) presented his work on inversely regulated microRNAs in a mouse model of psychosis treated with electro-convulsive shock (ECS). Dr Chao looked at the microRNA of mouse hippocampus after methamphetamine and ECS. The hypothesis tested was whether specific microRNAs modulate response to ECS. Dr Chao found that prepulse inhibition (PPI) deficits and abnormalities in the novel object recognition test induced by chronic methamphetamine administration were improved after repeated ECS. Conditioned place preference deficits showed no sustained treatment effect with ECS. On analyzing microRNAs, Dr Chao found that more microRNAs were significantly downregulated after ECS. A pathway analysis of the six candidate microRNAs, $652,328,138,339-5 p, 126-5 p$, and 203 , found that they were involved in axon guidance. MiR-328 was successfully overexpressed; after injection into the hippocampus, the overexpressed miR-328 was associated with further impaired PPI on methamphetamine administration. Downregulation of miR-328 reduced the PPI deficit. A question was asked about whether any gene targets for these microRNAs overlapped with SCZ risk genes, and Dr Chao responded that they had found a very large overlap with both SCZ and BD risk genes.

Dr Wei J. Chen (College of Public Health, National Taiwan University, Taiwan) reported on blood-based microRNA expression aberration in SCZ. Dr Chen studied 48 SCZ and 37 controls samples and looked at their peripheral blood miR-34a, miR-449a, miR-564, miR-432, miR-548d, miR-572, and miR-652 microRNA levels to observe whether they changed from acute admission to partial remission in inpatients with $\mathrm{SCZ}$, and also whether they were associated with brain structure differences in patients with SCZ. She found a persistence of aberrant expression of the seven microRNAs in peripheral blood from admission to discharge, indicating that these microRNAs were not useful for treatment 
outcome. Dr Chen plans to investigate peripheral microRNA expression further as a biomarker for SCZ.

Dr Ya-Chin Lee (College of Public Health, National Taiwan University, Taiwan) presented work on the identification of novel biomarkers for manic episodes. He used whole-genome transcriptome analysis with the specific aim of finding changes to the transcriptome in response to mania, which would hopefully be useful as a new set of biomarkers. Dr Lee studied six BD patients and using the Affymetrix Human Transcriptome Array 2.0, used paired $t$-tests to compare remission expression versus manic-episode expression. He used a threshold P-value of 0.01 and at least a 1.3-fold change to select 74 annotated transcripts fulfilling the criteria. Only five of the transcripts were coding genes. There were 32 lncRNAs, 15 snRNA, snoRNa, and rRNAs, and 22 miscRNAs. Dr Lee cited research that implicates a lncRNA, Gomafu, as downregulated in SCZ postmortem superior temporal gyrus (Barry et al., 2014). Dr Lee used STRING in a protein-protein interaction analysis of transcriptome data that implicated immune-associated pathways.

Dr Po-See Chen (National Cheng Kung University Medical College, Taiwan) presented work investigating treatment response to antidepressants in patients with MDD. Only 40$50 \%$ of patients respond to anti-depressants, which can be predicted by demographics, illness features, environmental factors, and genetic factors. He used artificial neural networks to analyze the effect of the pharmacodynamics genes $G N B 3$ and $B D N F$ and the pharmacokinetic genes $2 C 19^{*} 3$ and $A B C B 1$ and $C 2677 A$ on the treatment response to fluoxetine in 73 patients and venlafaxine in 76 patients. In addition to these genetic factors, he examined the biomarker $h s-C R P$ and social support scales. Using $80 \%$ of patients to train and $20 \%$ to test the artificial neural network, Dr Chen found the artificial neural network to be more useful to predict response to antidepressants than linear regression techniques. $B D N F, C R P$, and social support were significant in the analysis.

\section{The interplay between genetics and early trauma in severe mental disorders (reported by Monica Aas)}

Marco Riva (Department of Pharmacological and Biomolecular Sciences, University of Milan, Italy) presented his recent work investigating the role of epigenetics in early stress and long-term psychopathology. Riva described the use of a rat prenatal stress (PNS) model to investigate molecular and functional changes that could contribute toward the development or the maintenance of a phenotype on the basis of early life adversity. This was done by examining changes in the expression of neurotrophins and epigenetic regulators as well as changes in prefrontal methylation of gene promoters relevant for neuronal function using a cross-species approach. At the molecular level, PNS rats showed a region-specific and time-specific reduction in the expression of the neurotrophin BDNF, a marker of neuronal plasticity that plays an important role in mood and cognitive function. Professor Riva also presented data showing that exposure to PNS produces significant changes in the expression of several epigenetic regulators, including DNMT1, Gadd45 $\beta$ as well as HDACs.

Annamaria Cattaneo (King's College of London, Institute of Psychiatry, UK) presented recent work investigating blood transcriptomics as a tool to identify the long-lasting effects of childhood trauma on psychopathology. Cattaneo reported that alterations in the mRNA 
levels of genes involved in the stress response are particularly related to changes in the mineralcorticoid receptor, the GR, and the serum glucocorticoid kinase (SGK1), which is specifically activated by glucocorticoids. An additive effect of depression and trauma on modulation of SGK1 mRNA levels was observed. Similarly, Dr Cattaneo showed that SGK1 mRNA levels were increased in the hippocampus of adult rats exposed to prenatal stress. These findings support a long-lasting effect of prenatal stress on SGK1 levels.

Bruno Etain (APHP - Inserm U955 and Fondation FondaMental, Creteil, France) presented his recent work on the topic of childhood trauma and BDs - severe clinical expression and moderation by genetic factors. The talk discussed how different types of childhood trauma influenced clinical expression and severity indicators in 587 patients with BDs, including interaction between early trauma and known variants of the serotonin transporter gene. Emotional and sexual abuse was associated with a more severe expression of the disorder such as an earlier age at onset (AAO), increase in suicide attempts, more rapid cycling, and greater proneness to depression. There was an additional effect of the 5-HTTLPR genotype and trauma on time to onset of BD. Finally, a history of childhood trauma, particularly emotional abuse, was associated with a significantly higher score on the Affective Lability Scale in two independent samples of patients with BDs (collected in France and Norway).

\section{Utilizing family-based information in genetic research (reported by Suhas Ganesha)}

This session was chaired by Dr Pippa Thompson (Institute of Genetics and Molecular Medicine, University of Edinburgh). She introduced the session by citing the importance of high-frequency rare variants in the genetic etiology of neuropsychiatric disorders and the shortcomings of routine case-control and cross-sectional approaches for identifying them.

Dr Lan Xiong (University of Montreal) presented on the advantages of using family-based cohorts to study neuropsychiatric disorders. In whole-exome and genome sequencing in Canadian BD family-based studies, an HTR3A missense variant was reported to be highly associated with lithium response. In 31 inbred Iranian bipolar pedigrees, 600 shared/novel variants were identified. Thirteen genes were found to have more than one novel/rare variant in more than one family. Similarly, large consanguineous pedigrees from Pakistan with SCZ cases were presented, including exome sequencing data from a family of 13 affected individuals. Dr Simone de Jong (King's College London) presented linkage and sequencing data in a large Brazilian bipolar family with 111 affected cases. Of the samples obtained from $300+$ members in a family, $30 \%$ had from a mood disorder. BD showed anticipation across the four generations studied. Genome-wide significant linkage, allowing for multiple phenotype definitions, was identified for 2p23.1-p22.3 (LOD = 3.83) for all mood disorders, 3 p23-p24.1 (LOD = 4.18) for narrow bipolar 1, and both 11p14 (LOD = 4.49) and 12q24.22-q24.32 (LOD = 4.74) for depression. Fine mapping of eight linkage peaks by exome sequencing and variant filtering identified mutations in ATR5, DNHD1, and DCHS1 genes for a depression phenotype. Similarly, fine mapping of all affected mood disorder cases identified an association with the ITGA1 gene and the FAM98 gene. These genes are known to be involved in cell signaling, adhesion, translation, and neurogenesis.

Dr John Blangero (Texas Biomedical Research Institute) discussed the utility of studying rare variants using randomly ascertained pedigree studies, and thus to characterize their 
biological effects. The San Antonio Family Study/Genetics of Brain Structure and Function Study was presented. Caudate volume and frontal cortical thickness were presented as prototype heritable traits influenced by rare variants including a novel variant on chromosome 19 and a variant on $D E G S 1$ involved in the ceramide synthesis pathway. The utility of endophenotype ranking values with cortical surface area as an endophenotype was discussed. In an interesting attempt at Neanderthal genomic/phenomic reconstruction, evidence for reduced connectivity, impaired memory function, and reduced Broca's speech areas was shown.

In the final presentation, Dr Pippa Thomson (Institute of Genetics and Molecular Medicine) presented a study to identify rare variants using exome sequencing data from 277 cases from 44 families and 148 unrelated individuals of familial recurrent MDD drawn from the Generation Scotland: Scottish Family Health Study. Linkage analysis identified two peaks: one at the tip of chromosome 12 within a microdeletion region for neurodevelopmental delay and the other peak was noted to overlap the centromeric region of chromosome 18 , which was also additionally linked to depression endophenotypes of AAO, memory, and cognitive ability. NINJ2 and ROCK1 on chromosome 12 and chromosome 18, respectively, showed an increased frequency of rare variants implicated in neurite outgrowth and response to nerve injury.

Dr Andrew McIntosh led the discussion and stressed the reemerging importance of familybased studies in the identification of rare variants in psychiatric disorders.

\section{Exploring the evidence that postpartum depression is a more homogeneous biological subtype of major depression (major depressive disorder) (reported by Martin Tesli)}

Dr Patrick Sullivan (University of North Carolina, USA) introduced this session by comparing the success of the PGC with SCZ with the lack of identification of new risk loci for MDD. He discusses potential causes for this lack of findings, including low statistical power, hidden $\mathrm{G} \times \mathrm{E}$ interactions, and phenotypic heterogeneity. He also suggested that postpartum depression (PPD) may be more homogenous and heritable than the broad category of MDD.

Alexander Viktorin (Karolinska Institutet, Stockholm, Sweden) followed up on this discussion by arguing that perinatal depression (PND) is probably neither totally overlapping nor totally independent from MDD, but somewhere in between. He moved on to presenting his own research on the heritability of PND and genetic overlap with nonperinatal major depressive disorder (NPMDD), a major depressive episode at any other time of life. For this purpose, the authors investigated heritability in 3427 parous twin women with a classical twin-model using a lifetime version of the Edinburgh Postnatal Depression Scale and estimated narrow sense heritability of PND at 54\%. Further, using data from Swedish national registers, they divided MDD into PND and NPMDD and investigated heritability in over 580000 parous female siblings. Using the register data, they estimated the heritability of PND at $44 \%$ and the heritability of NP-MDD at $32 \%$, while also showing that one-third of the genetic variation in PND was unique for the disorder and not seen in NP-MDD, supporting the hypothesis that depression during the perinatal period is influenced by genes to a greater extent than depression at other times of life. 
Dr Naomi Wray (University of Queensland, Brisbane, Australia) pointed out the dilemma in MDD between sample size on the one hand and phenotypic homogeneity on the other. To test the hypothesis of PPD as a genetically more homogeneous category than MDD, Wray and colleagues compared BD PRS between PPD and MDD patients in two independent populations: one from Australia (1450 MDD cases including 484 PPD, 1703 controls) and the other from the Netherlands (1699 MDD cases including 208 PPD, 1765 controls). Interestingly, they found that BD PRS explained more of the phenotypic variance for PPD $\left(r^{2}>1.1 \%, \mathrm{P}<0.008\right)$ than for MDD $\left(r^{2}=0.06 \%, \mathrm{P}=0.08\right)$, suggesting that $\mathrm{PPD}$ and $\mathrm{BD}$ share more genetic risk than MDD and BD (Byrne et al., 2014).

Dr Samantha Meltzer-Brody (University of North Carolina, USA) discussed the possibility of identification of more homogeneous subtypes of PPD by latent class analysis. Data were described from the international perinatal psychiatry consortium, PACT (Postpartum Depression: Action towards Causes and Treatment), comprising 24 institutions in seven countries, and 27776 patient records submitted with phenotypic data. Using the latent class analysis approach, the researchers found that a three-class solution yielded the best fit of the phenotypic data. Severity, timing of onset, comorbid anxiety, and suicidal ideation were the most distinguishing characteristics. Dr Meltzer-Brody concluded that precise phenotyping is important for further exploration of underlying biomarkers and etiological factors in PPD.

Dr Divya Mehta (the University of Queensland, Australia) spoke on the possibility of using gene expression profiles from women in the third trimester as a biomarker for PPD. In a sample of 29 PPD cases and 40 controls, 116 gene transcripts were expressed differentially (corrected $\mathrm{P}<0.01$ ), distinguishing cases from controls with $88 \%$ accuracy. Further, functional annotation implied a role of estrogen signaling (Mehta et al., 2014). Dr Mehta also highlighted the need for large international collaboration projects to achieve statistical power for detecting robust and reproducible biomarkers in PPD.

The following issues were addressed during the panel discussion: (i) the relation between PPD, BD, MDD, and postpartum psychosis. (The PACT study will also investigate postpartum psychosis.) (ii) The heterogeneity of PPD (e.g. there is evidence for hormonal trigger sensitivity in PPD). Environmental risk may also play a role.(iii) How to get the 'noise' out of PPD, while increasing sample size. (iv) The possibility of recruiting PPD cases through social media and creating an online community.

\section{Genetics of lithium response in bipolar disorder (reported by Jie Song)}

Dr John Kelsoe (University of California, San Diego School of Medicine, USA) introduced the session by stressing that response to lithium in BD may distinguish mechanistically different forms of illness. The identification of genetic variants associated with good lithium response may therefore yield a way to dissect BD into less heterogeneous parts.

Dr Urs Heilbronner (Ludwig-Maximilian University Munich, Germany) presented the latest GWAS results of the International Consortium on Lithium Genetics (ConLiGen) in 2587 patients. Two definitions of lithium response (dichotomous and continuous) were used on the basis of the 'Retrospective Criteria of Long-Term Treatment Response in Research Subjects with Bipolar Disorder' scale (informally referred to as 'Alda Scale') (Manchia et 
al., 2013). The inter-rater agreement and reliability were moderate to substantial. ConLiGen carried out two nonoverlapping GWASs on 1186 and 1401 patients, respectively. A metaanalysis combining them yielded one directly genotyped SNP, rs74795342, along with three imputed SNPs showing genome-wide significant associations with lithium response using the continuous response definition. These four SNPs in high LD are located in a long intergenic noncoding RNA (lincRNA) region on chromosome 21. Recent studies suggest the widespread roles of lincRNAs in gene regulation and other cellular processes. Further studies might provide insights into the biological mechanism of lithium's action. He also mentioned that the associations showed robust association in a subgroup of the sample diagnosed with bipolar I disorder. The dichotomous definition of lithium response did not yield a genomewide significant result. Using this phenotype, the result with the lowest $P$ value was also located in a lincRNA region on chromosome 14.

Dr Martin Alda (Dalhousie University, Canada) summarized work in the past two decades investigating responders to lithium as a distinct subgroup of BD. Applying the five-point criteria of diagnostic validity (Robins and Guze, 1970) to BD responsive to long-term lithium treatment, they identified that patients responsive to lithium showed specific clinical features (Grof et al., 1993; Calabrese et al., 1996; Greil et al., 1997; Baldessarini et al., 2000; Bowden, 2001; Passmore et al., 2003). In addition, response to lithium is a longitudinally stable and familial characteristic (Grof et al., 2002; Berghofer et al., 2008). Both clinical observational and neurobiological studies (Passmore et al., 2003; Kruger et al., 2006) supported the view of lithium responders as a distinct group that differs from responders to other mood stabilizers. In the review of genetic findings, their group previously showed CREB family of genes (Mamdani et al., 2008; Alda et al., 2013) and synapse-related genes (Lopez de Lara et al., 2010; Cruceanu et al., 2012) associated with lithium response. Moreover, Alda presented their newest genome sequencing results. Wholeexome sequencing and whole-genome sequencing were performed combining samples of families and unrelated probands characterized for their response to long-term treatment. The most interesting findings included rare variants in $H T R 3 A$, HTR $3 B$, and $H T R 3 C$ genes in single families and enrichment of G-protein-coupled receptor gene deleterious variants in lithium-responsive families. He finally pointed out that specific genetic variants associated with lithium response may not be directly useful because of their modest effect or rare frequency, but they can provide insight into the mechanisms of the illness and the medication.

Dr Sarah E. Bergen (Karolinska Institutet, Sweden) described GWAS results involving both self-reported measures (in 2698 bipolar patients) and objective clinical records (in 1211 bipolar patients) of lithium response in cases and 8899 healthy controls. When comparing lithium responders and nonresponders, the genome-wide association meta-analysis combining Swedish and UK samples together showed no genome-wide significant associations. In addition, they carried out genome-wide association analyses comparing the lithium-responsive subgroup of patients with controls without a history of psychiatric disorders. The meta-analyses yielded two SNPs, rs146727601 (located in the PTS gene) and rs116323614 (located in the SESTD1 gene), with genomewide significant associations for the objective response group. Although the confirmation through direct genotyping of these imputed markers and replication in larger samples is warranted, their findings again 
suggested that lithium-responsive bipolar patients may define a more etiologically homogeneous patient population.

Dr John Kelsoe (University of California, San Diego School of Medicine, USA) described two cellular phenotypes that distinguish bipolar lithium responders from nonresponders using samples in the Pharmacogenomics of Bipolar Disorder Study. As it is cost prohibitive to phenotype very large samples for drug response for GWAS, an efficient strategy may be to use cellular models to identify a set of candidate genes that are more likely to be involved in drug response, and then test those genes for association to response by targeted sequencing or genotyping. In their study, iPSCs derived from lithium responders, matched nonresponders, and controls were differentiated into PROX1-positive hippocampal dentate gyrus/glutamatergic neurons. Patch-clamp studies showed extended trains of action potentials in responder lines and similarly in nonresponder lines, but not in control lines. They also observed a corresponding alteration in calcium flux. The hyperexcitability by both electrophysiology and calcium flux measures was rescued by lithium, but only in responders. Lymphoblasts from lithium responders and nonresponders were treated with lithium and gene expression was compared within each patient. Results showed that nonresponders show a markedly reduced gene expression response to lithium compared with responders, which suggested that the clinical response to lithium is because of a much greater change in the physiological state in the cells. The most notable differentially expressed genes were $C R I P 2, A H N A K, A N T X R 2$, and MYADM. These current findings supported lithium-responsive BD to be a mechanistically distinct form of illness. A WGCNA is in progress.

\section{(Epi)genetics and (epi)genomics of psychological treatment response (reported by Marcos L. Santoro)}

Jonathan Coleman (King's College London, UK) began the session by presenting the first GWAS to examine the differential response to cognitive behavioral therapy (CBT) in a cohort of children with anxiety disorders. The GWAS was completed on 980 children after CBT, and although they did not find any SNP that reached GWAS significance, they raised some findings above suggestive significance $\left(P<5 \times 10^{-6}\right)$ that are near $( \pm 100 \mathrm{~kb})$ candidate genes such as VAV3. He discussed that these variants involved in response to CBT probably have small effect sizes, and thus this lack of significance is probably because of the sample size, which indeed is large for a study of response therapy, but still small for a psychiatric GWAS.

Dr Susanna Roberts (King's College London, UK) presented her work on gene expression in response to exposure-based CBT. They have analyzed the genomewide expression using Illumina Human HT12 V4 Expression BeadChip in 78 adults at three time points (pretreatment and post-treatment, and follow-up). Using Eigengene Networks, they generated nine modules in pretreatment expression data and associated them with clinical traits (sex, age, remission time point, and changes in severity). After comparing the expression characteristics of treatment responders and nonresponders, they found some enriched pathways such as neurotrophins and neurotrophic signaling, cytokines, and immune system; these pathways have been implicated in anxiety disorders. 
Dr Michael Meaney (McGill University, Canada) substituted for Rachel Yehuda (Mount Sinai School of Medicine, USA) and spoke about her recent findings in promoter methylation of glucocorticoid-related genes ( $N R 3 C 1$ and $F K B P 51)$ in response to posttraumatic stress disorder (PTSD) treatment. This was a preliminary test with 16 completers of prolonged exposure psychotherapy at three time points (pretreatment, post-treatment, and follow-up) to support a large trial (Yehuda et al., 2013). They found that methylation in the promoter of $N R 3 C 1$ exon $1_{\mathrm{f}}$ predicted treatment outcome (more methylated in responders' pretreatment compared with nonresponders). For FKBP51, they found a decrease in methylation of exon1 promoter associated with recovery.

Dr Elisabeth Binder (Max Planck Institute of Psychiatry, Germany) presented findings in blood gene expression to find predictors for major depression involved in the response to CBT, antidepressant drugs, or deep brain stimulation. They used the Illumina HT12 V4 array to verify the gene expression at different time points and found that from the top 72 transcripts of each treatment, more than a half of them had a binding site in the mycassociated zinc finger protein (MAZ) transcription factor. They also found that MAZ itself showed increased expression over the time of treatment. Thus, she showed that MAZ could be a biomarker of recovery, but not a biomarker for a specific treatment response.

Dr Thalia Eley (King's College London, UK) ended the session by discussing where we are and where we are going in the still growing field of therapy genetics. She highlighted the importance of prediction of treatment response and mentioned that $\mathrm{G} \times \mathrm{E}$ should be better explored and that we should clarify the exact mechanisms involved in these specific alterations.

\section{Oral sessions}

\section{The genomics of affective disorders and ADHD (reported by Maximilian Friedrich and Alex Shaw)}

Joanna Martin (MRC Centre for Neuropsychiatric Genetics and Genomics, Cardiff University) discussed to which extent common and rare genetic variants contribute toward an ADHD phenotype. Given the strong heritability (76\%) of the disease, she underlined that even though the burden of large and rare CNVs is significantly higher in children with ADHD, common genetic variants can be used to distinguish ADHD cases from controls. Thus, both types of variants may contribute toward the disorder. She concluded that rare CNVs are independently predictive of ADHD case status, which accords to the polygenic liability threshold model postulated for ADHD.

Christel M. Middeldorp (VU University Amsterdam, Biological Psychology) carried out a bivariate twin analysis to investigate whether ADHD PRSs enable prediction of the most common ADHD comorbidities such as aggressive behavior as well as anxiety and depression. Middeldorp pointed out that for the aggressive phenotype, a consistent prediction by the ADHD PRS could be made between 3 and 18 years of age, but not so for the anxious depression phenotype. The high monozygotic scores in the twin correlation study suggest that this is an effect of common genetic factors, whereas for anxious depression, a common environmental risk seems more likely. She put in perspective that 
these findings may be because of 'cross-assortative mating', a phenomenon explaining that patients with high ADHD scores preferentially mate, with more anxious individuals leading to an increased comorbidity of those conditions. Following her talk, the question arose as to why bivariate analyses were used instead of multivariate analyses. Middeldorp clarified that the latter approach can be used to identify associated genes for both phenotypes without allowing further differentiation, whereas the bivariate approach enables a more concise discrimination of genetic factors contributing toward both comorbidities.

Evie Stergiakouli (MRC Integrative Epidemiology Unit at the University of Bristol) investigated whether the same genetic variants contribute toward both behavioral traits in a control sample as well as to clinically relevant traits in an ADHD sample. Stergiakouli compared PRSs of ADHD symptoms in a general population sample and an ADHD sample and elucidated whether they could predict the diagnostic status and severity of the disorder. The main findings of this analysis were that PRSs are associated with the total number of ADHD symptoms and furthermore can distinguish between clinical samples and the general population. She concluded that the same common genetic variants for ADHD traits in the general population are implicated in clinical ADHD and that they predict both severity and ADHD diagnostic status. She also suggested that additive genetic influences are relevant for complex behavioral traits in both general population and clinical ADHD samples.

Dr Najaf Amin (Erasmus MC, the Netherlands) discussed her work aimed at identifying rare genetic variants that confer large effects on depression. From a large family of Dutch descent spanning 23 generations, 1686 individuals were exome sequenced and 1522 individuals were genotyped on an exome variation SNP chip. Several approaches were used to identify relevant variation including linkage, haplotype reconstruction, and association analyses, with three variants of interest identified. A missense variant was found on chr9p24 $\left(P=9 \times 10^{-4}\right.$, $\mathrm{MAF}=1 \%$, effect $=2.47$ ) linked with a high Hospital Anxiety and Depression Score. The authors also found a variant with a significant single-variant genome-wide association with Hospital Anxiety and Depression Score on chr11q13 $\left(P=9.2 \times 10^{-8} ; \mathrm{MAF}=1.7 \%\right.$, effect $=$ 3.36). The gene harboring this variant is expressed in specific brain regions, is functionally associated with neurotransmitters relevant to depression, and has been implicated in BD. A highly conserved missense variant on chromosome 18q21 was significantly associated ( $P=$ $1.3 \times 10^{-9}, \mathrm{MAF}=1 \%$, effect $=4.37$ ) with Centers for the Epidemiologic Studies of Depression score. The variants in 9p24 and 18q21 failed to replicate in the independent replication dataset, whereas the variant in the 11q13 region was not found in any replication dataset, which highlights one of the difficulties in validating genetic effects of putatively functional rare variation across studies. Future functional work will involve the creation of iPSCs and study of their metabolomic profiles.

Professor Dr Sven Cichon (University of Basel) presented results from a pathway-based analysis of a large BD GWAS dataset. The motivation for this and similar studies is that variants associated with psychiatric disease so far have small effect sizes, which are difficult to detect using GWAS with currently available sample sizes and that knowledge of each associated gene alone may not individually yield deep insights into disease biology. An interval-based enrichment (INRICH) pathway analysis was carried out on genes derived from a GWAS of 9747 patients with BD and 14278 controls, which represents the combined 
GWAS data from PGC1-BD and BD-cohorts from the MooDS consortium, and is the largest BD GWAS dataset to date (Mühleisen et al., 2014). Genes were tested for enrichment within 430 sets on the basis of biological pathways including 3881 genes (Reactome; http:// www.reactome.org). A subset of 10 intervals, corresponding to a single gene each, was enriched for the Reactome pathway 'neural cell adhesion molecule signaling for neurite outgrowth'. This subset was the only pathway that survived the full multiple testing procedure $(P=0.002)$. There are multiple lines of support for the involvement of the NCAM pathway including the presence of genes with significant association from BD GWAS (CACNA1C, NCAN, and CREB1; Mühleisen et al., 2014), candidate gene studies showing an association, and postmortem brain gene expression studies. Future work will involve evaluating different $P$-value thresholds for inclusion of GWAS SNPs in the analysis using alternative methods for enrichment analysis and using protein-protein interactions to define the target gene sets with potential enrichment.

Lower EA has been associated with a higher risk for depression (MDD) in multiple studies; however, the effects that contribute toward this association and the direction of effects within this association remain unclear. Major hypotheses are that low EA has a causal effect on risk for MDD, MDD has a causal effect on EA, or a third factor exerts pleiotropic effects on both MDD and EA. Dr Wouter J. Peyrot (VU University Medical Center \& GGZ inGeest, the Netherlands) discussed his study in which he aimed to evaluate the hypothesis of a pleiotropic genetic effect contributing toward the association between low EA and high risk of MDD. Using logistic regression, EA was associated with MDD in their dataset as expected, with an effect size of 0.85 per SD increase in years of education (95\% confidence interval 0.82-0.88). This association was also found using only MDD cases with an AAO of greater than 30 years. However, no consistent evidence was found for pleiotropic genetic effects. PRSs were predictive within either EA or MDD, but not when using one dataset as the discovery sample and the other as the target sample. Given the association between lateonset MDD and EA, the causality of MDD on EA seems unlikely. It is possible that there is a relatively low genetic correlation that the study had insufficient power to detect or that EA exerts an effect on risk for MDD. Future work in this area could examine environmental factors, such as socioeconomic status, that may account for pleiotropic effects.

\section{Epigenetic approaches (reported by Denise Haslinger and Luca Pagliaroli)}

Sarah Marzi (King's College London, UK) presented her findings on allele-specific methylation (ASM) in brain and blood samples. Although methylation is symmetric on both alleles across most of the human genome, there are several studies reporting that ASM is a common phenomenon (Schalkwyk et al., 2010). In their discovery sample, they tested postmortem brain and whole-blood samples of three control individuals. By measuring the change in the relative allele score, they found a cerebellum-specific ASM in rs959246.

About $4 \%$ of the informative SNPs showed allelic skewing with a change in relative allele score more than 0.1. They observed a strong and tissue-specific ASM in blood and cerebellum, whereas the cortex showed uniformly low ASM. In the replication sample ( $n=$ 38), they found differentially methylated regions in the vicinity of the top 100 tissue-specific ASM sites. In addition, they identified regions of hemimethylated probes near polymorphic ASM sites and showed that genotype-driven ASM can be tissue specific. 
Dr Chloe Chung Yi Wong (King's College London, UK) performed a comprehensive epigenome-wide scan for autism susceptibility loci across three brain regions (dorsolateral prefrontal cortex, primary auditory cortex, and cerebellum) in 218 postmortem brain samples of ASD patients. She observed significant ASD-associated DNA methylation differences in both novel genomic regions as well as in vicinity of several known ASD genes. Disease-associated epigenetic differences were found likely to be brain region specific. By carrying out a WGCNA, Dr Wong identified a number of significant ASDassociated differentially methylated modules that showed enrichment for neurodevelopmental pathways.

A recent study on gene-childhood trauma interactions showed that dexamethasone (Dex) treatment of human hippocampal progenitor cells (HPCs) induces GR activation, leading to a specific demethylation at intron 7 of FKBP5 (Klengel et al., 2013). Here, Dr Nadine Provençal (Max Planck Institute for Psychiatry, Munich, Germany) presented a genomewide analysis of the epigenetic effects of GR activation in HPCs after Dex treatment. This treatment had a broad impact on DNA methylation and hydroxymethylation and could induce specific DNA methylation and gene expression changes, some of which are long lasting. A comparison of their findings with an epigenomic analysis of probands with a history of child abuse (Dunlop et al., 2012) indicated an overlap of DNA methylation in Dex-treated HPCs and proband blood samples.

Dr Mill's (University of Exeter) research suggested a strong correlation between ANK1 hypermethylation and AD-associated neuropathology. In a comprehensive study of 120 individuals from the MRC London Brain Bank for Neurodegenerative Disease (KLC), the genomic DNA of four different brain regions has been investigated. In particular, he focused on the entorhinal cortex, PFC, superior temporal gyrus, and cerebellum. Three replication cohorts have been used from the Mount Sinai Alzheimer Disease and Schizophrenia Brain Bank ( $n=144)$, the Optima/Oxford Study $(n=61)$, and the Harvard/Religious Order Study $(n=708)$. The ANK1 correlation was confirmed from both the second and the third replication, which showed an AD-associated hypermethylation across an extended region of the brain. Meta-analyses across the three sample cohorts (London, Mount Sinai, Oxford) confirmed the neuropathology associated with cortex-specific hypermethylation for ANK1 CpG sites (cg11823178, cg05066953). The fourth study (Harvard) confirmed the previous findings once again. These data provide compelling evidence of an association between cortex-specific ANK1 hyper-methylation and AD-related neuropathology.

Dr Spiers (King's College London) analyzed a cohort of fetal brain tissues to investigate the level of DNA methylation during brain development. The analysis of 179 samples from the fetal cohort showed that $43.6 \%$ of sites showed hypermethylation, whereas $56.4 \%$ showed hypo-methylation. Another interesting result is that, although the majority of sites were located on the X-chromosome, 521 autosomal sites showed sex differences in DNA methylation. Weighted gene comethylation network analysis identified 22 modules of comethylated loci enriched for functional pathways associated with fetal brain development. In conclusion, this study provides increased knowledge of the molecular mechanisms that regulate dynamic gene expression across human brain development. Moreover, it identifies pathways contributing toward sexual differentiation of the brain. 
Starting from previous studies that showed a hypermethylation of $\mathrm{CpG} 3$ and $\mathrm{CpG} 4$ at the $S L C 6 A 4$ promoter in both lymphoblastoid cells and brain specimens from patients with $\mathrm{BD}$, Dr Ikegame (the University of Tokyo) investigated DNA methylation in blood samples of patients with BD. The ratio between 456 controls and 448 cases separated by sex showed a hypermethylation at CpG3 in male BD as reported previously by Philibert et al. (2008). The comparison of the DNA methylation ratio at $\mathrm{CpG} 3$ separately by genotype showed a hypermethylation in $\mathrm{L}$ variant carriers of male BD. Further analysis on the basis of $\mathrm{L}$ variant subtypes showed a specific SLC6A4 hypermethylation in male L variant (16-3) carriers with BD. Furthermore, a luciferase assay showed enhanced gene expression in the SLC6A4 promoter region.

\section{Novel 'Omics' insights into schizophrenia and bipolar disorder (reported by Siri Ranlund and Antonio Pardiñas)}

Robert Power (Institute of Psychiatry, King's College London, UK) reported findings from a PRS analysis investigating the link between psychiatric illnesses and creativity. PRS for SCZ and BD were calculated from the PGC results (Psychiatric GWAS Consortium Bipolar Disorder Working Group, 2011; Schizophrenia Psychiatric Genome-Wide Association Study (GWAS) Consortium, 2011). The target sample included 104220 Icelandic individuals (1312 individuals in creative industries; 521 schizophrenic patients, 680 bipolar patients, and 92647 population controls). The creative individuals included chess players, painters, writers, musicians, and dancers. Results showed that both SCZ and bipolar PRS significantly predicted creativity. Thus, individuals with high genetic risk for these disorders were more likely to be creative. Bipolar (but not SCZ) PRS also predicted whether an individual goes to university as well as higher grades. Hence, bipolar genetic risk seems to be associated with increased cognitive ability. Replication of these findings is currently underway. Power concluded that common variants for bipolar illness and SCZ may not be entirely deleterious and that bipolar PRS appears to be linked to higher cognitive functioning, whereas SCZ PRS is not.

Dr Manuel Mattheisen (Centre for Integrated Register-based Research, Aarhus University, Denmark), on behalf of Professor Esben Agerbo, reported results from a study investigating the link between family history and PRSs in the risk of developing SCZ. PRS for SCZ were calculated using results from the PGC mega-analysis (Schizophrenia Working Group of the Psychiatric Genomics Consortium, 2014). The target data set was a Danish population-based sample, including 866 schizophrenic patients and 871 controls. Family history was defined as those with a first-degree relative with a psychiatric illness. Results indicated that there is an interaction between family history and PRS. A family history of SCZ/psychosis had only a limited impact on the risk of developing SCZ among patients with low genetic liability, but the impact increased rapidly with increasing liability. Mediation analysis showed that up to $47.7 \%$ of the effect of family history was mediated through PRS. For the remaining52.3\%, different explanations are possible, including shared environmental risk factors and undetected genetic risk variants, and most likely a combination of the two. Mattheisen noted the limitations of the study including the small sample size and the fact that the PRS analysis does not take into account all genetic risk factors (i.e. rare variants). 
Dr Stephan Ripke (Massachusetts General Hospital, Boston, USA) reported an update of the recent SCZ PGC results (Schizophrenia Working Group of the Psychiatric Genomics Consortium, 2014), finding more than 100 significantly associated loci. Among the associated variants are calcium channel genes, which could provide possible novel drug targets, as well as glutamate-related genes and dopamine DRD2 receptor genes, both in line with hypotheses of SCZ. However, there are also associated regions with yet unknown biological functions and there are several methods available to investigate this. Tissues showing enrichment include the nervous system and also the immune system, again in line with hypotheses of SCZ pathogenesis. Enriched gene-set analysis can identify candidate genes for further investigation. Ripke concluded by arguing that the PGC has been successful with its core work in identifying new risk variants for SCZ. However, the secondary analyses, to investigate the biological mechanisms linked to the identified risk loci, require more work. This is currently underway by several collaborators.

As a representative of the Commonmind Consortium (http://www.commonmind.org), Professor Pamela Sklar (Icahn School of Medicine at Mount Sinai) summarized their preliminary first analysis of 700 postmortem brain samples from SCZ patients and healthy controls. These samples were genotyped on an OmniExpressExome platform, which, after quality control, delivered a median of 16423 genes valid for analysis in each sample. These data were supplemented by chromatin immunoprecipitation sequencing and RNA sequencing procedures, which provided expression data. Differential expression was found between cases and controls, with 199 downregulated genes and 267 over-regulated genes in cases, including members of the Ephrin and Semaphorin families, and the Slit-Robo pathway. A possible duplication of the KCTD13 gene was also found in cases, which has been previously linked to changes in cell proliferation and apoptosis (Golzio et al., 2012). Genome-wide patterns of expression showed depletion of expression quantitative trait loci (eQTLs) in intergenic regions, which might be related to SNP associations found in the most recent GWAS of SCZ (Schizophrenia Working Group of the Psychiatric Genomics Consortium, 2014). Finally, an analysis pipeline using Bayesian Causal Expression Networks was shown, which might potentially lead to increased detection of functional connections between causal genes. The full details of data generation and analysis of this project are available on Synapse (http://www.synapse.org).

Dr Douglas Ruderfer (Mount Sinai School of Medicine) reported the extended analysis of a Swedish cohort of 4978 SCZ cases and 6256 healthy controls that had been screened previously for psychiatric CNVs (Szatkiewicz et al., 2014). The new methodology included exome sequencing and indel calling by a hidden Markov model (Poultney et al., 2013) in an attempt to detect a possible burden of rare variation in particular genes. Although no novel genetic regions were found with a significant presence of CNVs, an overall burden of these was found in cases respective to controls. Also, no CNV enrichment was found on gene-set tests, but the analysis of trio families pointed to the presence of deletions in calcium channel genes, found in six cases and 0 controls. This dataset is currently being supplemented by the addition of roughly 5000 additional individuals.

More details from the same Swedish cohort were provided by Dr Giulio Genovese (Broad Institute of MIT and Harvard), who reported on the assessment of somatic mutations. 
Previous analyses had reported subtle differences in LoF mutations between cases and controls, with some of these being within brain expressed genes. As most of the LoFs were population singletons, they were considered to be de novos, but they could be as recent as to have occurred in embryonic or postembryonic phases. These somatic mutations are not heritable but, depending on the tissues in which they manifest, can have an impact on clinical phenotypes. By the analysis of allelic fractions (Carter et al., 2012), somatic mutations can be detected in sequence data from blood because of their amplification by clonal expansion in hematopoietic tissues. In the Swedish cohort dataset, 3111 somatic mutations were found, all of them singletons. These mutations constituted LoFs in several genes related to tumor suppression in leukemia (DNMT3A, TET2, and ASXL1) and were themselves associated with a 13 -fold risk of this disease. The raw number of somatic mutations was found to be increased in SCZ cases (odds ratio $=2.2$ ), which may be related to the increased prevalence of cigarette smoking in patients with SCZ.

\section{Genomic approaches in schizophrenia, substance abuse, and post-traumatic stress disorder (reported by Giorgia Quadri and Stefanie Malan-Müller)}

Dr Duncan (Broad Institute at MIT and Harvard) presented the first results of a GWAS on PTSD carried out by the PGC. PTSD is a common psychiatric disorder. Its phenotype depends on experiencing a traumatic event and its symptoms involve the re-experiencing of the traumatic event and having increased physiological arousal such as hypervigilance in response to the trauma. Although the biology of this condition is considered to be well understood, genetic variants contributing toward the heritability of PTSD are still largely unknown. To gain more insight into the genetic architecture of this disorder, the PGC formed a PTSD group to perform a GWAS. The interesting aspect of the datasets used for this GWAS on PTSD is the ancestry. A typical PGC dataset predominantly comprises European samples; the PTSD dataset instead included six African American datasets, six European American datasets, and one Hispanic origin dataset. The challenge of this was the absence of a method to assign ancestry in a way that is replicable and standardized - this is normally dealt with by carrying out a PCA with reference populations. Duncan and her group developed a methodology relying on SNP weighting to overcome this issue. The first stage of this analysis involved a meta-analysis across the African American dataset, one across the European American sample, and finally a meta-analysis across the three ancestry groups. In the meta-analysis carried out across the three ancestry groups, there were two loci that crossed the threshold of genome-wide significance; one of them appears to be a false positive, but the other locus did not. This SNP found in the intergenic region of chromosome 4 has a P-value of $3.76 \mathrm{E}-8$ and a MAF of $8 \%$.

The other interesting aspect of this study was the sign test between SCZ and these datasets; indeed, both in the African American and in the European American datasets, there were stretches of genome with a significant $P$-value. $3: 4$ showed significance for the European American; $1: 4$ showed significance in the African Americans, whereas in the Hispanic cohort, the sign test was not significant, likely because of the small sample. It is also important in terms of equity in science that diverse samples are included in the projects. 
Dr Duncan and her team hope to follow the trajectory of other similar studies to gain more insight into the genetic architecture of PTSD.

Dr Benros (Mental Health Centre, Copenhagen) carried out a study on the association between infections and SCZ and the influence of PRSs. Several studies have suggested an important role of infections and immune responses in the etiology of SCZ. However, the causal pathway underlying an increased risk of SCZ in individuals with infections is still unknown. SCZ presents itself with a series of immune alterations such as increased cytokines, increased autoantibodies, dysfunction of the blood-brain barrier, and low-grade nerve inflammation.

Benros and his group used the Danish registers (1981-2008), which, together with the psychiatric registers, allowed the identification of 39076 individuals with SCZ. The cases utilized for this study included individuals with a diagnosis of SCZ from 1994 to 2008 born from 1981; matched controls were identified in the registers. All individuals analyzed were adjusted by age, sex, and date of birth. Results showed that individuals with autoimmune diseases and three or more hospital contact infections had 3.5 times increased risk of developing SCZ. The same pattern was observed when excluding individuals who used substances of abuse and those with a psychiatric family history. This association could be because of the genetics of the shared risk factors but could also be explained by the possibility that individuals affected by SCZ may be more genetically vulnerable to infections causing the development of SCZ.

The polygenic scores were based on the PGC discovery data without the Danish population and the Danish replication sample and included 1697 schizophrenic patients and 1725 controls. The Danish replication study showed a significant association between the polygenic score and SCZ, and when used as a continuous variable, it increased the risk of SCZ by $47 \%$. It was shown that $40 \%$ of patients with SCZ had previous hospital contact with infections and that hospital contact with infections increased the risk of developing SCZ by 55\%. When dividing the PRSs into quartiles, it was shown that the upper quartile of the polygenic score was associated with SCZ by 2.29 times in individuals with no hospital contact with infection and by 3.36 times in individuals with previous hospital contact with infections. Instead, when the PRS was used as a continuous variable, it was shown that in individuals who had previous hospital infection, the risk for SCZ was increased by $44 \%$, whereas in individuals who did not have hospital infections, the risk decreased by $36 \%$. There was no interaction between the effects of the infections and the PRS and the risk of developing SCZ.

The study itself has several strengths such as having a prospective study design using population-based registers, and having data obtained by following a large population longitudinally in time with follow-up. The limitations instead include the sample inclusion being based on clinical diagnosis rather than criterion-based diagnosis, but previous studies have confirmed the validity of the information recorded in the registers. In conclusion, infections are associated with an increased risk of developing SCZ also after adjusting for the polygenic score, which did not influence the association between infections and SCZ or the risk of acquiring infections. 
Dr Paciga's presentation aimed to summarize some of the work of the Neuroscience Group from Pfizer carried out in the last year to identify new targets for $\mathrm{SCZ}$ and to explain how human genetics is applied to the discovery of new targets for drug development and the process established for triaging novel loci mechanisms for SCZ.

Human genetics can be applied to all stages of drug development, with a focus primarily on discovery genetics, and to clinical genetics; human genetics is applied to identify and also validate new drug targets and to help in the development of new mechanistic therapeutic hypotheses. The process developed by the company to go from risk loci to a new medical entity involves the definition of a phenotype and or endpoint that is strongly associated with a locus/loci. Then, the locus is mapped to a gene and insight has to be gained on how that gene can be causal or protective for the disease. The identified genes then have to be considered in the biology of the disease with a final need to identify a testable therapeutical hypothesis that can be tested.

The PGC has a very productive SCZ working group that has been in place for several years now; Dr Paciga and the Neuroscience Group from Pfizer joined this group in 2012 using data generated using a SCZ case-control dataset. As a result of the PGC release 2, 125 genomewide significant loci were identified. However, a list of loci does not equal a drug target; thus, a process was needed to obtain associated loci to test in a clinic.

A group that included geneticists, clinicians, and chemists was established to map those loci to specific genes. The process involved taking the 125 loci and using an LD gene mapping process with the assumption that each independent locus would be associated with one causal gene; the list was then narrowed down to 114 genes; and the resulting genes were then annotated to other available data including eQTL data for functional variants within those genes and previous literature knowledge was used to understand what could be associated with those genes. With manual curation, the list went down to 14 lead genes; subsequently, through a manual process using other available data and literature in consideration of animal models and knockouts, a list of around five priority genes was produced for the follow-up. Finally, with target reviews of animal models, the aim is to get to 1-2 target genes.

This method was first used for cardiovascular disorders and performed well for SCZ too. The loci identified here as enriched were for biological functions related to SCZ; in addition, the genes identified were enriched in relevant tissues with a two-fold enrichment of these genes for high brain expression.

Dr Younes Moukrab (Eli Lilly, UK) developed a pipeline to aid in the functional annotation of significant variants identified during GWASs for further biological analysis. The pipeline uses Variant Effect Predictor (http://www.ensembl.org) to annotate variants and their associated genes together with publicly available data on various functions and characteristics of the gene. The pipeline was applied to summary data of the Schizophrenia Working Group of the Psychiatric Genomics Consortium (2014) study that identified 108 distinct loci associated with SCZ, of which 84 have not been implicated previously in SCZ. Variant Effect Predictor analysis identified 268 genes for which variants were located 
outside gene boundaries; for 36 genes, the variants were located inside a gene and a number of genes were affected by missense variants, including SLC39A8, APOPT1, ITIH3, and $F E S$. These data can help to build specific hypotheses on changes in gene function and subsequent associations with SCZ etiology.

Using one of the items on the Fagerstrom test for nicotine dependence (FTND), the time to smoke the first cigarette in the morning (TFC), Dr Xiangning Chen (Virginia Commonwealth University, USA) and colleagues at the FTND consortium identified risk genes for nicotine dependence. The consortium included 15 independent datasets and over 19000 European individuals. Genotype imputation and association analyses were carried out at each site independently, and meta-analyses were used to combine data from individual datasets. Results indicated that the a 5 nicotinic cholinergic receptor (neuronal) (CHRNA5$A 3-B 4)$ gene cluster was associated with FTND scores as well as the TFC phenotype. TFC analyses identified three new loci, sorbin and SH3 domain-containing 2 gene (SORBS2), and two long noncoding RNAs, BG182718 and AA333164; the latter was replicated in independent samples. Some of the other top candidate genes (supported by both FTND and TFC analyses) have previously been found to be associated with smoking behaviors [cholinergic receptor, nicotinic, $\beta 3$ (neuronal) (CHRNB3)], cancers [cadherin 12, type $2(\mathrm{~N}$ cadherin 2) (CDH12), heparan sulfate (glucosamine) 3-O-sulfotransferase 4 (HS3ST4), and major facilitator superfamily domain containing 2A (MFSD2A)], obesity [kinesin family member 2B (KIF2B) and $C D H 12$ ], and psychiatric disorders [CDH12 and zinc finger protein $804 \mathrm{~A}($ ZNF804A)].

Professor Eske Derks (Academic Medical Center, Amsterdam) and colleagues carried out a meta-analysis on one of the largest GWAS on cannabis use/initiation. It included results from 11 discovery samples from Europe, the USA, and Australia and two replication samples in cohorts from the UK and the Netherlands. Their fixed-effects meta-analysis showed no genome-wide significant associations; however, there was enrichment of nominally significant findings. The most statistically significant marker was rs2099149, an intergenic polymorphism located close to the transmembrane and tetratricopeptide repeat containing 1 gene (TMTC1) and the importin 8 (IPO8) gene. These findings were, however, not replicated in the replication sample. Gene-based tests identified two significantly associated genes in the discovery sample, namely, interleukin 17B (IL17B) and prenylcysteine oxidase 1 like (PCYOX1L). IL17B plays a role in immunity and inflammation; again, these results could not be replicated in the replication sample. The initiation of cannabis use may be because of various factors, most of which are largely unknown. Future studies will focus on alternative phenotypes such as age at initiation, frequency of use, and cannabis use disorders.

\section{Novel approaches and tools for bench and bedside (reported by Luca Pagliaroli)}

Dr Steven McCarroll (Stanley Center of Psychiatric research, Broad Institute of MIT and Harvard) held a conference on DropSeq. Riding the motto 'less smoothie and more fruit salad', he developed a new approach that uses beads to 'isolate cells in millions of tiny droplets'. He developed a cell 'barcode' by a progressive split-pool synthesis on beads that, after 12 rounds, allows the generation of 16 million beads with different barcode sequences. 
In the DropSeq, the cells are lysed in droplets and the mRNAs are hybridized to the beads that are subsequently collected and amplified in a single reaction. After 100 experiments, it has been possible to distinguish 100000 transcripts per cells.

So far, it is possible to originate 10000 single cell libraries per day at $0.06 € /$ cell. The current challenge is focused on the understanding of (i) brain tissues and their development, (ii) pathological states (e.g. SCZ), and (iii) the relationship between genetic results and specific cell populations.

Dr Niels Tommerup (Wilhelm Johannsen Center for Functional Genome Research, University of Copenhagen) analyzed data from the Danish prenatal cohort seeking unselected carriers of de-novo balanced chromosomal rearrangements. It was possible to identify eight carriers among the neurodevelopmental/neuropsychiatric disorders that are the main causes of deletions, inversions, or translocations. The first unbiased mutagenesis screen (10 000 breakpoint; 150 laboratory; 50 countries) provided evidence that the screening becomes more cost-efficient with a database search for known mutations. Indeed, this study identified 2700 protein-coding genes; 1500 long noncoding genes; less than 200 regulatory domains specifying the body plan; and more than $20 \%$ associated with NDD.

Dr Stephan Ripke (Massachusetts General Hospital) presented the PsychChip. The aim of this project is the genotyping of $100000+$ psychiatric disease samples. Its goals are to find new associated loci, to determine the rate of rare mutations, and estimate the role of CNVs. The PsychChip is designed with 50000 SNPs (PsychSNP), a 256000 SNP GWAS Backbone, and 240000 rare and low-frequency exome variants (exome chip). Each sample costs $\$ 83$. The custom content accounts for (i) all independent signals with P-value less than 0.01 in any study (25 702/38 178) and (ii) the fine mapping of loci with P-value less than $1 \times$ $10^{-5}$ (4498/7242). Its efficiency derives from the choice of SNPs that are already known in psychiatric diseases with SCZ as the front runner (23\%) for GWAS-significant findings.

There are three steps to data generation: (i) genotype calling using Birdseed and GenCall; (ii) merging the data, followed by further genotype calling using zCall (rare variant); and (iii) final elaboration (QC). This method proved to perform well even at a lower price compared with other chips available on the market. So far, 40268 samples have been genotyped and the data are available at http://www.med.unc.edu/pgc/psychip.

Dr Rebecca Knickmeyer (UNC Chapel Hill) focused her attention on GWAS studies. One of the goals is to investigate whether common SNPs are associated with global brain volume at 2 weeks of age. 'By the time the symptoms arise everything has already happened', and for this reason, it is important to study infants. Indeed, the prenatal period is the 'main window' of brain development and strong evidence suggests that multiple diseases have their origins in early life (in the first year of age, the brain volume has already reached $82 \%$ of its final dimensions).

In a comprehensive study of tissue volumes of 988 neonates, different loci have been identified as able to increase the mental disability rate, including rs8030297 on chr15q13.3, RBFOX2 (cerebrospinal fluid), DPYSL3-ZNF385D (cortical white matter), and 13q14.2q14.1 (cortical gray matter). Future studies should investigate (i) the surface area and 
cortical thickness, (ii) functional connectivity, (iii) DTI phenotype, (iv) $\mathrm{G} \times \mathrm{E}$ interactions, and (v) exome sequencing in extreme phenotype.

Dr Emma Sprooten (School of Medicine, Yale University) focused her attention on the study of valid endophenotype to discover, localize, and validate risk variants. A source-based morphometry has been applied to investigate the endophenotype of gray matter in several studies. Sprooten showed evidence for linkage of 12q24 to gray matter in the insula and $\mathrm{mPFC}$, variation of gray matter in the insula and $\mathrm{mPFC}$ between individuals, and suggested that reductions of gray matter may be associated with SCZ. Therefore, insula and mPFC morphology may be a neuroanatomical correlate to SCZ that is coinherited with SCZ susceptibility variants at $12 \mathrm{q} 24$.

Dr Bryan Dechairo (AssureRx Health) spoke on the use of combinatorial pharmacogenomics to avoid the worsening of patient conditions under medication treatment. He presented GeneSight, a genetic test that can analyze genes to help healthcare providers in selecting the medications that are more likely to work for patients. He compared this approach with three clinical studies: PineRest, Ham Clinic, and LaCrosse. In his opinion, medications can be divided into three categories: green (right), red (wrong), and yellow (uncertain). In the PineRest RCT study, almost $30 \%$ of patients in a typical psychiatric practice are taking red category medications. Treatment guided by GeneSight resulted in up to a four-fold greater improvement in symptoms. The use of 'red bin medication' results in an increase of $\$ 5188$ in healthcare utilization costs annually, whereas medication based on GeneSight results in a saving of \$1035.60/patient.

Dr Franke (Department of Human Genetics and Psychiatry, Donder's Institute of Brain, Cognition and Behaviour, Radboud University Medical Center) presented an overview of the Aggressotype project. As part of the EU FP7 program, the international consortium Aggressotype was founded in 2013 and focused on aggression and impulsive behavior in ADHD and conduct disorders. This project involves preclinical research at different levels: from genetics, to cells, to brain functions, and eventually to behavior. The following areas are under investigation: epigenetic studies of animal models to find biomarkers; imaging studies of animal models; alternative and classical animal model approaches suitable for behavioral studies and high-throughput screening; and NeuroIMAGE projects to study human behavior.

\section{Functional genomics and endophenotypes (reported by Jose Estrada and Stefanie Malan- Müller)}

James Crowley (University of North Carolina at Chapel Hill, USA) is studying how the antipsychotic drug olanzapine induces changes in the gut microbiome to induce metabolic side effects. In this study, a mouse model was utilized in which mice on a high-fat diet were administered either oral doses of olanzapine or placebo control. Mice in the olanzapine cohort showed a $20 \%$ increase in weight gain compared with non-olanzapine mice. The weight-gain effect is lost in olanzapine-treated mice that are bacteria free and raised in an antiseptic environment. Weight-gain effects in olanzapine-treated aseptic mice are regained if the mouse gut is allowed to be recolonized. Fecal pellets from olanzapine-treated and placebo control mice were genetically analyzed and decreased growth was identified in three 
species of gut microbiota: Bacteroidia, Erysipelotrichi, and Gammaproteobacteria. Analysis of in-vitro Escherichia coli growth when treated with olanzapine shows that the drug has antimicrobial properties.

Xueying Jiang (National Institute of Mental Health, USA) presented work that examines the effects of valproic acid (VPA) on Trank1 expression, a gene implicated by GWAS studies in bipolar individuals. Jiang analyzed four iPSC and seven NPC lines that were treated with placebo or VPA for $72 \mathrm{~h}$. VPA treatment on all cell lines increases Trank1 expression, whereas the risk allele rs9834970 of Trank1 is associated with lower expression. Lithium treatment did not increase Trank1 expression. The negative correlation between Trank1 and HDAC1 provides early evidence that Trank1 expression may be regulated by an epigenetic mechanism.

Stefanie Malan-Müller (Stellenbosch University, South Africa) studied the molecular mechanisms of D-cycloserine (DCS) in the extinction of fear in rodent models and relief of PTSD symptoms in humans. Left dorsal hippocampi tissue was taken from rodents that were subjected to a fear assay with and without DCS or no fear exposure with and without DCS, and analyzed for RNA and microRNA expression using next-generation sequencing technologies. Bioinformatics were utilized to identify microRNA-mRNA interactions specific to the fear condition-DCS treated rodent. One hundred and twenty-one genes and 32 microRNAs were predicted to be biologically significant for PTSD. Follow-up analysis on the candidate microRNA rno-mi31a-5p is being completed to identify whether it is capable of regulating a subset of the candidate genes.

Annika Forsingdal (Lundbeck A/S and University of Copenhagen, Denmark) investigated behavioral and transcriptomic alterations in homozygous $15 \mathrm{q} 13.3$ knockout $\left(15 \mathrm{q} 13.3^{-/-}\right)$ mice. The $15 \mathrm{q} 13.3$ hemizygous deletion $\left(15 \mathrm{q} 13.3^{+-}\right)$has been shown to strongly increase the risk of SCZ and other psychiatric diseases (Malhotra and Sebat, 2012) and animal models of this hemizygous microdeletion syndrome helped to identify disease-related phenotypes; the phenotypes were, however, subtle (Fejgin et al., 2013). The current study utilized homozygous 15q13 knockout mice to create stronger phenotypes to aid in the mechanistic understanding of the effects of this CNV. The knockout mice were characterized using basic physiological, behavioral, and disease-related behavioral tests. They also performed RNA sequencing of brain and body samples of knockout and wild-type mice. Basic and behavioral characterization showed that the phenotypes of the 15q13.3 knockout animals were similar to those observed in human homozygous carriers. The phenotypes of $15 \mathrm{q} 13.3^{-/-}$were more pronounced compared with that of $15 \mathrm{q} 13.3^{+/-}$. They also observed considerable transcriptional regulation. Suggestions were also made to focus on certain brain regions and not the whole brain for RNAseq.

Professor Abraham Palmer (University of Chicago, USA) used GWAS data from 398 healthy, nondrug abusing volunteers to investigate subjective responses to amphetamine (Hart et al., 2012). This response is heritable and may serve as an endophenotype for various psychiatric disorders. They used a double-blind, placebo-controlled, within-participant design and performed GWAS using genotypes from Affy 6.0 imputed to 1000 Genomes. Several putative associations were identified; however, there was no convergence on a SNP 
or LD block and none of these SNPs were coding variants or known eQTLs. An SNP in an intron of cadherin 13 (CDH13), rs3784943, was found to be associated with the positive subjective effects of amphetamine; this gene has been associated previously with various psychiatric traits, including methamphetamine (Uhl et al., 2008) and cocaine dependence (Gelernter et al., 2014). By comparing their data with the PGC1 SCZ dataset, they discovered that SNPs associated with the euphoric response to D-amphetamine are enriched among SNPs associated with SCZ (enrichment driven by alleles associated with increased euphoria and decreased SCZ risk) as well as SNPs associated with ADHD.

Lynsey Hall (University of Edinburgh, UK) investigated whether a quantitative endophenotype for MDD could be generated using cognitive, mood, and personality traits that are genetically correlated with depression. Data from a large family and populationbased study (21 340 individuals), Generation Scotland (GS:SFHS), were used. Three traits with the highest genetic correlation were used to create a composite trait, including the GHQ, Mood Disorder Questionnaire, and Eysenck Personality Questionnaire for Neuroticism. These were subjected to PCA. No genome-wide significant SNPs were identified; the top GWAS hit for the composite trait was in the $3^{\prime}$ intron of sushi domaincontaining protein 4 gene (SUSD4), rs4661250. SUSD4 is highly expressed in the white matter of oligodendrocytes and in MDD white matter integrity is reduced, therefore suggesting a possible role for SUSD4 in the etiology of depression. They also used summary data from the PGC-MDD GWAS and generated PRS profiles for MDD in GS:SFHS. The association between these scores and MDD and the newly derived composite trait were tested. The polygenic score had a stronger association with and explained more of the variance of the composite trait compared with depression or recurrent depression. This suggests that the trait captures a greater proportion of the heritable component of depression. However, this study cannot detect causal variants for depression as it is still under-powered. They hope to have a larger GWAS sample once the rest of the GS:SFHS cohort is included.

\section{Schizophrenia: genomics and more (reported by Giorgia Quadri, Denise Haslinger, Erik Loken, and Shing-Wan Choi)}

Dr Andrew Pocklington (Cardiff University) tested the impact of CNVs in SCZ. Individuals with SCZ have been found to have an increased burden of large and rare CNVs more than $100 \mathrm{~kb}$, frequency less than $1 \%$, compared with matched controls in several studies. So far, a few individual loci were strongly implicated more than $500 \mathrm{~kb}$, odds ratio (OR) 2-60, in $2.5 \%$ of patients and $0.9 \%$ in controls; most of these span multiple genes (NRXN1). Results from set-based analysis on case-control datasets showed enrichment for synaptic and developmental genes for SCZ CNVs. De-novo SCZ CNVs were instead enriched in postsynaptic $N$-methyl-D-aspartate receptor (NMDAR), which is central to the synaptic plasticity and ARC (neuronal activity-regulated cytoskeleton associated) protein complexes (Kirov et al., 2012); this was replicated in other CNV studies.

Pocklington studied CNVs in SCZ in a wider sample and collected European subsets to obtain 11917 cases and 16416 controls; the focus was on large (>100 kb) CNVs. The aim was then to look at previously implicated genes, most importantly, genes related to brain function and CNS functional development; 136 gene sets covering various aspects of 
behavior, memory, social interaction, brain region sizes, white matter sizes, cell morphology neurons, and glia were curated. Most of these were taken from the Mouse Genome Informatics phenotype database with a focus on single genes, functional genetic studies in mice, and neuronal annotation from proteomics studies. Finally, to test for any additional enrichment for these gene sets including large and rare CNVs, a logistic regression model with covariates was used to correct for CNV size, number of genes hit, and the inclusion of multiple studies and genotyping chips. For the three previously implicated sets, there was strong evidence for association with the NMDAR receptor complex. $P$-values were corrected for the three tests for deletions and duplications. The NMDAR signals mostly came from duplications (NMDAR duplication $P=7.53 \times 10^{-9}$ ); there was also a deletion signal in the neuronal activity-regulated cytoskeleton-associated protein complexes (ARC deletion $P=$ 0.001). Compared with permuted data, there was an excess of associated CNS gene sets that was also observed across several significance thresholds. Results included gene sets related to learning and memory, synaptic physiology, and postsynaptic protein complexes. Interestingly, there was a strong evidence of enrichment in the NMDAR complexes and in the PSD-95 (important scaffolding component of postsynaptic scaffolding molecule) complexes and $\mathrm{GABA}_{\mathrm{A}}$. Duplications and deletions showed the same results of the combined analysis.

Dr Brady Maher (Lieber Insititute of Brain Development/Johns Hopkins Medical School) aimed to study the function of genes during cortical development.

TCF4 is a basic helix-loop-helix transcription factor, which can regulate transcription. It is known to bind to E-boxes to either suppress or activate transcription. The main goal of this study was to understand which genes TCF4 regulates. The TCF4 region reached genomewide significance in several studies and some SNPs in this region have been associated with endophenotypes for SCZ including IQ. The Lieber Institute has been developing a dataset for RNA sequencing obtained from the dorsal lateral PFC of postmortem brains of 100 schizophrenic patients and 100 controls; this was used to understand the molecular mechanisms increasing the risk associated with TCF4 variants. The function of TCF4 was altered by in-utero electroporation to study its expression in developing neocortex in rats. The focus of the presentation was to describe the process of knocking down TCF4 expression using shRNA constructs. Two shRNA constructs were used: one that targeted the $5^{\prime}$

UTR and one that targeted an exon; this resulted in a $40 \%$ knocked down protein in neuroblastoma cells and in the rat neuroprogenitor cells. The basic protocol involved the development of DNA plasmids to knock down or overexpress a gene; these constructs were introduced through in-utero electroporation. This technique allows injecting DNA into ventricles of embryos; the DNA is then electroporated into the progenitor cells, which give rise to a pure population to layer two-thirds pyramidal cells. Next, functional analysis was carried out in acute brain slices using whole-cell electrophysiology and confocal imaging.

Here, it was shown that embryonic knockdown of TCF4 in two-thirds layer pyramidal cells caused a decrease in the intrinsic excitability and the ectopic appearance of spike-frequency adaptation. Both these phenotypes were associated with an increased afterhyperpolarization 
amplitude and were rescued by manipulating intracellular and extracellular levels of calcium. To rescue this phenotype, ribosome affinity purification was adapted to in-utero electroporation (iTRAP technique); this technique enables the purification of pure two-thirds pyramidal cells. Only two genes were found to be significantly upregulated: KCNQ1 (potassium voltage-gated channel) and SCN10A (sodium voltage-gated channel). In conclusion, it was shown that TCF4 regulates the intrinsic excitability of layer two-thirds neurons in a cell autonomous manner through regulation of the expression of specific ion channels (KCNQ1, SCN10A). Also, iTRAP has been shown to be a novel combination of techniques that allows for molecular profiling of the translator in a homogeneous population of transfected neurons. It was also shown that TCF4's regulation of transcription is context dependent; it is therefore likely that other neuronal cell types may have very different excitability phenotypes or none at all. This will be tested in TCF4 heterozygous mice.

Professor Peter Visscher (the University of Queensland/Queensland Brain Institute) investigated the role of paternal age and de-novo mutations in the risk of developing psychiatric illnesses. It is well established that advanced paternal age is a risk factor for psychiatric illness, with a relative risk of 1.2 for children of fathers 10 years older than the average. In addition, the rate of de-novo mutations, which are risk factors for psychiatric diseases, is higher in older men with approximately two extra mutations per year of advanced paternal age (Kong et al., 2012).

To address the question of whether older fathers have more mutations and thus children at a higher risk of developing psychiatric illness, Visscher created population genetic models and contrasted the model outcomes with empirical observations present in the literature.

The most applicable model is one where paternal age and genetic liability are correlated and there is assortative mating. In this scenario, the risk of psychiatric disease of children of older fathers is because of inherited rather than de-novo variants and a polygenic threshold model was assumed with a correlation between paternal age and his liability and between the liabilities of the spouses. This model very easily recreates the empirical observations found in real data. There is indeed a correlation of $5-10 \%$ between paternal age at first child and disease liability that generates a lambda for offspring of 1.2. Therefore, de-novo mutations on their own do not add up in explaining the empirical observations and the risk of paternal age is not predominantly from de-novo mutations (with or without selfish selection), but the correlation of paternal age and liability of the disease with or without assortative mating can explain the risk of children of older fathers of developing a psychiatric illness.

Thus, shared genetic factors between children and older fathers are a plausible explanation for the association between advanced paternal age and risk of psychiatric illness, but denovo mutations and additional nongenetic factors may also contribute. This modeling leads to some testable hypotheses such as testing the correlation between paternal age at first child and liability for psychiatric illness in the empirical data. Second, there could be a better integration of epidemiology and genetics in large samples such as deCODE to partition the risk to children because of paternal age, paternal age-related denovo mutations, and segregating genetic risk factors in large prospective population studies. 
Dr Joseph McClay (Virginia Commonwealth University, USA) presented his work on the SCZ-associated gene TCF4. Using chromatin immunoprecipitation sequencing, he aimed to characterize the TCF4 regulatory network in the neuronal cell line SH-SY5Y. Analysis of mock immunoprecipitation and input control comparisons implicated genes and pathways involved in neuronal functions such as neurite growth. McClay further reported that genes in the TCF4 regulatory network are more likely to be differentially expressed in brain tissue from SCZ or BD patients than in controls. Permutation testing of TCF4 binding sites within 108 genome-wide significant loci for SCZ reported in a study of the PGC (Schizophrenia Working Group of the Psychiatric Genomics Consortium, 2014) showed no significant enrichment. However, he could identify an overlap with SCZ-implicated genes that may suggest novel directed experiments.

Dr Simone Berkel (Institute of Human Genetics, Heidelberg, Germany) presented her work on the post-synaptic scaffolding protein $S H A N K 3$, which has been implicated previously in SCZ. To further elucidate the role of this gene in SCZ etiology, they sequenced the exons and flanking intronic regions of $S H A N K 3$ in 500 affected individuals and compared their results with ancestrally matched controls. Eight out of $500 \mathrm{SCZ}$ individuals carry rare SHANK3 missense variants that were not found in controls. To analyze the burden of rare deleterious $S H A N K 3$ variants, they combined their findings with previous studies on mutations of SHANK genes in ASD (Leblond et al., 2014) and SCZ (Gauthier et al., 2010), and reported a higher frequency of rare deleterious SHANK3 variants in ASD than in SCZ. Association analysis showed five SHANK3 variants with study-wide significance $(P<1 \mathrm{E}$ $-3)$ and one variant with genome-wide significance $(P<5 \mathrm{E}-8)$ for SCZ. Furthermore, they identified a $\mathrm{G}<\mathrm{V}$ (glycine to valine) variant of $S H A N K 3$ in SCZ and ASD probands, but not in controls.

Dr Jonathan Mill (University of Exeter, London, UK) discussed their systematic study of SCZ-associated methylomic variation in the adult brain and its relationship with changes in DNA methylation during human fetal brain development (Pidsley et al., 2014). They compared methylation of PFC and cerebellum samples in postmortem brains of SCZ patients with samples of controls and identified differentially methylated positions. On carrying out a WGCNA, they identified modules of comethylated loci associated with SCZ that are highly significantly enriched for genes involved in neurodevelopmental processes. Furthermore, they could show that SCZ-associated differentially methylated positions are significantly enriched for loci at which DNA methylation is dynamically altered during human fetal brain development.

Dr Daniel Howrigan (Massachusetts General Hospital) presented work on de-novo variation in 1109 Taiwanese trios with sporadic SCZ in the proband. He used exome sequencing and combined data with sequences from $2084 \mathrm{SCZ}$ trios and 698 control trios previously published in the literature. He found an enrichment of Lof $\left(P=6 \times 10^{-3}\right)$ and synonymous $\left(P=4 \times 10^{-6}\right)$ de-novo variants in cases. No single gene surpasses exome-wide correction. In a gene-set analysis of previously implicated gene sets (Fromer et al., 2014), the ARC complex genes had an enrichment of Lof mutations in the combined analysis $\left(P=8 \times 10^{-3}\right)$. Evolutionarily constrained regions (Petrovski et al., 2013) were enriched for Lof and 
missense mutations. The FMRP targets gene set was also enriched for Lof and missense mutations.

Dr James Walters (Cardiff University) presented research on the genomics of treatmentresistant SCZ. One-third of SCZ patients are treatment nonresponsive. He wanted to test whether these patients had an excess of polygenic burden, had a specific signal, or had increased CNV burden. He examined the polygenic burden in 5600 treatment-resistant SCZ cases and 6300 controls and no difference was found compared with generic SCZ cases from the PGC study. The treatment resistant cases on clozapine did have six new GWAS signals not in the original PGC analysis, which may be specific to treatment-resistant SCZ. DRD2 was more significant with the treatment-resistant cases in the analysis. No differences were found in CNV rates between treatmentresistant clozapine cases and generic SCZ cases. In summary, Walters found no polygenic signal difference between treatment-resistant SCZ cases and generic cases, but there may be specific loci for treatment resistance in SCZ. Walters was asked whether the DRD2 resulting from the PGC study (Schizophrenia Working Group of the Psychiatric Genomics Consortium, 2014) was entirely because of treatment-resistant cases within the case pool. Walters considered this a possibility as the signal was much weaker on removing samples taking clozapine from the analysis.

Dr Vanessa Gonçalves (Centre for Addiction and Mental Health, University of Toronto) presented research on the mitochondrial genome's association with the risk of SCZ. There are over 1000 nuclear-encoded mitochondrial genes and 37 mtDNA-encoded genes. Gonçalves examined SNPs in these genes in 9394 SCZ cases and 12462 controls of European ancestry from the PGC (Schizophrenia Working Group of the Psychiatric Genomics Consortium, 2014; Ripke et al., 2011). Using a stratified false discovery method, all original PGC associations were significant; in addition, associations with C6orf136, VARS2, and FTSJ2 replicated in an independent sample of 11 244. In addition, an oxidative phosphorylation gene-set analysis showed inflation in the P-values, indicating a role for variation with small effect sizes in the pathway impacting SCZ.

Dr Semanti Mukherjee (the Feinstein Institute for Medical Research, USA) presented the work of the Major Histocompatibility Complex-Psychiatric Genomics Consortium (MHCPGC2) working group. The working group attempted to use a subset of 38 cohorts of European ancestry (29 148 cases, 35483 controls) to pinpoint the MHC signal in SCZ. They followed the PGC2 framework for the analysis of the genotype data and carried out a stepwise conditional analysis, followed by meta-analysis using METAL. Imputation was performed using IMPUTE2 and SNP2HLA methods. By conditioning on the most significant SNP identified in PGC2 (rs115329265), two additional SNPs rs9461856 ( $P=$ $1.8 \mathrm{E}-8)$ and rs $115332348(P=4.2 \mathrm{E}-8)$ reached genome-wide significance. rs9461856 falls within the region of the $S Y N G A P 1$ gene, which associates with NMDA receptors at synapses, whereas rs115332348 falls within the HLA class II locus.

Jack Euesden (King's College London, UK) presented his work on the study of the relationship between SCZ and RA. The study included 1600 controls from the RADIANT study, 2000 RA samples from the WTCCC dataset, and 113075 controls and 36989 cases from the PGC2 SCZ study. Using PRSice (polygenic risk score software; http:// 
www.PRSice.info); it was found that there was no association between the polygenic score in SCZ and RA status.

Dr Karolina Aberg (Center for Biomarker research and Personalized Medicine, Virginia Commonwealth University, USA) presented her work on the study of an association between CpGs affected by common SNPs and SCZ disease status. Blood samples from 697 SCZ cases and 711 controls were used as the discovery data set, whereas the replication data set included a total of 75 postmortem brain samples (BA10, 26 SCZ cases, 22 bipolar cases, 27 controls). Using MBD-Seq, a total of seven differently methylated SNP-CpGs reached genome-wide significance in blood and one of the sites was also replicated in the brain sample. This site corresponded to the IL1RAP gene and the signal could be replicated in an independent set of blood samples using targeted pyrosequencing and SNP genotyping. By combining the discovery blood samples and brain samples, a total of four differently methylated SNP-CpGs were found to reach genome-wide significance; the IL1RAP site was among the significant sites. Three significant sites were validated in independent samples, whereas the last sites were not replicated because of technical difficulties in designing the primer for that particular region.

\section{Affective disorders: genomics and more (reported by Jie Song and Alex Shaw)}

Jie Song (Karolinska Institutet, Sweden) reported a family-based study using the Swedish population to investigate the familial risks for $\mathrm{BD}$ and its relation to other psychiatric disorders (Song et al., 2015). By linking several Swedish national registers, a total of about $50000 \mathrm{BD}$ patients were identified among eight million off-spring from four million nuclear families. Compared with matched-population controls, the familial risks for relatives of BD probands were 5.8-7.9 in first-degree relatives and decreased with genetic distance. There are also strong co-occurrence risks for other psychiatric disorders in individuals with BD and a relatively high risk in full siblings of BD probands. Heritability for BD was estimated at 58\% using a sibling model. The correlations between BD and other psychiatric disorders were considerable (0.37-0.62) and primarily because of genetic effects. The results are consistent with recently published molecular genetic studies (Cross-Disorder Group of the Psychiatric Genomics Consortium et al., 2013a, 2013b), suggesting the pleiotropic effects of genes across different psychiatric disorders.

Dr Eli Stahl (Icahn School of Medicine at Mount Sinai) reported their extended results from a meta-analysis of PGC2 BD GWAS including 10000 new case samples and 17000 new controls. Initial analyses yielded thirteen genome-wide significant loci, of which nine are new, for BD. Some of these new bipolar risk loci had evidence of association with other psychiatric phenotypes and were located in or near biological candidate genes (e.g. MPP6 in PGC2 SCZ, SSBP2 in PGC AN). The pathway analyses of the primary GWAS results again supported a strong overlap between BD and SCZ and roles for both calcium and potassium ion transport. Dr Stahl mentioned that the bivariate analysis of a subset of the new data shows that SNP heritability of BD1 is greater than that of BD2 and greater than BD1-BD2 coheritability, consistent with an incomplete genetic correlation ( 84\%) between BD1 and BD2 diagnoses. PRSs on the basis of other psychiatric disease GWAS differentiate between BD subphenotypes; for example, SCZ polygenic scores are higher in $\mathrm{BD} 1$ than $\mathrm{BD} 2$. 
Niamh Mullins (King's College London, UK) presented their investigation for interactions between polygenic scores and environmental adversity (i.e. childhood trauma and SLEs) in the etiology of MDD. They used the discovery results from a mega-analysis on MDD by the PGC to construct MDD polygenic scores for the individuals in the RADIANT UK validation dataset. They found that polygenic scores for depression showed significant predictive ability for depression in the RADIANT UK sample, whereas polygenic score-by-SLE interaction showed no predictive ability for case/control status. However, they found a significant interaction between polygenic score and childhood trauma. Interestingly, the regression coefficient showed a negative association. One possible explanation is that the environmental factor childhood trauma could be more important in the development of MDD for those individuals with a low genetic risk compared with those with a high genetic risk.

Dr Tim Bigdeli (Virginia Commonwealth University, USA) described his study, which examined the extent of the shared genetic basis for depression (MDD) across ancestrally divergent populations (European and Han Chinese). SNP summary statistics were extracted from GWAS results from two relevant cohorts: CONVERGE (China, Oxford and VCU Experimental Research on Genetic Epidemiology) and the Psychiatric Genetics Consortium study of Depression (PGC-MDD) representing 11139 Han Chinese (5647 female cases, 5492 controls) and 18663 European (9447 cases, 9215 controls) individuals, respectively. There were no genome-wide significant hits within an initial cross-population GWAS metaanalysis; however, after synchronizing phenotypic definitions between the two cohorts, a single, potentially sex-specific GWAS hit emerged on chr7, $24 \mathrm{~kb}$ upstream of the SLC29A4 gene [7p22.1; $P=6.2 \times 10^{-8}$; females-only recurrent MDD ( $>2$ episodes)]. Cross-population polygene score associations were assessed, with both the PGC-trained polygene score being significantly associated with the CONVERGE score $\left(P=1.65 \times 10^{-5}\right.$; recurrent MDD; $P$ value threshold $<0.4$; variance explained $<0.3 \%)$ and the CONVERGE-trained score being significantly associated with the PGC score $\left(P=8.82 \times 10^{-3}\right.$; females-only MDD; $P$-value threshold $<0.5$; variance explained $\sim 0.1 \%$ ). A binomial sign test showed a more consistent direction of effects with the PGC cohort in the CONVERGE cohort than expected by chance, with the most significant result at a $P$-value threshold of $0.05\left(P=2.4 \times 10^{-5}\right)$. Although no single variants remained genome-wide significant after multiple-testing correction for the number of GWAS performed, taken together, these results support a shared polygenic risk of MDD across populations. Overall, the study results support a complex etiology for MDD with potentially differing genetic factors across populations.

Dr Margarita Rivera-Sanchez (King's College London, UK) presented a study that examined a polymorphism in the FTO gene and its effect on BMI and depression (MDD). Both a high BMI (obesity) and MDD are major risk factors for chronic disease including type 2 diabetes, cardiovascular disease, and others. Following up from a study that showed an association between the rs9939609 polymorphism and BMI in MDD cases, but not controls (Rivera et al., 2012), the authors carried out a meta-analysis in 13701 individuals from five independent studies that had information on BMI and MDD and found an interaction between rs9939609, BMI, and MDD (fixed-effects: $\beta=0.13, P=3.087 \times 10^{-7}$; randomeffects: $\beta=0.12, P=0.027$ ). Effectively, patients with MDD showed an additional increase of $2.2 \%$ in their BMI for each rs9939609 risk allele beyond the effect of the rs 9939609 
genotype or MDD alone. This work has implications for understanding comorbidity factors and in turn predicting which MDD patients are at risk of high-BMI disorders.

Dr Panagiotis Ferentinos (University of Athens, Greece) introduced a study that investigated the familiality of important subphenotypes in MDD that have potential utility to address disease heterogeneity, AAO, and episode frequency (episodicity). The familiality was assessed in 1498 full siblings with recurrent (MDD) from the DeNt study. The SNP heritability of the same MDD subphenotypes was investigated in 3468 genotyped unrelated cases from the RADIANT and GSK-Munich studies using the GREML method. Significant familial clustering was found for both AAO and episodicity. The strength of the familial effect was measured with family-level intraclass correlation coefficients (ICC $=0.28$ for AAO, 0.07 for episodicity). The authors calculated from respective ICC estimates a maximal additive heritability of 0.56 for AAO and 0.14 for episodicity. The SNP heritability of AAO was $0.17(P=0.04)$; however, the analysis was underpowered for calculating the SNP heritability of episodicity.

\section{Autism: genomics and more (reported by Maria Tropeano and Joanna Martin)}

Dr Marta Biagioli (Massachusetts General Hospital, USA) described the transcriptional impact of the chromodomain helicase $C H D 8$ suppression in neural precursor cells. She identified 1756 genes differentially expressed after $C H D 8$ knockdown, most of which were upregulated. To distinguish between direct and indirect transcriptional effects, she also investigated the genome-wide sites of $C H D 8$ binding to chromatin from three different antibodies. She found 7324 replicated binding sites from all three antibodies; however, just $9.2 \%$ of the corresponding genes were differentially expressed after $C H D 8$ knockdown, which suggests that $C H D 8$ produces a much larger network of expression changes through indirect regulatory mechanisms. Genes directly downregulated by $C H D 8$ were enriched for genes involved in early fetal development, including chromatin modifiers and transcription factors; genes indirectly downregulated by $C H D 8$ were enriched for genes involved in neural development and ASD.

Dr Susan Santangelo (Maine Medical Center, USA) reviewed the genetic architecture of ASDs. She described autism as a clinically and genetically heterogeneous disorder with a significant genetic contribution from both common (SNPs) and rare genetic variants (CNVs, SNVs, LoF mutations, etc.). A number of these mutations are not specific for ASD, but they cross traditional diagnostic boundaries and are shared by different disorders (pleiotropy); as an example, she discussed the three genomewide hits shared by ASD and SCZ (TCF4, PCGEM1, MIR137), identified by the PGC cross-disorder analyses (Cross-Disorder Group of the Psychiatric Genomics Consortium et al., 2013a, 2013b). She also explained that autism is characterized by an extreme polygenicity, with many hundreds of genes involved, but little overlap is observed between genes implicated by common and rare variants; these hundreds of genes identified so far, however, seem to converge on a few common biological pathways involved in brain development, synapse function, and chromatin regulation. She concluded by emphasizing that biological factors involved in disease causation should be used to inform psychiatric nosological systems. 
Kaitlin Samocha (Massachusetts General Hospital, USA) presented an exome sequencing study of 3781 cases with an ASD (including 2297 cases from family trios) and 9937 controls. In an attempt to increase power for gene discovery, she used a Bayesian gene-based likelihood model named TADA (transmission and de-novo association) (He et al., 2013), which allows the combination of de-novo mutations with inherited variations and casecontrol information. She identified 33 genes with FDR less than 0.1 and 107 genes with 0.1 $\leq F D R<0.3$, with an excess of de-novo Lof mutations compared with inherited variants in both gene sets. She then refined TADA results by separating the two gene sets by the sex of the carrier (male : female in ASD $4: 1$ ). Genes with FDR less than0.1 showed significant enrichment of de-novo variants in female cases (female : male > 2; implied OR $\geq 20$ ); a modest enrichment of de-novo variants in females was also observed for genes with $0.1 \leq$ FDR $<0.3$ (female : male 1.3-1.4; implied OR 3-5). No significant difference between sexes was observed for inherited variants in both gene sets. It was noted that genes with 0.1 $\leq$ FDR $<0.3$ of moderate impact might contribute toward discrete subsets of ASD.

Dr Beate St. Pourcain (University of Bristol, UK) presented new data examining possible G $\times$ E correlations of maternal smoking during the first trimester of pregnancy and risk SNPs in relation to autistic traits (i.e. social communication difficulties) in children in the general population. Sixty-five independent SNPs that showed variance inequality for social communication problems at ages 8 and 11 years in the Avon Longitudinal Study of Parents and Children sample $(N \leq 5652)$ were selected for interaction analyses. St. Pourcain and colleagues tested whether each SNP showed an interaction effect with the presence of maternal smoking during pregnancy. An SNP within the RBFOX1 gene showed the strongest interaction effect for social communication traits at 8 years and maternal smoking in pregnancy. However, follow-up using a second sample, the UK Twins Early Development Study $(n=1330)$, which assessed autistic traits at age 8 years, did not replicate this finding, although this may be because of phenotype measurement differences. St. Pourcain concluded that non-additive effects (e.g. $\mathrm{G} \times \mathrm{E}$ interactions) may contribute toward the genetic architecture of autistic traits in the population, although the power to identify individual effects may be limited.

Dr Danielle Posthuma (Vrije Universiteit, the Netherlands) presented gene-set analyses using Joint Analysis of Genetic Variance software in trios from the Autism Genome Project $(n=2398)$ and the Autism Genetic Resource Exchange ( $n=1491)$. Previous pathway analyses (11 publications) have tended to utilize pathways available in public databases. Posthuma and colleagues analyzed expert-curated synaptic (17 gene sets) and glia-related (oligodendrocyte, astrocyte, microglia; 79 sets of genes) biological processes that were supported by empirical evidence (e.g. from animal knockout models). The results showed two significantly associated gene sets: microglial genes related to (a) cell proliferation and (b) cell locomotion and taxis, after accounting for multiple testing of pathways. Posthuma pointed out that microglia are the primary immune cells of the CNS and concluded that variation in genes related to certain microglial functions may contribute toward the pathophysiology of ASD. Work is ongoing to replicate these findings.

Dr Lauren Weiss (University of California San Francisco, USA) presented new data on CNVs in children with ASD, their relatives, as well as in control populations. Previous 
research suggests that females are protected from developing ASD (Robinson et al., 2013) and females who do develop ASD are more likely to have large, rare CNVs compared with affected males (Jacquemont et al., 2014). Dr Weiss presented data from three clinical ASD samples (Autism Genetic Resource Exchange, Autism Genome Project, Simons Simplex Collection) and two large control datasets (HapMap, 1000 Genomes). The results show an increased burden of large $(>30 \mathrm{~kb})$, rare $(<1 \%$ frequency) autosomal CNVs in females with ASD compared with affected males, in mothers compared with fathers of affected children, and also strikingly, in control females compared with control males in the population samples. In ASD children matched for number of CNVs, females had higher scores on the Social Responsiveness Scale than males. Furthermore, unaffected female siblings of ASD probands showed an enrichment of CNVs compared with male siblings. Dr Weiss suggested that the results may reflect an early developmental intolerance for CNVs in males, which may be associated with the higher rate of male fetal loss. Dr Weiss concluded by stressing the importance of controlling for sex or matching cases and controls for sex in genetic studies.

\section{Novel biostatistics and bioinformatics (reported by Gabriëlla Blokland and Lynsey Hall)}

Dr Robert Power (Institute of Psychiatry, King's College London, UK) investigated whether the heritability of cannabis use overlaps with the genetic predisposition to SCZ (Power et al., 2014). To determine how much of the variance in cannabis use $\left(r^{2}\right)$ in a general population sample can be explained by a SCZ PRS, the PGC-SCZ1 GWAS was used as the discovery sample. The PGC1 risk alleles and odds ratios were used to construct PRSs in an Australian twin sample consisting of $n=1011$ cannabis users and $n=1071$ nonusers. Cannabis userelated variables were 'use over lifetime: ever versus never', 'quantity of use', and 'age at initiation'. A higher genetic risk burden was associated with a greater likelihood of ever having used cannabis, and among those individuals who had used cannabis, it was associated with a higher quantity of cannabis use. The variance explained peaked at a risk scoring threshold of $P=0.1$. The association with SCZ risk was not limited to cannabis only, but appeared to extend to addiction in general. They found overlap with addiction for all substances (opiates, cocaine, amphetamines, alcoholism), except smoking, suggesting that it is a general predisposition. However, the direction of causation remains to be determined. As the majority of SCZ patients use cannabis and the SCZ risk scores include cannabis risk alleles, it could be that these cannabis risk alleles are actually predicting cannabis use. Using a discovery sample of non-cannabis-using schizophrenics might help to elucidate the causal mechanisms.

Dr Bjarni Vilhjálmsson (Harvard School of Public Health, USA, and Aarhus University, Denmark) explained how modeling LD increases the predictive accuracy of PRSs. LD between causal markers biases the effect estimates from GWAS. The standard approach of $P$-value thresholding association statistics, followed by LD pruning does not account for this bias, and yields suboptimal polygenic risk predictions. LD pruning lowers the heritability explained by the genotyped SNPs because you are throwing away markers that may have an effect, and ignores bias in the marginal least square estimate because of LD. This negative impact of LD on the prediction accuracy is expected to increase as GWAS sample sizes continue to grow. Vilhjálmsson and colleagues developed a Bayesian PRS method, LDpred, 
that estimates LD from a reference panel and reweights the effect estimates obtained from GWAS summary statistics to estimate posterior mean causal effect sizes, producing optimal PRS when model assumptions hold. These estimates have a closed-form solution under an infinitesimal prior where every marker is causal and effect sizes are drawn from a mean-zero normal distribution. Under a non-infinitesimal prior where only a fraction of markers are causal, estimates are approximated using a Markov Chain Monte Carlo approach, LDpred. When applied to simulated traits using real genotypes, LDpred outperforms existing methods (LD pruning + thresholding), particularly at large sample sizes. LDpred was applied to seven WTCCC diseases and considerable improvements in $r^{2}$ prediction accuracy were observed compared with standard PRS for all except one disease - for example, for type 1 diabetes, it improved from 30.4 to $37.8 \%$. When applied to PGC-SCZ2 GWAS summary statistics, using ISC as an independent validation sample, a significant improvement in prediction $r^{2}$ was observed as well: from15.7 to $18.3 \%$. The relative improvement in $r^{2}$ was even larger when predicting in samples of non-European descent: from 2.0 to $2.6 \%$. Although an improvement, LDpred is limited by how well the reference sample reflects the LD structure in the GWAS sample. If LD information from individual cohorts is available, the prediction accuracy of LDpred could improve even further.

Andrew Jaffe (Lieber Institute for Brain Development, USA) spoke about technological advances in RNA sequencing resolution and coverage permitting characterization of the transcription dynamics (Trapnell et al., 2010). He presented research analyzing the transcriptome at base-pair resolution in the DLPFC using DERfinder (differentially expressed region finder; Frazee et al., 2014). The study used 72 human samples across six important life stages - fetal (second trimester), infant, child, teen, adult, and elderly ( $n=6 /$ group). A total of 50650 differentially expression regions (DERs) were identified, agnostic of annotation, with significant and replicated expression changes across fetal and postnatal development. The majority of DERs had the highest expression levels in fetal life (81.7\%) and similar expression profiles of DERs were found across 16 diverse human brain regions and within the developing mouse cortex. He concluded that expression changes across development were being driven by a changing neuronal phenotype (Guintivano et al., 2013; Jaffe and Irizarry, 2014) related to differentiation and maturation, that the data highlighted conserved molecular signatures of transcriptional dynamics across brain development, and that annotation of the human brain transcriptome is incomplete.

Dale Nyholt (Department of Genetics and Computational Biology, Queensland Institute of Medical Research) presented research on using a novel gene-based approach to assess pleiotropy across the five major psychiatric disorders - ADHD, autism, BD, MDD, and SCZ - using summary data from the Cross-Disorder Group of the Psychiatric Genomics Consortium et al. (2013a, 2013b). He discussed the reasons why focusing on shared genes rather than shared SNPs may prove fruitful, namely, that genes are the predominant functional unit of the human genome, more closely related to biology, and gene-based tests incorporate allelic heterogeneity. The gene-based (GATES) test was implemented in the Fast ASsociation Tests (FAST) software (Chanda et al., 2013) and incorporated information on the $P$-value of the top SNP in each gene and the total number of effective, independent SNPs. Genetic overlap between disorders was assessed using a binomial test. Novel and significant overlaps were observed between ADHD and BD, and ADHD and autism, in 
addition to significant genetic overlap being observed between disorders that SNP-based tests had already indicated such as SCZ and BD, SCZ and major depression, SCZ and autism, and major depression and autism. He went on to present the results from subsequent pathway analysis for adult-onset disorders and between pairs of disorders. He concluded by reiterating the benefits of gene-based tests, stating that by combining gene-based association results across overlapping disorders, this method had identified numerous genes surpassing genome-wide significance, had extended previous findings from single SNP-based genetic overlap analysis (Cross-Disorder Group of the Psychiatric Genomics Consortium et al., 2013a, 2013b), and had provided insight into likely genes and biological mechanisms underlying the observed genetic correlation and comorbidity between these major psychiatric disorders.

Antonio Pardiñas (Cardiff University, Cardiff) compared the different available methodologies for addressing population structure in GWASs compared with PCA. The different methods that he discussed were spatial ancestry analysis (SPA) (Yang et al., 2012), which uses allele frequency as a function of geographic location, SNPWEIGHTS (Chen et al., 2013), which estimates principal components from large panels of individuals with known ancestry, and GAGA (Lao et al., 2014), which is based on analysis of molecular variance. The International Schizophrenia Consortium (International Schizophrenia Consortium et al., 2009) and CLOZUK (Rees et al., 2014) were used to test the corrective capabilities of these methods for samples with extensive and little population stratification, respectively. He discussed the results of these methods against other commonly used correction procedures, which involved PCA using EIGENSTRAT, Cochran-MantelHaenszel stratified analysis, and two mixed linear models computed with GCTA (Yang et al., 2011). The suitability of each method was assessed by observing how accurately the sample clustered and by measuring the inflation of the regression statistic using the lambda parameter (Devlin and Roeder, 1999). The conclusions that he drew were that PCA was generally applicable, SPA was good for extensively, but not less stratified populations, SNPWEIGHTS did not properly reflect the structure of samples because of the reference panels used, and that GAGA clusters were insufficient for adequate correction. He closed by stating that although mixed models could overcome these limitations, the most stringent procedures available also seem to suffer from overcorrection and power loss, as is consistent with recent discussions on their statistical properties (Yang et al., 2014).

\section{Acknowledgements}

This report was made possible by a grant from NIH R13AA017055. Each summary is the subjective understanding of the rapporteur for each session. The data reported are as heard during the presentation and where possible, all statements have been checked with the speaker for accuracy. However, the speakers are not responsible for any of the information contained in this report.

\section{References}

Agartz I, Brown AA, Rimol LM, Hartberg CB, Dale AM, Melle I, et al. (2011). Common sequence variants in the major histocompatibility complex region associate with cerebral ventricular size in schizophrenia. Biol Psychiatry 70:696-698. [PubMed: 21514568] 
Akpudo H, Aleksic B, Alkelai A, Burton C, Carrillo-Roa T, Chen DT, et al. (2014). Summaries of oral sessions at the XXI World Congress of Psychiatric Genetics, Boston, Massachusetts, 17-21 October 2013: state of the field. Psychiatr Genet 24:125-150. [PubMed: 24912047]

Alda M, Shao L, Wang JF, Lopez de Lara C, Jaitovich-Groisman I, Lebel V, et al. (2013). Alterations in phosphorylated cAMP response element-binding protein (pCREB) signaling: an endophenotype of lithium-responsive bipolar disorder? Bipolar Disord 15:824-831. [PubMed: 24238631]

Alkelai A, Baum A, Carless M, Crowley J, Dasbanerjee T, Dempster E, et al. (2008). The XVth World Congress of Psychiatric Genetics, October 7-11, 2007: rapporteur summaries of oral presentations. Am J Med Genet B Neuropsychiatr Genet 147B:233-277. [PubMed: 18297693]

Alzate AM, Benitez JG (2011). 'We are all depressed here'. Perceptions of suicide in a neighborhood in Ciudad Bolívar (Antioquia). Rev Fac Nac Salud Pública 29:251-255.

Amstadter AB, Balachandar V, Bergen SE, Ceulemans S, Christensen JH, Cole J, et al. (2010). Selected summaries from the XVII World Congress of Psychiatric Genetics, San Diego, California, USA, 4-8 November 2009. Psychiatr Genet 20:229-268. [PubMed: 20706171]

Anderson-Schmidt H, Beltcheva O, Brandon MD, Byrne EM, Diehl EJ, Duncan L, et al. (2013). Selected rapporteur summaries from the XX World Congress of Psychiatric Genetics, Hamburg, Germany, October 14-18, 2012. Am J Med Genet B Neuropsychiatr Genet 162B:96-121. [PubMed: 23341144]

Andreassen OA, Djurovic S, Thompson WK, Schork AJ, Kendler KS, O’Donovan MC, et al. (2013). Improved detection of common variants associated with schizophrenia by leveraging pleiotropy with cardiovascular-disease risk factors. Am J Hum Genet 92:197-209. [PubMed: 23375658]

Andreassen OA, Harbo HF, Wang Y, Thompson WK, Schork AJ, Mattingsdal M, et al. (2015). Genetic pleiotropy between multiple sclerosis and schizophrenia but not bipolar disorder: differential involvement of immune-related gene loci. Mol Psychiatry 20:207-214. [PubMed: 24468824]

Arking DE, Pulit SL, Crotti L, van der Harst P, Munroe PB, Koopmann TT, et al. (2014). Genetic association study of QT interval highlights role for calcium signaling pathways in myocardial repolarization. Nat Genet 46:826-836. [PubMed: 24952745]

Bagot RC, Zhang T, Wen X, Nguyen TTT, Nguyen H, Diorio J, et al. (2012). Variations in postnatal maternal care and the epigenetic regulation of metabotropic glutamate receptor 1 expression and hippocampal function in the rat. Proc Natl Acad Sci USA 16:109.

Baker K, Costain G, Fung WLA, Bassett AS (2014). Chromosomal microarray analysis - a routine clinical genetic test for patients with schizophrenia. Lancet Psychiatry 1:329-331. [PubMed: 26360988]

Baldessarini RJ, Tondo L, Floris G, Hennen J (2000). Effects of rapid cycling on response to lithium maintenance treatment in 360 bipolar I and II disorder patients. J Affect Disord 61:13-22. [PubMed: 11099736]

Barry G, Briggs JA, Vanichkina DP, Poth EM, Beveridge NJ, Ratnu VS, et al. (2014). The long noncoding RNA Gomafu is acutely regulated in response to neuronal activation and involved in schizophrenia-associated alternative splicing. Mol Psychiatry 19:486-494. [PubMed: 23628989]

Bedoya G, Montoya P, García J, Soto I, Bourgeois S, Carvajal L, et al. (2006). Admixture dynamics in Hispanics: a shift in the nuclear genetic ancestry of a South American population isolate. Proc Natl Acad Sci 103:7234-7239. [PubMed: 16648268]

Bergen S, Chen J, Dagdan E, Foon TS, Goes FS, Houlihan LM, et al. (2009). Selected summaries from the XVI World Congress of Psychiatric Genetics, Osaka, Japan, 11-15 October 2008. Psychiatr Genet 9:219-236.

Bergen SE, Balhara YP, Christoforou A, Cole J, Degenhardt F, Dempster E, et al. (2011). Summaries from the XVIII World Congress of Psychiatric Genetics, Athens, Greece, 3-7 October 2010. Psychiatr Genet 21:136-172. [PubMed: 21508788]

Berghofer A, Alda M, Adli M, Baethge C, Bauer M, Bschor T, et al. (2008). Longterm effectiveness of lithium in bipolar disorder: a multicenter investigation of patients with typical and atypical features. J Clin Psychiatry 69:1860-1868. [PubMed: 19026269]

Berndt A, Lee SY, Ramakrishnan C, Deisseroth K (2014). Structure-guided transformation of channelrhodopsin into a light-activated chloride channel. Science 344:420-424. [PubMed: 24763591] 
Bis JC, DeCarli C, Smith AV, van der Lijn F, Crivello F, Fornage M, et al. (2012). Common variants at $12 q 14$ and 12q24 are associated with hippocampal volume. Nat Genet 44:545-551. [PubMed: 22504421]

Boraska V, Franklin CS, Floyd JAB, Thornton LM, Huckins LM, Southam L, et al. (2014). A genomewide association study of anorexia nervosa. Mol Psychiatry 19:1085-1094. [PubMed: 24514567]

Borglum AD, Demontis D, Grove J, Pallesen J, Hollegaard MV, Pedersen CB, et al. (2014). Genomewide study of association and interaction with maternal cytomegalovirus infection suggests new schizophrenia loci. Mol Psychiatry 19:325-333. [PubMed: 23358160]

Bowden CL (2001). Clinical correlates of therapeutic response in bipolar disorder. J Affect Disord 67:257-265. [PubMed: 11869775]

Braff DL, Freeman R (2008). Clinically responsible genetic testing in neuropsychiatric patients: a bridge too far and too soon? Am J Psychiatry 165:952-955. [PubMed: 18676598]

Bralten J, Franke B, Waldman I, Rommelse N, Hartman C, Asherson P, et al. (2013). Candidate genetic pathways for attention-deficit/hyperactivity disorder (ADHD) show association to hyperactive/impulsive symptoms in children with ADHD. J Am Acad Child Adolesc Psychiatry 52:1204-1212. [PubMed: 24157394]

Bui ET, Anderson NK, Kassem L, McMahon FJ (2006). Do participants in genome sequencing studies of psychiatric disorders wish to be informed of their results? A survey study. J Genet Couns 15:77-83. [PubMed: 16761103]

Bulik CM, Sullivan PF, Tozzi F, Furberg H, Lichtenstein P, Pedersen NL (2006). Prevalence, heritability, and prospective risk factors for anorexia nervosa. Arch Gen Psychiatry 63:305-312. [PubMed: 16520436]

Bulik-Sullivan B, Loh P-R, Finucane H, Ripke S, Yang J. Psychiatric Genomics Consortium, et al. (2015). LD score regression distinguishes confounding from polygenicity in genome-wide association studies. Nat Genet 47:291-295. [PubMed: 25642630]

Burke W, Matheny Antommaria AH, Bennett R, Botkin J, Clayton EW, Henderson GE, et al. (2013). Recommendations for returning genomic incidental findings? We need to talk!. Genet Med 15:854-859. [PubMed: 23907645]

Buxton JL, Suderman M, Pappas JJ, Borghol N, McArdle W, Blakemore AI, et al. (2014). Human leukocyte telomere length is associated with DNA methylation levels in multiple subtelomeric and imprinted loci. Sci Rep 4:4954. [PubMed: 24828261]

Byrne EM, Carrillo-Roa T, Penninx BW, Sallis HM, Viktorin A, Chapman B, et al. (2014). Applying polygenic risk scores to postpartum depression. Arch Womens Ment Health 17:519-528. [PubMed: 25037970]

Calabrese JR, Fatemi SH, Kujawa M, Woyshville MJ (1996). Predictors of response to mood stabilizers. J Clin Psychopharmacol 16:24S-31S. [PubMed: 8707997]

Calvo AC, Scherer T, Pey AL, Ying M, Winge I, McKinney J, et al. (2010). Effect of pharmacological chaperones on brain tyrosine hydroxylase and tryptophan hydroxylase 2. J Neurochem 114:853863. [PubMed: 20492352]

Campos-Sánchez R, Raventós H, Barrantes R (2013). Ancestry informative markers clarify the regional admixture variation in the Costa Rican population. Hum Biol 85:721-740. [PubMed: 25078957]

Carter SL, Cibulskis K, Helman E, Mckenna A, Shen H, Zack T, et al. (2012). Absolute quantification of somatic DNA alterations in human cancer. Nat Biotech 30:413-421.

Cawthon RM, Smith KR, O'Brien E, Sivatchenko A, Kerber RA (2003). Association between telomere length in blood and mortality in people aged 60 years or older. Lancet 361:393-395. [PubMed: 12573379]

Champagne FA, Weaver IC, Diorio J, Dymov S, Szyf M, Meaney MJ (2006). Maternal care associated with methylation of the estrogen receptor-alphalb promoter and estrogen receptor-alpha expression in the medial preoptic area of female offspring. Endocrinology 147:2909-2915. [PubMed: 16513834]

Chanda P, Huang H, Arking DE, Bader JS (2013). Fast association tests for genes with FAST. PLoS One 8:e68585. [PubMed: 23935874] 
Chen CY, Pollack S, Hunter DJ, Hirschhorn JN, Kraft P, Price AL (2013). Improved ancestry inference using weights from external reference panels. Bioinformatics 29:1399-1406. [PubMed: 23539302]

Cho I, Yamanishi S, Cox L, Methé BA, Zavadil J, Li K, et al. (2012). Antibiotics in early life alter the murine colonic microbiome and adiposity. Nature 488:621-626. [PubMed: 22914093]

Chung K, Wallace J, Kim S-Y, Kalyanasundaram S, Andalman AS, Davidson TJ, et al. (2013). Structural and molecular interrogation of intact biological systems. Nature 497:332-337. [PubMed: 23575631]

Codd V, Nelson CP, Albrecht E, Mangino M, Deelen J, Buxton JL, et al. (2013). Identification of seven loci affecting mean telomere length and their association with disease. Nat Genet 45:422-427. [PubMed: 23535734]

Cross-Disorder Group of the Psychiatric Genomics Consortium, Lee Sh, Ripke S, Neale BM, Faraone SV, Purcell SM, et al. (2013a). Genetic relationship between five psychiatric disorders estimated from genome-wide SNPs. Nat Genet 45:984-994. [PubMed: 23933821]

Cross-Disorder Group of the Psychiatric Genomics Consortium, Smoller JW, Craddock N, Kendler K, Lee PH, Neale BM, et al. (2013b). Identification of risk loci with shared effects on five major psychiatric disorders: a genome-wide analysis. Lancet 381:1371-1379. [PubMed: 23453885]

Cruceanu C, Alda M, Grof P, Rouleau GA, Turecki G (2012). Synapsin II is involved in the molecular pathway of lithium treatment in bipolar disorder. Plos One 7:e32680. [PubMed: 22384280]

Dai N, Foldager L, Gallego JA, Hack LM, Ji Y, Lett TA, et al. (2012). Summaries from the XIX World Congress of Psychiatric Genetics, Washington, DC, September 10-14, 2011. Am J Med Genet B Neuropsychiatr Genet 159B:128-129. [PubMed: 22180335]

De Oliveira CM, Pereira AC, De Andrade M, Soler JM, Krieger JE (2008). Heritability of cardiovascular risk factors in a Brazilian population: Baependi Heart Study. BMC Med Genet 9:32. [PubMed: 18430212]

Deisseroth K (2011). Optogenetics. Nat Methods 8:26-29. [PubMed: 21191368]

Devlin B, Roeder K (1999). Genomic control for association studies. Biometrics 55:997-1004. [PubMed: 11315092]

Dunlop BW, Binder EB, Cubells JF, Goodman MM, Kelley ME, Kinkead B, et al. (2012). Predictors of remission in depression to individual and combined treatments (PReDICT): study protocol for a randomized controlled trial. Trials 13:106. [PubMed: 22776534]

Estrada JA, Contreras I, Pliego-Rivero FB, Otero GA (2014). Molecular mechanisms of cognitive impairment in iron deficiency: alterations in brain-derived neurotrophic factor and insulin-like growth factor expression and function in the central nervous system. Nutr Neurosci 17:193-206. [PubMed: 24074845]

Fears SC, Service SK, Kremeyer B, Araya C, Araya X, Bejarano J, et al. (2014). Multisystem component phenotypes of bipolar disorder for genetic investigations of extended pedigrees. JAMA Psychiatry 71:375-387. [PubMed: 24522887]

Fejgin K, Nielsen J, Birknow MR, Bastlund JF, Nielsen V, Lauridsen JB, Stefansson H (2013). A mouse model that recapitulates cardinal features of the 15q13.3 microdeletion syndrome including schizophrenia- and epilepsy-related alterations. Biol Psychiatry 76:128-137. [PubMed: 24090792]

Fossbakk A, Kleppe R, Knappskog PM, Martinez A, Haavik J (2014). Functional studies of tyrosine hydroxylase missense variants reveal distinct patterns of molecular defects in Dopa-responsive dystonia. Hum Mutat 35:880-890. [PubMed: 24753243]

Frazee AC, Sabunciyan S, Hansen KD, Irizarry RA, Leek JT (2014). Differential expression analysis of RNA-seq data at single-base resolution. Biostatistics 15:413-426. [PubMed: 24398039]

Fromer M, Pocklington AJ, Kavanagh DH, Williams HJ, Dwyer S, Gormley P, et al. (2014). De novo mutations in schizophrenia implicate synaptic networks. Nature 506:179-184. [PubMed: 24463507]

Garriock HA, Kraft JB, Shyn SI, Peters EJ, Yokoyama JS, Jenkins GD, et al. (2010). A genomewide association study of citalopram response in major depressive disorder. Biol Psychiatry 67:133138. [PubMed: 19846067]

Gauthier J, Champagne N, Lafrenière RG, Xiong L, Spegelman D, Brustein E, et al. (2010). De novo mutations in the gene encoding the synaptic scaffolding protein SHANK3 in patients ascertained for schizophrenia. Proc Natl Acad Sci USA 107:7863-7868. [PubMed: 20385823] 
Gelernter J, Sherva R, Koesterer R, Almasy L, Zhao H, Kranzler HR, Farrer L (2014). Genome-wide association study of cocaine dependence and related traits: FAM53B identified as a risk gene. Mol Psychiatry 19:717-723. [PubMed: 23958962]

Gershon E, Alliey-Rodriguez N (2013). New ethical issues for genetic counseling in common mental disorders. Am J Psychiatry 170:968-976. [PubMed: 23897273]

Glahn DC, Almasy L, Barguil M, Hare E, Peralta JM, Kent JW, et al. (2010). Neurocognitive endophenotypes for bipolar disorder identified in multiplex multigenerational families. Arch Gen Psychiatry 67:168-177. [PubMed: 20124116]

Golding J, Pembrey M, Jones R (2001). ALSPAC - the avon longitudinal study of parents and children. Paediatr Perinat Epidemiol 15:74-87. [PubMed: 11237119]

Golzio C, Willer J, Talkowski ME, Oh EC, Taniguchi Y, Jacquemont S, et al. (2012). KCTD13 is a major driver of mirrored neuroanatomical phenotypes of the $16 \mathrm{p} 11.2$ copy number variant. Nature 485:363-367. [PubMed: 22596160]

Gonzalez S, Xu C, Ramirez M, Zavala J, Armas R, Contreras SA, et al. (2013). Suggestive evidence for association between L-type voltage-gated calcium channel (CACNA1C) gene haplotypes and bipolar disorder in Latinos: a family-based association study. Bipolar Disord 15:206-214. [PubMed: 23437964]

Gonzalez S, Camarillo C, Rodriguez M, Ramirez M, Zavala J, Armas R, et al. (2014). A genome-wide linkage scan of bipolar disorder in Latino families identifies susceptibility loci at 8q24 and 14q32. Am J Med Genet Part B 165:479-491.

Green RC, Berg JS, Grody WW, Kalia SM, Korf BR, Martin CL, et al. (2013). ACMG recommendations for reporting of incidental findings in clinical exome and genome sequencing. Genet Med 15:565-574. [PubMed: 23788249]

Greil W, Ludwig-Mayerhofer W, Erazo N, Schochlin C, Schmidt S, Engel RR, et al. (1997). Lithium versus carbamazepine in the maintenance treatment of bipolar disorders - a randomised study. $\mathrm{J}$ Affect Disord 43:151-161. [PubMed: 9165384]

Grof P, Alda M, Grof E, Fox D, Cameron P (1993). The challenge of predicting response to stabilising lithium treatment. The importance of patient selection. Br J Psychiatry Suppl 21:16-19.

Grof P, Duffy A, Cavazzoni P, Grof E, Garnham J, MacDougall M, et al. (2002). Is response to prophylactic lithium a familial trait? J Clin Psychiatry 63:942-947. [PubMed: 12416605]

Guintivano J, Aryee MJ, Kaminsky ZA (2013). A cell epigenotype specific model for the correction of brain cellular heterogeneity bias and its application to age, brain region and major depression. Epigenetics 8:290-302. [PubMed: 23426267]

Gunaydin LA, AGrosenick L, Finkelstein JC, Kauvar IV, Fenno LE, Adhikari A, et al. (2014). Natural neural projection dynamics underlying social behavior. Cell 157:1535-1551. [PubMed: 24949967]

Hare E, Glahn DC, Dassori A, Raventos H, Nicolini H, Ontiveros A, et al. (2010). Heritability of age of onset of psychosis in schizophrenia. Am J Med Genet Part B 153B:298-302. [PubMed: 19350535]

Hart AB, Engelhardt BE, Wardle MC, Sokoloff G, Stephens M, de Wit H, et al. (2012). Genome-wide association study of d-amphetamine response in healthy volunteers identifies putative associations, including cadherin 13 (CDH13). PLoS One 7:e42646. [PubMed: 22952603]

He X, Sanders SJ, Liu L, De Rubeis S, Lim ET, Sutcliffe JS, et al. (2013). Integrated model of de novo and inherited genetic variants yields greater power to identify risk genes. PLoS Genet 9:e1003671. [PubMed: 23966865]

Hicks JK, Swen JJ, Thorn CF, Sangkuhl K, Kharasch ED, Ellingrod VL, et al. (2013). Clinical Pharmacogenetics Implementation Consortium guideline for CYP2D6 and CYP2C19 genotypes and dosing of tricyclic antidepressants. Clin Pharmacol Ther 93:402-408. [PubMed: 23486447]

Hill WG, Goddard ME, Visscher PM (2008). Data and theory point to mainly additive genetic variance for complex traits. PLoS Genet 4:e1000008. [PubMed: 18454194]

Hinney A, Scherag A, Jarick I, Albayrak Ö, Pütter C, Pechlivanis S, et al. (2011). Genome-wide association study in German patients with attention deficit/hyperactivity disorder. Am J Med Genet Part B Neuropsychiatr Genet 156B:888-897.

Howland RH (2008). Sequenced treatment alternatives to relieve depression (STAR*D). Part 2: Study outcomes. J Psychosoc Nurs Ment Health Serv 46:21-24. 
Inglis A, Koehn D, McGillivray B, Stewart SE, Austin J (2015). Evaluating a unique, specialist psychiatric genetic counseling clinic: uptake and impact. Clin Genet 87:218-224. [PubMed: 24773225]

International Parkinson Disease Genomics Consortium, Nalls MA, Plagnol V, Hernandez DG, Sharma M, Sheerin UM, et al. (2011). Imputation of sequence variants for identification of genetic risks for Parkinson's disease: a meta-analysis of genome-wide association studies. Lancet 377:641-649. [PubMed: 21292315]

International Schizophrenia Consortium, Purcell SM, Wrasy NR, Stone JL, Visscher PM, O'Donovan MC, et al. (2009). Common polygenic variation contributes to risk of schizophrenia and bipolar disorder. Nature 460:748-752. [PubMed: 19571811]

Iossifov I, Ronemus M, Levy D, Wang Z, Hakker I, Rosenbaum J, et al. (2012). De novo gene disruptions in children on the autistic spectrum. Neuron 74:285-299. [PubMed: 22542183]

Ising M, Lucae S, Binder EB, Bettecken T, Uhr M, Ripke S, et al. (2009). A genomewide association study points to multiple loci that predict antidepressant drug treatment outcome in depression. Arch Gen Psychiatry 66:966-975. [PubMed: 19736353]

Jacquemont S, Reymond A, Zufferey F, Harewood L, Walters RG, Kutalik Z, et al. (2011). Mirror extreme BMI phenotypes associated with gene dosage at the chromosome 16p11.2 locus. Nature 478:97-102. [PubMed: 21881559]

Jacquemont S, Coe BP, Hersch M, Duyzend MH, Krumm N, Bergmann S, et al. (2014). A higher mutational burden in females supports a 'female protective model' in neurodevelopmental disorders. Am J Hum Genet 94:415-425. [PubMed: 24581740]

Jaffe AE, Irizarry RA (2014). Accounting for cellular heterogeneity is critical in epigenome-wide association studies. Genome Biol 15:R31. [PubMed: 24495553]

Jahanshad N, Kochunov PV, Sprooten E, Mandl RC, Nichols TE, Almasy L, et al. (2013). Multi-site genetic analysis of diffusion images and voxelwise heritability analysis: a pilot project of the ENIGMA-DTI working group. Neuroimage 81:455-469. [PubMed: 23629049]

Jennings JH, Rizzi G, Stamatakis AM, Ung RL, Stuber GD (2013). The inhibitory circuit architecture of the lateral hypothalamus orchestrates feeding. Science 341:1517-1521. [PubMed: 24072922]

Jensen AB, Moseley PL, Oprea TI, Ellesøe SG, Eriksson R, Schmock H, et al. (2014). Temporal disease trajectories condensed from population-wide registry data covering 6.2 million patients. Nat Commun 5:4022. [PubMed: 24959948]

Ji Y, Hebbring S, Zhu H, Jenkins GD, Biernacka J, Snyder K, et al. (2011). Glycine and a glycine dehydrogenase (GLDC) SNP as citalopram/escitalopram response biomarkers in depression: pharmacometabolomics-informed pharmacogenomics. Clin Pharmacol Ther 89:97-104. [PubMed: 21107318]

Ji Y, Biernacka JM, Hebbring S, Chai Y, Jenkins GD, Batzler A, et al. (2013). Pharmacogenomics of selective serotonin reuptake inhibitor treatment for major depressive disorder: genome-wide associations and functional genomics. Pharmacogenomics J 13:456-463. [PubMed: 22907730]

Ji Y, Schaid DJ, Desta Z, Kubo M, Batzler AJ, Snyder K, et al. (2014). Citalopram and escitalopram plasma drug and metabolite concentrations: genome-wide associations. Br J Clin Pharmacol 78:373-383. [PubMed: 24528284]

Karayiorgou M, Morris MA, Morrow B, Chprintzen RJ, Goldberg R, Borrow J, et al. (1995). Schizophrenia susceptibility associated with interstitial deletions of chromosome 22q11. Proc Natl Acad Sci USA 92:7612-7616. [PubMed: 7644464]

Kato T, Ishiwata M, Mori K, Washizuka S, Tajima O, Akiyama T, Kato N (2003). Mechanisms of altered $\mathrm{Ca}^{2}+$ signalling in transformed lymphoblastoid cells from patients with bipolar disorder. Int J Neuropsychopharmacol 6:379-389. [PubMed: 14604453]

Kato HE, Zhang F, Yizhar O, Ramakrishnan C, Nishizawa, Hirata K, et al. (2012). Crystal structure of the channelrhodopsin light-gated cation channel. Nature 482:369-374. [PubMed: 22266941]

Kim S-Y, Adhikari A, Lee SY, Marshel JH, Kim CK, Mallory CS, et al. (2013). Diverging neural pathways assemble a behavioural state from separable features in anxiety. Nature 496:219-223. [PubMed: 23515158] 
Kirov G, Pocklington AJ, Holmans P, Ivanov D, Ikeda M, Ruderfer D, et al. (2012). De novo CNV analysis implicates specific abnormalities of postsynaptic signalling complexes in the pathogenesis of schizophrenia. Mol Psychiatry 17:142-153. [PubMed: 22083728]

Klein C, Westenberger A (2012). Genetics of Parkinson's disease. Cold SpringHarb Perspect Med 2:a008888.

Klengel T, Mehta D, Anacker C, Rex-Haffner M, Pruessner JC, Pariante CM, et al. (2013). Allelespecific FKBP5 DNA demethylation mediates gene-childhood trauma interactions. Nat Neurosci 16:33-41. [PubMed: 23201972]

Kochunov P, Jahanshad N, Sprooten E, Nichols TE, Mandl RC, Almasy L, et al. (2014). Multi-site study of additive genetic effects on fractional anisotropy of cerebral white matter: comparing meta and megaanalytical approaches for data pooling. Neuroimage 95:136-150. [PubMed: 24657781]

Kong A, Frigge ML, Masson G, Besenbacher S, Sulem P, Magnusson G, et al. (2012). Rate of de novo mutations and the importance of father's age to disease risk. Nature 488:471-475. [PubMed: 22914163]

Kruger S, Alda M, Young LT, Goldapple K, Parikh S, Mayberg HS (2006). Risk and resilience markers in bipolar disorder: brain responses to emotional challenge in bipolar patients and their healthy siblings. Am J Psychiatry 163:257-264. [PubMed: 16449479]

Lao O, Liu F, Wollstein A, Kayser M (2014). GAGA: a new algorithm for genomic inference of geographic ancestry reveals fine level population substructure in Europeans. PLoS Comput Biol 10:e1003480. [PubMed: 24586132]

Le Chatelier E, Nielsen T, Qin J, Prifti E, Hildebrand F, Falony G, et al. (2013). Richness of human gut microbiome correlates with metabolic markers. Nature 500:541-546. [PubMed: 23985870]

Leblond CS, Nava C, Polge A, Gauthier J, Huguet G, Lumbroso S, et al. (2014). Meta-analysis of SHANK Mutations in Autism Spectrum Disorders: a gradient of severity in cognitive impairments. PLoS Genet 10:e1004580. [PubMed: 25188300]

Lencz T, Guha S, Liu C, Rosenfeld J, Mukherjee S, DeRosse P, et al. (2013). Genome-wide association study implicates NDST3 in schizophrenia and bipolar disorder. Nat Commun 4:2739. [PubMed: 24253340]

Lencz T, Knowles E, Davies G, Guha S, Liewald DC, Starr JM, et al. (2014). Molecular genetic evidence for overlap between general cognitive ability and risk for schizophrenia: a report from the Cognitive Genomics consorTium (COGENT). Mol Psychiatry 19:168-174. [PubMed: 24342994]

Leon PE, Raventos H, Lynch E, Morrow J, King MC (1992). The gene for an inherited form of deafness maps to chromosome 5q31. Proc Natl Acad Sci USA 89:5181-5184. [PubMed: 1350680]

Li J, Jia H, Cai X, Zhong H, Feng Q, Sunagawa S, et al. (2014). An integrated catalog of reference genes in the human gut microbiome. Nat Biotechnol 32:834-841. [PubMed: 24997786]

Lim ET, Wurtz P, Havulinna AS, Palta P, Tukiainen T, Rehnstrom K, et al. (2014). Distribution and medical impact of loss-of-function variants in the Finnish founder population. PLoS Genet 10:e1004494. [PubMed: 25078778]

Liou AP, Turnbaugh PJ (2012). Antibiotic exposure promotes fat gain. Cell Metab 16:408-410. [PubMed: 23040064]

Lo WS, Lau CF, Xuan Z, Chan CF, Feng GY, He L, et al. (2004). Association of SNPs and haplotypes in GABAA receptor beta2 gene with schizophrenia. Mol Psychiatry 9:603-608. [PubMed: 14699426]

Lo WS, Harano M, Gawlik M, Yu Z, Chen J, Pun FW, et al. (2007a). GABRB2 association with schizophrenia: commonalities and differences between ethnic groups and clinical subtypes. Biol Psychiatry 61:653-660. [PubMed: 16950232]

Lo WS, Xu Z, Yu Z, Pun FW, Ng SK, Chen J, et al. (2007b). Positive selection within the schizophrenia-associated GABA(A) receptor beta(2) gene. Plos One 2:e462. [PubMed: 17520021] 
Lobo DSS, Quilty LC, Martins SS, Tavares H, Vallada H, Kennedy JL, et al. (2014). Pathological gambling subtypes: a comparison of treatment-seeking and non-treatment-seeking samples from Brazil and Canada. Addict Behav 39:1172-1175. [PubMed: 24727115]

Lopez de Lara C, Jaitovich-Groisman I, Cruceanu C, Mamdani F, Lebel V, Yerko V, et al. (2010). Implication of synapse-related genes in bipolar disorder by linkage and gene expression analyses. Int J Neuropsychopharmacol 13:1397-1410. [PubMed: 20667171]

Lundby A, Rossin EJ, Steffensen AB, Acha MR, Newton-Cheh C, Pfeufer A, et al. (2014). Annotation of loci from genome-wide association studies using tissue-specific quantitative interaction proteomics. Nat Methods 11:868-874. [PubMed: 24952909]

Magalhaes Da Silva T, Sandhya Rani MR, De Oliveira Costa GN, Figueiredo MA, Melo PS, Nascimento JF, et al. (2015). The correlation between ancestry and color in two cities of Northeast Brazil with contrasting ethnic compositions. Eur J Hum Genet 23:984-989. [PubMed: 25293718]

Malhotra D, Sebat J (2012). CNVs: harbingers of a rare variant revolution in psychiatric genetics. Cell 148:1223-1241. [PubMed: 22424231]

Malkus KA, Tsika E, Ischiropoulos H (2009). Oxidative modifications, mitochondrial dysfunction, and impaired protein degradation in Parkinson's disease: how neurons are lost in the Bermuda triangle. Mol Neurodegener 4:24. [PubMed: 19500376]

Mamdani F, Alda M, Grof P, Young LT, Rouleau G, Turecki G (2008). Lithium response and genetic variation in the CREB family of genes. Am J Med Genet B Neuropsychiatr Genet 147B:500 504. [PubMed: 18189280]

Manchia M, Adli M, Akula N, Ardau R, Aubry JM, Backlund L, et al. (2013). Assessment of response to lithium maintenance treatment in bipolar disorder: a Consortium on Lithium Genetics (ConLiGen) report. Plos One 8:e65636. [PubMed: 23840348]

Manolio TA, Collins FS, Cox NJ, Goldstein DB, Hindorff LA, Hunter DJ, et al. (2009). Finding the missing heritability of complex diseases. Nature 461:747-753. [PubMed: 19812666]

Marballi K, Mccullumsmith R, Yates S, Escamilla M, Leach R, Raventos H, et al. (2014). Global signaling effects of a schizophrenia-associated missense mutation in neuregulin 1: an exploratory study using whole genome and novel kinome approaches. J Neural Transm 121:479-490. [PubMed: 24380930]

Márquez C, Poirier GL, Cordero MI, Larsen MH, Groner A, Marquis J, et al. (2013). Peripuberty stress leads to abnormal aggression, altered amygdala and orbitofrontal reactivity and increased prefrontal MAOA gene expression. Transl Psychiatry 3:e216. [PubMed: 23321813]

Martinsson L, Wei Y, Xu D, Melas PA, Mathé AA, Schalling M, et al. (2013). Long-term lithium treatment in bipolar disorder is associated with longer leukocyte telomeres. Transl Psychiatry 3:e261. [PubMed: 23695236]

McGowan PO, Sasaki A, D’Alessio AC, Dymov S, Labonte B, Szyf M, et al. (2009). Epigenetic regulation of the glucocorticoid receptor in human brain associates with childhood abuse. Nat Neurosci 12:342-348. [PubMed: 19234457]

Mcinnes LA, Service SK, Reus VI, Barnes G, Charlat O, Jawahar S, et al. (2001). Fine-scale mapping of a locus for severe bipolar mood disorder on chromosome 18p11.3 in the Costa Rican population. Proc Natl Acad Sci USA 98:11485-11490. [PubMed: 11572994]

Meaney MJ, Szyf M (2005). Environmental programming of stress responses through DNA methylation: life at the interface between a dynamic environment and a fixed genome. Dialogues Clin Neurosci 7:103-123. [PubMed: 16262207]

Mehta D, Newport DJ, Frishman G, Kraus L, Rex-Haffner M, Ritchie JC, et al. (2014). Early predictive biomarkers for postpartum depression point to a role for estrogen receptor signaling. Psychol Med 5:1-14.

Montiel-Castro AJ, González-Cervantes RM, Bravo-Ruiseco G, Pacheco-López G (2013). The microbiota-gut-brain axis: neurobehavioral correlates, health and sociality. Front Integr Neurosci 7:70. [PubMed: 24109440]

Mors O, Perto GP, Mortensen PB (2011). The Danish Psychiatric CentralResearch Register. Scand J Public Health 39:54-57. [PubMed: 21775352] 
Mühleisen TW, Leber M, Schulze TG, Strohmaier J, Degenhardt F, Treutlein J, et al. (2014). Genomewide association study reveals two new risk loci for bipolar disorder. Nat Commun 5:3339. [PubMed: 24618891]

Neale BM, Lasky-Su J, Anney R, Franke B, Zhou K, Maller JB, et al. (2008). Genome-wide association scan of attention deficit hyperactivity disorder. Am J Med Genet B Neuropsychiatr Genet 147B:1337-1344. [PubMed: 18980221]

Neale BM, Medland SE, Ripke S, Asherson P, Franke B, Lesch K-PP, et al. (2010). Meta-analysis of genome-wide association studies of attention-deficit/hyperactivity disorder. J Am Acad Child Adolesc Psychiatry 49:884-897. [PubMed: 20732625]

Ng SK, Lo WS, Pun FW, Zhao C, Yu Z, Chen J, et al. (2010). A recombination hotspot in a schizophrenia-associated region of GABRB2. Plos One 5:0009547.

Overstreet DH, Griebel G (2005). Antidepressant-like effects of the vasopressin V1b receptor antagonist SSR149415 in the Flinders Sensitive Line rat. Pharmacol Biochem Behav 82:223227. [PubMed: 16181667]

Parikshak NN, Luo R, Zhang A, Won H, Lowe JK, Chandran V, et al. (2013). Integrative functional genomic analyses implicate specific molecular pathways and circuits in autism. Cell 155:10081021. [PubMed: 24267887]

Park A, Stacy M (2009). Non-motor symptoms in Parkinson's disease. J Neurol 256:293-298. [PubMed: 19711119]

Passmore MJ, Garnham J, Duffy A, MacDougall M, Munro A, Slaney C, et al. (2003). Phenotypic spectra of bipolar disorder in responders to lithium versus lamotrigine. Bipolar Disord 5:110 114. [PubMed: 12680900]

Penninx BW, Beekman AT, Smit JH, Zitman FG, Nolen WA, Spinhoven P, et al. (2008). The Netherlands Study of Depression and Anxiety (NESDA): rationale, objectives and methods. Int J Methods Psychiatr Res 17:121-140. [PubMed: 18763692]

Penninx BW, Milaneschi Y, Lamers F, Vogelzangs N (2013). Understanding the somatic consequences of depression: biological mechanisms and the role of depression symptom profile. BMC Med 11:129. [PubMed: 23672628]

Petrovski S, Wang Q, Heinzen EL, Allen AS, Goldstein DB (2013). Genic intolerance to functional variation and the interpretation of personal genomes. PLoS Genet 9:e1003709. [PubMed: 23990802]

Petryshen TL, Middleton FA, Tahl AR, Rockwell GN, Purcell S, Aldinger KA, et al. (2005). Genetic investigation of chromosome 5q GABAA receptor subunit genes in schizophrenia. Mol Psychiatry 10:1074-1088. 1057. [PubMed: 16172613]

Philibert RA, Sandhu H, Hollenbeck NH, Gunter T, Adams W, Madan A (2008). The relationship of 5HTT (SLC6A4) methylation and genotype on mRNA expression and liability to major depression and alcohol dependence in subjects from the Iowa Adoption Studies. Am J Med Genet B Neuropsychiatr Genet 147B:543-549. [PubMed: 17987668]

Pidsley R, Viana J, Hannon E, Spiers H, Troakes C, Al-Saraj S, et al. (2014). Methylomic profiling of human brain tissue supports a neurodevelopmental origin for schizophrenia. Genome Biol 15:483. [PubMed: 25347937]

Pineda D, Lopera F, Puerta I, Trujillo-Orrego N, Aguirre-Acevedo D, Hincapié-Henao L, et al. (2011). Potential cognitive endophenotypes in multi-generational families: segregating ADHD from a genetic isolate. Atten Defic Hyperact Disord 3:291-299. [PubMed: 21779842]

Polymeropoulos MH, Higgins JJ, Golbe LI, Johnson WG, Ide SE, Di Iorio G, et al. (1996). Mapping of a gene for Parkinson's disease to chromosome 4q21-q23. Science 274:1197-1199. [PubMed: 8895469]

Porcelli S, Fabbri C, Serretta A (2012). Meta-analysis of serotonin transporter gene promoter polymorphism (5-HTTLPR) association with antidepressant efficacy. Eur Neuropsychopharmacol 22:239-258. [PubMed: 22137564]

Poultney CS, Goldberg AP, Drapeau E, Kou Y, Harony-Nicolas H, Kajiwara Y, et al. (2013). Identification of small exonic CNV from whole-exome sequence data and application to autism spectrum disorder. Am J Hum Genet 93:607-619. [PubMed: 24094742] 
Power RA, Verweij KJ, Zuhair M, Montgomery GW, Henders AK, Heath AC, et al. (2014). Genetic predisposition to schizophrenia associated with increased use of cannabis. Mol Psychiatry 19:1201-1204. [PubMed: 24957864]

Psychiatric GWAS Consortium Bipolar Disorder Working Group (2011). Large-scale genome-wide association analysis of bipolar disorder identifies a new susceptibility locus near ODZ4. Nat Genet 43:977-983. [PubMed: 21926972]

Pun FW, Zhao C, Lo WS, Ng SK, Tsang SY, Nimgaonkar V, et al. (2011). Imprinting in the schizophrenia candidate gene GABRB2 encoding GABA(A) receptor beta(2) subunit. Mol Psychiatry 16:557-568. [PubMed: 20404824]

Purcell SM, Moran JL, Fromer M, Ruderfer D, Solovieff N, Roussos P, et al. (2014). A polygenic burden of rare disruptive mutations in schizophrenia. Nature 506:185-190. [PubMed: 24463508]

Rees E, Walters JT, Georgieva L, Isles AR, Chambert KD, Richards AL, et al. (2014). Analysis of copy number variations at 15 schizophrenia-associated loci. Br J Psychiatry 204:108-114. [PubMed: 24311552]

Révész D, Verhoeven JE, Milaneschi Y, de Geus EJ, Wolkowitz OM, Penninx BW (2014). Dysregulated physiological stress systems and accelerated cellular aging. Neurobiol Aging 35:1422-1430. [PubMed: 24439483]

Rietveld CA, Medland SE, Derringer J, Yang J, Esko T, Martin NW, et al. (2013). GWAS of 126,559 individuals identifies genetic variants associated with educational attainment. Science 340:14671471. [PubMed: 23722424]

Ripke S, Sanders AR, Kendler KS, Levinson DF, Sklar P, Holmans PA, et al. (2011). Genome-wide association study identifies five new schizophrenia loci. Nat Genet 43:969-976. [PubMed: 21926974]

Ripke S, O’Dushlaine C, Chambert K, Moran JL, Kahler AK, Akterin S, et al. (2013). Genome-wide association analysis identifies 13 new risk loci for schizophrenia. Nat Genet 45:1150-1159. [PubMed: 23974872]

Rivera M, Cohen-Woods S, Kapur K, Breen G, Ng MY, Butler AW, et al. (2012). Depressive disorder moderates the effect of the FTO gene on body mass index. Mol Psychiatry 17:6.

Robins E, Guze SB (1970). Establishment of diagnostic validity in psychiatric illness: its application to schizophrenia. Am J Psychiatry 126:983-987. [PubMed: 5409569]

Robinson EB, Lichtenstein P, Anckarsäter H, Happé F, Ronald A (2013). Examining and interpreting the female protective effect against autistic behavior. Proc Natl Acad Sci 110:5258-5262. [PubMed: 23431162]

Rossin EJ, Lage K, Raychaudhuri S, Xavier RJ, Tatar D, Benita Y, et al. (2011). Proteins encoded in genomic regions associated with immune-mediated disease physically interact and suggest underlying biology. PLoS Genet 7: e1001273. [PubMed: 21249183]

Salzano FM, Sans M (2014). Interethnic admixture and the evolution of LatinAmerican populations. Genet Mol Biol 37:151-170. [PubMed: 24764751]

Santos IS, Barros AJ, Matijasevich A, Zanini R, Chrestani Cesar MA, Camargo-Figuera FA, et al. (2014). Cohort profile update: 2004 Pelotas (Brazil) Birth Cohort Study. Body composition, mental health and genetic assessment at the 6 years follow-up. Int J Epidemiol 43:1437-1437f. [PubMed: 25063002]

Schaakxs R, Verhoeven JE, Oude Voshaar RC, Comijs HC, Penninx BW (2015). Leukocyte telomere length and late-life depression. Am J Geriatr Psychiatry 23:423-432. [PubMed: 25028345]

Schalkwyk LC, Meaburn EL, Smith R, Dempster EL, Jeffries AR, Davies MN, et al. (2010). Allelic skewing of DNA methylation is widespread across the genome. Am J Hum Genet 86:196-212. [PubMed: 20159110]

Schizophrenia Psychiatric Genome-Wide Association Study (GWAS) Consortium (2011). Genomewide association study identifies five new schizophrenia loci. Nat Genet 43:969-976. [PubMed: 21926974]

Schizophrenia Working Group of the Psychiatric Genomics Consortium (2014). Biological insights from 108 schizophrenia-associated genetic loci. Nature 511:421-427. [PubMed: 25056061]

Schmidt M, Pedersen L, Sorensen HT (2014). The Danish Civil Registration System as a tool in epidemiology. Eur J Epidemiol 29:541-549. [PubMed: 24965263] 
Schneider M, Debbané M, Bassett A, Chow E, Fung W, van den Bree M, et al. (2014). Psychiatric disorders from childhood to adulthood in 22q11.2 deletion syndrome: results from the International Consortium on Brain and Behavior in 22q11.2 Deletion Syndrome. Am J Psychiatry 171:627-639. [PubMed: 24577245]

Schork AJ, Thompson WK, Pham P, Torkamani A, Roddey JC, Sullivan PF, et al. (2013). All SNPs are not created equal: genome-wide association studies reveal a consistent pattern of enrichment among functionally annotated SNPs. PLoS Genet 9:e1003449. [PubMed: 23637621]

Segura-Wang M, Raventós H, Escamilla M, Barrantes R (2010). Assessment of genetic ancestry and population substructure in Costa Rica by analysis of individuals with a familial history of mental disorder. Ann Hum Genet 74:516-524. [PubMed: 20946256]

Sequeira A, Gwadry FG, Ffrench-Mullen JM, Canetti L, Gingras Y, Casero RA Jr, et al. (2006). Implication of SSAT by gene expression and genetic variation in suicide and major depression. Arch Gen Psychiatry 63:35-48. [PubMed: 16389195]

Sequeira PA, Martin MV, Vawter MP (2012). The first decade and beyond of transcriptional profiling in schizophrenia. Neurobiol Dis 45:23-36. [PubMed: 21396449]

Shalev I, Moffitt TE, Braithwaite AW, Danese A, Fleming NI, Goldman-Mellor S, et al. (2014). Internalizing disorders and leukocyte telomere erosion: a prospective study of depression, generalized anxiety disorder and post-traumatic stress disorder. Mol Psychiatry 19:1163-1170. [PubMed: 24419039]

Shinawi M, Liu P, Kang SH, Shen J, Belmont JW, Scott DA, et al. (2010). Recurrent reciprocal 16 p11.2 rearrangements associated with global developmental delay, behavioural problems, dysmorphism, epilepsy, and abnormal head size. J Med Genet 47:332-341. [PubMed: 19914906]

Simpson AJG, Reinach FC, Arruda P, Abreu FA, Acencio M, Alvarenga R, et al. (2000). The genome sequence of the plant pathogen Xylella fastidiosa. Nature 406:151-157. [PubMed: 10910347]

Sokolowska E, Hovatta I (2013). Anxiety genetics - findings from cross-species genome-wide approaches. Biol Mood Anxiety Disord 3:9. [PubMed: 23659354]

Song J, Bergen SE, Kuja-Halkola R, Larsson H, Landen M, Lichtenstein P (2015). Bipolar disorder and its relation to major psychiatric disorders: a family-based study in the Swedish population. Bipolar Disord 17:184-193. [PubMed: 25118125]

Stefánsson H, Meyer-Lindenberg A, Steinberg S, Magnusdottir B, Morgen K, Arnarsdottir S, et al. (2014). CNVs conferring risk of autism or schizophrenia affect cognition in controls. Nature 505:361-366. [PubMed: 24352232]

Stein JL, Medland SE, Vasquez AA, Hibar DP, Senstad RE, Winkler AM, et al. (2012). Identification of common variants associated with human hippocampal and intracranial volumes. Nat Genet 44:552-561. [PubMed: 22504417]

Stein JL, de la Torre-Ubieta L, Tian Y, Parikshak NN, Hernández IA, Marchetto MC, et al. (2014). A quantitative framework to evaluate modeling of cortical development by neural stem cells. Neuron 83:69-86. [PubMed: 24991955]

Straub RE, Lipska BK, Egan MF, Goldberg TE, Callicott JH, Mayhew MB, et al. (2007). Allelic variation in GAD1 (GAD67) is associated with schizophrenia and influences cortical function and gene expression. Mol Psychiatry 12:854-869. [PubMed: 17767149]

Strömgren E (1982). Erik Strongren In: Shepherd M, editor. Psychiatrists on psychiatry. Cambridge: University Press pp. 152-158.

Suarez-Kurtz G, Paula DP, Struchiner CJ (2014). Pharmacogenomic implications of population admixture: Brazil as a model case. Pharmacogenomics 15:209-219. [PubMed: 24444410]

Suez J, Korem T, Zeevi D, Zilberman-Schapira G, Thaiss CA, Maza O, et al. (2014). Artificial sweeteners induce glucose intolerance by altering the gut microbiota. Nature 514:181-186. [PubMed: 25231862]

Szatkiewicz JP, O’Dushlaine C, Chen G, Chambert K, Moran JL, Neale BM, et al. (2014). Copy number variation in schizophrenia in Sweden. Mol Psychiatry 19:762-773. [PubMed: 24776740]

Thorgeirsson TE, Geller F, Sulem P, Rafnar T, Wiste A, Magnusson KP, et al. (2008). A variant associated with nicotine dependence, lung cancer and peripheral arterial disease. Nature 452:638-642. [PubMed: 18385739] 
Trapnell C, Williams BA, Pertea G, Morazavi A, Kwan G, van Baren MJ, et al. (2010). Transcript assembly and quantification by RNA-Seq reveals unannotated transcripts and isoform switching during cell differentiation. Nat Biotechnol 28:511-515. [PubMed: 20436464]

Trivedi MH, Rush AJ, Wisniewski SR, Nierenberg AA, Warden D, Ritz L, et al. (2006). Evaluation of outcomes with citalopram for depression using measurement-based care in STAR*D: implications for clinical practice. Am J Psychiatry 163:28-40. [PubMed: 16390886]

Tsang SY, Zhong S, Mei L, Chen J, Ng SK, Pun FW, et al. (2013). Social cognitive role of schizophrenia candidate gene GABRB2. Plos One 8:e62322. [PubMed: 23638040]

Tye KM, Prakash R, Kim S, Fenno LE, Grosenick L, Zarabi H, et al. (2011). Amygdala circuitry mediating reversible and bidirectional control of anxiety. Nature 471:358-362. [PubMed: 21389985]

Uher R, Perroud N, Ng MY, Hauser J, Henigsberg N, Maier W, et al. (2010). Genome-wide pharmacogenetics of antidepressant response in the GENDEP project. Am J Psychiatry 167:555564. [PubMed: 20360315]

Uhl GR, Drgon T, Liu Q-R, Johnson C, Walther D, Komiyama T, et al. (2008). Genome-wide association for methamphetamine dependence: convergent results from 2 samples. Arch Gen Psychiatry 65:345-355. [PubMed: 18316681]

van der Sluis S, Posthuma D, Dolan CV (2013). TATES: efficient multivariate genotype-phenotype analysis for genome-wide association studies. PLoS Genet 9:e1003235. [PubMed: 23359524]

Velez-Pardo C, Rojas W, Jimenez-Del-Rio M, Bedoya G (2015). Distribution of APOE polymorphism in the 'paisa' population from northwest Colombia (Antioquia). Ann Hum Biol 42:195-198. [PubMed: 25026367]

Verhoeven JE, Révész D, Epel ES, Lin J, Wolkowitz OM, Penninx BW (2014a). Major depressive disorder and accelerated cellular aging: results from a large psychiatric cohort study. Mol Psychiatry 19:895-901. [PubMed: 24217256]

Verhoeven JE, Révész D, Wolkowitz OM, Penninx BW (2014b). Cellular aging in depression: permanent imprint or reversible process?: an overview of the current evidence, mechanistic pathways, and targets for interventions. Bioessays 36:968-978. [PubMed: 25143317]

Verhoeven JE, Revezs D, van Oppen P, Wolkowitz O, Penninx BW (2015). Anxiety disorders and cellular aging. Br J Psychiatry 206:371-378. [PubMed: 25657360]

Voineagu I, Wang X, Johnston P, Lowe JK, Tian Y, Horvath S, et al. (2011). Transcriptomic analysis of autistic brain reveals convergent molecular pathology. Nature 474:380-384. [PubMed: 21614001]

Vos M, Esposito G, Edirisinghe JN, Vilain S, Haddad DM, Slabbaert JR, et al. (2012). Vitamin K2 is a mitochondrial electron carrier that rescues pink1 deficiency. Science 336:1306-1310. [PubMed: 22582012]

Wang K, Zhang H, Bloss CS, Duvvuri V, Kaye W, Schork NJ, et al. (2011). A genome-wide association study on common SNPs and rare CNVs in anorexia nervosa. Mol Psychiatry 16:949959. [PubMed: 21079607]

Weaver ICG, Cervoni N, Champagne FA, D’Alessio AC, Sharma S, Seckl JR, et al. (2004). Epigenetic programming by maternal behaviour. Nat Neurosci 7: 847-854. [PubMed: 15220929]

Wietek J, Wiegert S, Adeishvili N, Schneider F, Watanabe H, Tsunoda SP, et al. (2014). Conversion of channelrhodopsin into a light-gated chloride channel. Science 344:409-412. [PubMed: 24674867]

Wolkowitz OM, Mellon SH, Epel ES, Lin J, Dhabhar FS, Su Y, et al. (2011). Leukocyte telomere length in major depression: correlations with chronicity, inflammation and oxidative stress preliminary findings. PLoS One 6:e17837. [PubMed: 21448457]

Yang J, Lee SH, Goddard ME, Visscher PM (2011). GCTA: a tool for genome-wide complex trait analysis. Am J Hum Genet 88:76-82. [PubMed: 21167468]

Yang WY, Novembre J, Eskin E, Halperin E (2012). A model-based approach for analysis of spatial structure in genetic data. Nat Genet 44:725-731. [PubMed: 22610118]

Yang J, Zaitlen NA, Goddard ME, Visscher PM, Price AL (2014). Advantages and pitfalls in the application of mixed-model association methods. Nat Genet 46:100-106. [PubMed: 24473328] 
Yehuda R, Daskalakis NP, Desarnaud F, Makotkine I, Lehrner AL, Koch E, et al. (2013). Epigenetic biomarkers as predictors and correlates of symptom improvement following psychotherapy in combat veterans with PTSD. Front Psychiatry 4:118. [PubMed: 24098286]

Zhang JP, Lencz T, Malhotra AK (2010). $\mathrm{D}_{2}$ receptor genetic variation and clinical response to antipsychotic drug treatment: a meta-analysis. Am J Psychiatry 167:763-772. [PubMed: 20194480]

Zhao C, Xu Z, Chen J, Yu Z, Tong KL, Lo WS, et al. (2006). Two isoforms of GABAA receptor beta2 subunit with different electrophysiological properties: Differential expression and genotypical correlations in schizophrenia. Mol Psychiatry 11:1092-1105. [PubMed: 16983389]

Zhao C, Xu Z, Wang F, Chen J, Ng SK, Wong PW, et al. (2009). Alternative-splicing in the exon-10 region of GABAA receptor beta(2) subunit gene: relationships between novel isoforms and psychotic disorders. Plos One 4: e6977. [PubMed: 19763268]

Zhao C, Wang F, Pun FW, Mei L, Ren L, Yu Z, et al. (2012). Epigenetic regulation on GABRB2 isoforms expression: developmental variations and disruptions in psychotic disorders. Schizophr Res 134:260-266. [PubMed: 22206711]

Zhou K, Dempfle A, Arcos Burgos M, Bakker SC, Banaschewski T, Biederman J, et al. (2008). Meta analysis of genome wide linkage scans of attention deficit hyperactivity disorder. Am J Med Genet Part B Neuropsychiatr Genet 147:1392-1398.

Zhou QG, Hu Y, Wu DL, Zhu LJ, Chen C, Jin X, et al. (2011). Hippocampal telomerase is involved in the modulation of depressive behaviors. J Neurosci 31:12258-12269. [PubMed: 21865469]

Zisook S, Ganadjian K, Moutier C, Prather R, Rao S (2008). Sequenced Treatment Alternatives to Relieve Depression (STAR*D): lessons learned. J Clin Psychiatry 69:1184-1185. [PubMed: 18687018]

Zuk O, Schaffner SF, Samocha K, Do R, Hechter E, Kathiresan S, et al. (2014). Searching for missing heritability: designing rare variant association studies. Proc Natl Acad Sci USA 111:E455-E464. [PubMed: 24443550] 